University of Louisville

ThinkIR: The University of Louisville's Institutional Repository

Electronic Theses and Dissertations

$5-2013$

\title{
Molecular bases of inherited bone marrow failures : Shwachman- Diamond syndrome and Diamond Blackfan anemia.
}

Adrianna Lee Henson 1983-

University of Louisville

Follow this and additional works at: https://ir.library.louisville.edu/etd

\section{Recommended Citation}

Henson, Adrianna Lee 1983-, "Molecular bases of inherited bone marrow failures : Shwachman-Diamond syndrome and Diamond Blackfan anemia." (2013). Electronic Theses and Dissertations. Paper 608. https://doi.org/10.18297/etd/608

This Doctoral Dissertation is brought to you for free and open access by ThinkIR: The University of Louisville's Institutional Repository. It has been accepted for inclusion in Electronic Theses and Dissertations by an authorized administrator of ThinkIR: The University of Louisville's Institutional Repository. This title appears here courtesy of the author, who has retained all other copyrights. For more information, please contact thinkir@louisville.edu. 
MOLECULAR BASES OF INHERITED BONE MARROW FAILURES: SHWACHMAN-DIAMOND SYNDROME AND DIAMOND BLACKFAN ANEMIA

\title{
By
}

Adrianna Lee Henson

B.S., Wake Forest University, 2005

\begin{abstract}
A Dissertation
Submitted to the Faculty of the School of Medicine of the University of Louisville In Partial Fulfillment of the Requirements For the Degree of
\end{abstract}

Doctor of Philosophy

Department of Biochemistry and Molecular Biology University of Louisville

Louisville, KY

May 2013 

MOLECULAR BASES OF INHERITED BONE MARROW FAILURES:

SHWACHMAN-DIAMOND SYNDROME AND DIAMOND BLACKFAN ANEMIA

By

Adrianna Lee Henson

B.S., Wake Forest University, 2005

A Dissertation Approved on

August 15, 2012

By the following Dissertation Committee:

Dissertation Director: Dr. Steve Ellis

Dr. William Dean

Dr. Chuan $\mathrm{Hu}$

Dr. Robert Mitchell

Dr. Brian Wattenberg 


\section{DEDICATION}

This dissertation is dedicated to my family.

Without the blessing of their love and support, I would never have dared to dream. 


\section{ACKNOWLEDGEMENTS}

First, I would like to first thank my committee for their encouragement, flexibility and scientific insight. Dr. Hu, thank you for always being a cheerful face when I passed by and for helping me to see things in a positive light. I found you to be very encouraging to know that you took a genuine interest in me as well as my work. Dr. Dean, thank you for pushing me to ask more questions and perform the most thorough scientific investigations possible. Thank you also, for providing a role model for scientific teaching. You were never too busy to help a student in your lab and left your office door open to anyone with questions. Dr. Mitchell, thank you for probing with questions I would never have thought to ask otherwise. You were always looking for alternative strategies or mechanisms of scientific inquiry. Binks, thank you for your support not only during my graduate years, but also through the MD/PhD program. You have worked tirelessly to provide many opportunities for us to develop the program to our individual needs and to use our strengths to enrich the program also. Also, thank you for demonstrating on a daily basis how a passion for science adds zest to life.

To my mentor Dr. Ellis, this would not be possible without you. When I asked to work with you many years ago, I knew you would challenge me. I had no idea then what would later ensue. You have provided me with rigorous scientific training not only in benchwork, but also the ins and outs of daily life as a clinician scientist. Your collaborations with numerous people in the bone marrow 
failure field have allowed me to interact with all levels and specialties of researchers. I am grateful for the unique opportunity I had to observe young scientist starting their own labs and balancing clinical and scientific duties.

We may not always agree on everything, but I know you did your very best to teach me how to be passionate about my work. Your expectations of efficiency were reasonable because you have led through example. You also showed me the joy it brings to teach others: students of all ages, professors, clinicians, lay persons in your church: anyone, and everyone can benefit from the knowledge of science. The more opportunities you are presented, the more important it is for you to share with others. I am one of the only graduate students I know, at any institution or in any field, that can say their boss took the time to painstaking teach them how to do experiments and you taught me several. You also taught me to be humble. There will always be other people that are just as capable, so it's important to work efficiently and consistently to survive in science. I hope to one day embody the spirit of my work with the gusto and bravery with which you ask scientific questions.

Thank you to all of the brilliant people l've had the privilege of working with both in Louisville and abroad. At some points it seemed that I had a million things going on at once, but I appreciate the opportunity to work on many interesting topics. Nothing in this document was done alone. I was continually blessed to work with such helpful people. A special thank you must go to Dr. Sharon Singh and her family who hosted me on a trip to FIMR this past fall. Her productivity as 
a scientist, skill as a physician, and compassion as a mother are a wonderful example.

Thank you to all my fellow lab members. So many people contributed to the work contained in this dissertation that it would be difficult to mention them all, but l'd like to give a special thank you to Dr. Elisa Pavesi. She taught me so much during my first semester in graduate school and I would have been lost without her camaraderie. Thank you also to Renate Meier and the Clark lab for have a never ending supply of helpful information and to Dr. Pascale Alard who took countless hours to patiently teach me about FACS as a student from another department with no background in the topic. I could not ask for anyone to be more patient with me than you were. Thank you also to the biochemistry department and the MD/PhD program for all of your support in so many ways. I would be remiss to fail to mention the special place in my heart that Julian will always have. He always watched out for me when I was in lab late at night, and reminded me to have patience with myself and also with my mentor. I have learned a lot from him over the years both about gardening and about life. 


\section{ABSTRACT \\ MOLECULAR BASES OF INHERITED BONE MARROW FAILURES: SHWACHMAN-DIAMOND SYNDROME AND DIAMOND BLACKFAN ANEMIA}

\section{Adrianna Lee Henson}

August 15, 2012

Inherited Bone Marrow Failure syndromes (IBMFS) are a heterogeneous class of diseases that converge on very few subjects. They are unified by a block in maturation of one or multiple blood lineages, are genetically inherited, and have an increased incidence of cancers and myelodisplastic syndromes. Several IBMFSs have been linked to defects in ribosome biogenesis. Diamond Blackfan anemia (DBA) which presents with a macrocytic anemia has 10 known affected genes all of which encode structural proteins of the large or small ribosomal subunit. In contrast, there is only a single gene known to be involved in the pathogenesis of Shwachman Diamond Syndrome (SDS) which usually presents as exocrine pancreatic insufficiency along with neutropenia.

Investigation of DBA pathogenesis lead to the knowledge that deficiency of certain ribosomal proteins leads to a specific defect in ribosomal pre-RNA processing. In this work, we have identified a new DBA gene, $R P L 31$, by isolating mononuclear cells from the patient's blood and subsequently harvesting total RNA used for Northern blotting to identify delays in pre-rRNA processing in both this patient as well as those found in a patient with other large subunit DBA 
mutations. Additionally, we have created a human cell model of SDS that has allowed us to explore changes in respiration originally shown in yeast models. To this end, we have identified increases in reactive oxygen species, changes in oxygen consumption, mitochondrial membrane potential, and cell cycle delay, all linked to depletion of SBDS protein. 


\section{TABLE OF CONTENTS}

PAGE

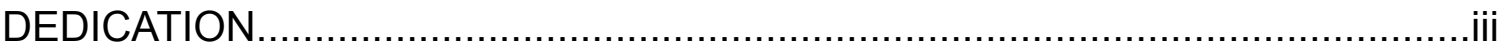

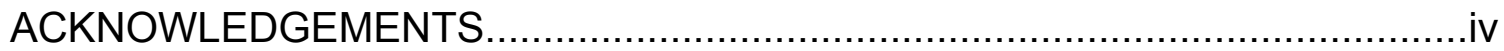

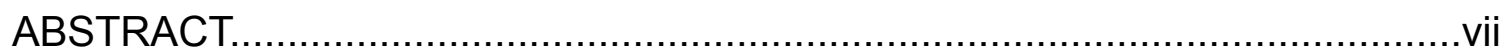

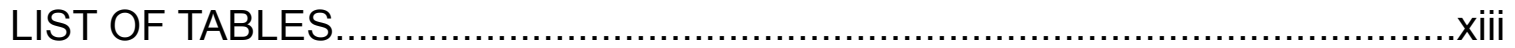

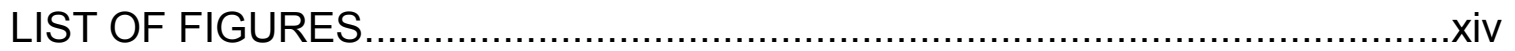

\section{CHAPTER}

I. RIBOSOME DYSFUNCTION AND MARROW FAILURE

The Mammalian Ribosome: Structure and Function.................................1

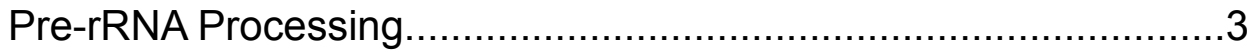

Synthesis of Ribosomal Species............................................

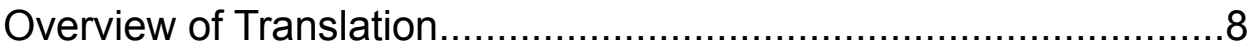

Peptide Bond Formation........................................................ 10

Bone Marrow Failure syndromes and other Ribosomopathies................11

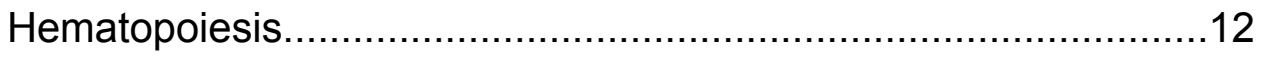

Diamond Blackfan anemia: Pathophysiology.............................14

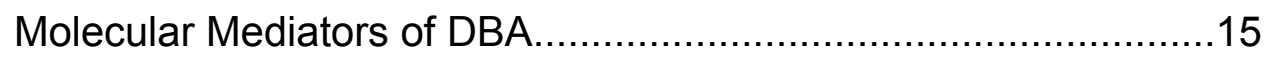

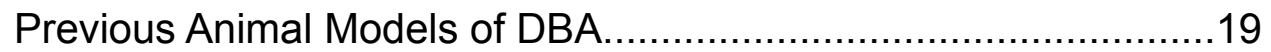

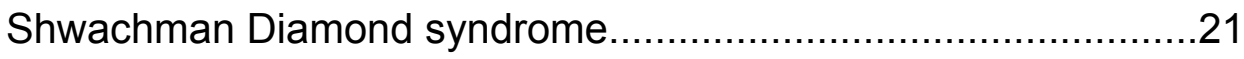

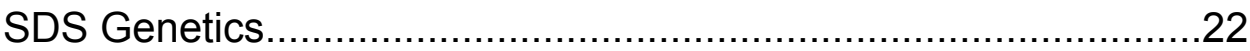

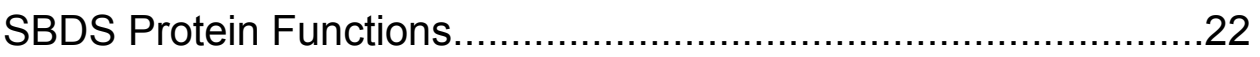

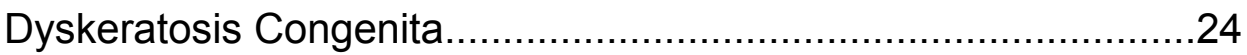




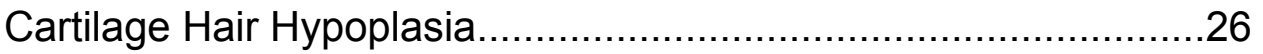

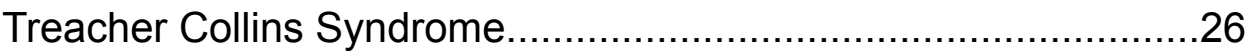

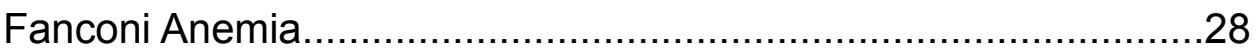

Severe Congenital Neutropenia...............................................30

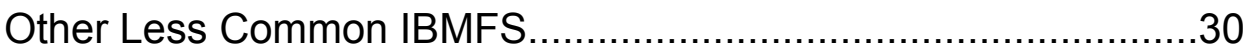

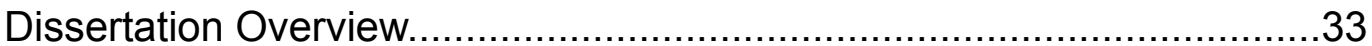

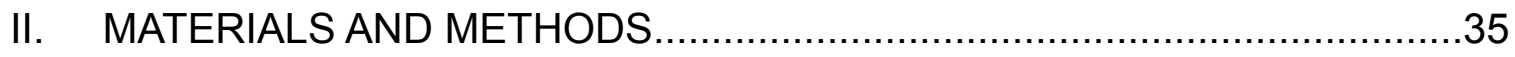

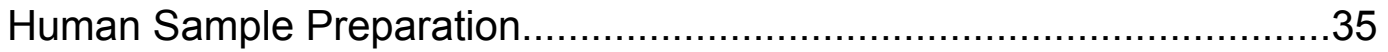

Buffy Coat Preparation of Mononuclear Cells and Stimulation

with ConA

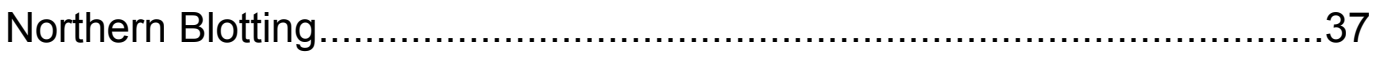

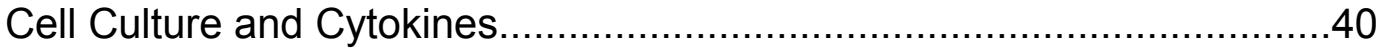

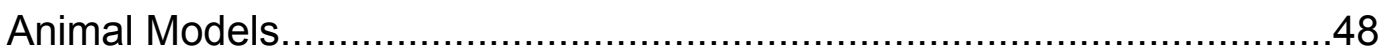

Cell Viability and Rescue with NAC...................................................

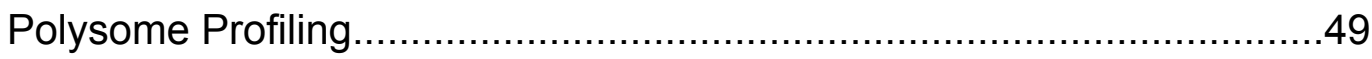

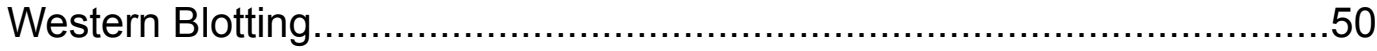

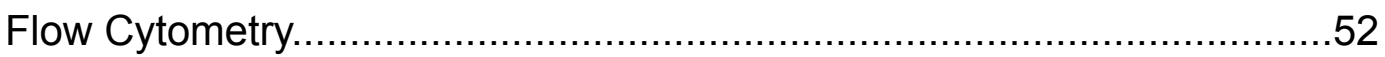

Reactive Oxygen Species Generation..................................................55

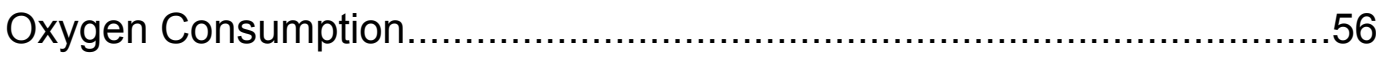

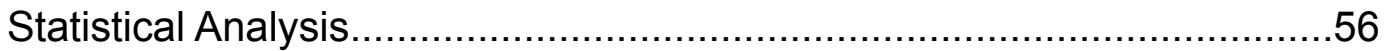

III. THE VITAL ROLE OF PRE-rRNA PROCESSING IN DBA

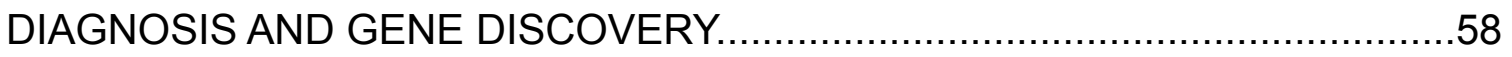

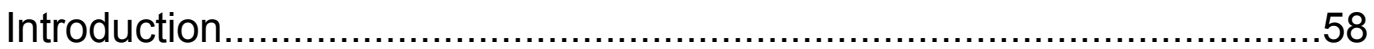

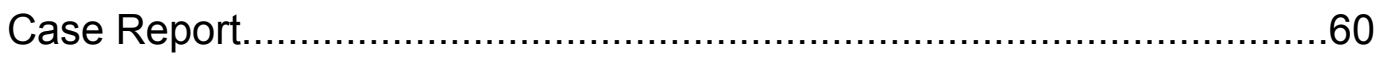


Results. .63

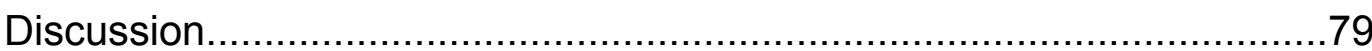

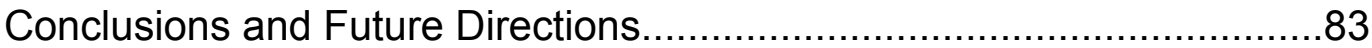

\section{MITOCHONDRIAL DYSFUNCTION IN A HUMAN CELL LINE} MODEL OF SHWACHMAN DIAMOND SYNDROME ..............................84

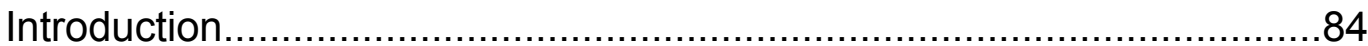

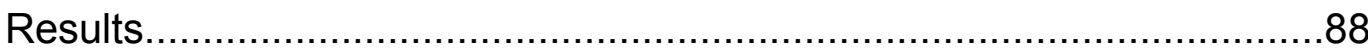

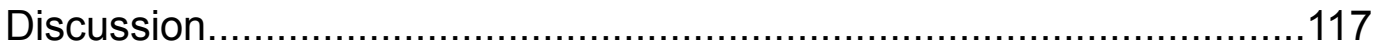

Conclusions and Future Directions...................................................124

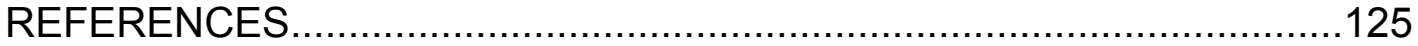

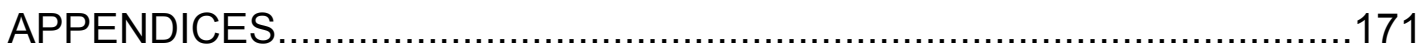

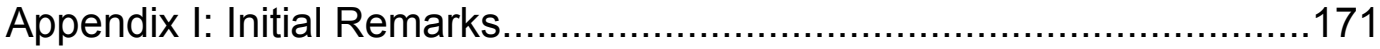

Section 1: Mouse Embryonic Stem Cell Model....................................172

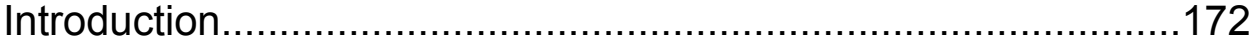

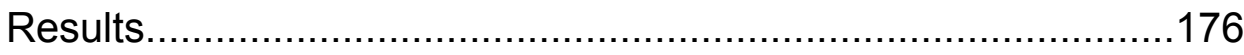

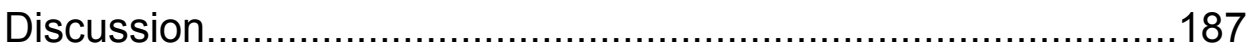

Conclusions and Future Directions........................................188

Section 2: Inducible Rps19 Depletion Mouse Model.............................189

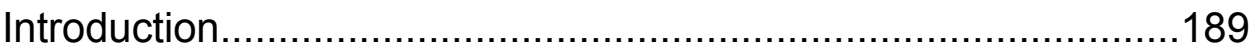

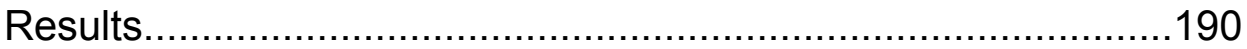

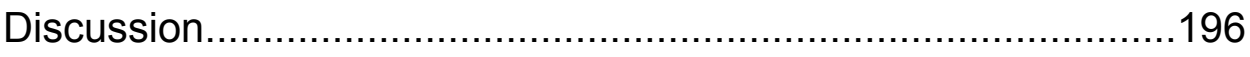

Conclusions and Future Directions........................................198 


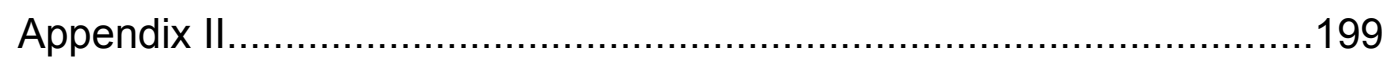

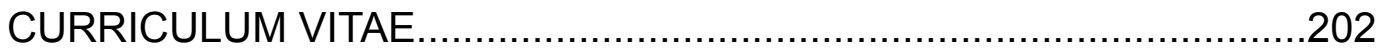




\section{LIST OF TABLES}

\section{TABLE}

PAGE

1. Northern blot probes used for the identification of pre-rRNA

processing and mature rRNA species formation..........................................39

2. Mitochondrial proteins analyzed using immunoblotting.............................109 


\section{LIST OF FIGURES}

\section{FIGURE}

1. Pre-rRNA is derived from a polycistronic sequence and

PAGE multiple processing steps.

2. The $5 \mathrm{~S}$ rRNA subcomplex as a critical mediator of ribosome stress signaling to $p 53$ activation.

3. Insertion of the ROSAFARY gene trap vector between exon 2 and exon 3 of Rps19 gene created the S17 gene trap cell line

4. Location of gene trap insertions in mES cell lines used

5. Pre-rRNA processing defect associated with haploinsufficiency for RPL31

6. Northern blot of RNA isolated from the index proband, unaffected parents, and a healthy control reveals specific processing defect in pre-rRNA.

7. Evidence of a common pre-rRNA processing signature associated with haploinsufficiency for large subunit ribosomal proteins

8. Northern Blots of DBA patient samples reveal a unique processing signature in a patient where RPL5 is mutated

9. Polysome profiles of TF-1 samples depleted of RPS19 show an increase in the free pool of 5S rRNA

10. Free $5 S$ rRNA is increased in mouse embryonic stem cells heterozygous for Rps19.

11. Free $5 S$ rRNA is unchanged in mouse embryonic stem cells heterozygous for $R p / 5$.

12. Cells heterozygous for either Rps19 or $R p / 5$ show an increase in p53 expression relative to controls. 
13. Por1 expression is increased in $\triangle \mathrm{SDO} 1$ yeast strain

14. Steady-state amounts of SBDS protein and mRNA in TF-1 clones.

15. Cells depleted of SBDS have a reduced growth rate relative to scrambled controls.

16. Polysome profiles from TF-1 cells depleted of SBDS and a scrambled control

17. Distribution of elF6 in sucrose gradients derived from TF-1 cells depleted of SBDS and scrambled controls

18. Analysis of cell cycle progression in cells depleted of SBDS and scrambled controls.

19. Analysis of erythroid and granulocyte differentiation of TF-1 cells depleted of SBDS or scrambled controls.

20. Oxygen consumption by TF-1 cells depleted of SBDS compared with scrambled controls

21. Analysis of mitochondrial membrane potential in cells depleted of SBDS and scrambled controls. 106

22. Multiple mitochondrial proteins change expression levels with SBDS knockdown.

23. Analysis of VDAC1 expression in TF-1 cells depleted of SBDS compared with scrambled controls.

24. Analysis of ROS levels in TF-1 cells depleted of SBDS

25. Complex effects of $\mathrm{N}$-acetyl-cysteine on growth and viability of TF-1 cell cultures.

S1. Embryonic stem cell culture recapitulates hematopoiesis in mouse models.

S2. Protein knockdown of Rps19 in mES cells evidenced by Western blotting

S3. Protein knockdown of Rpl5 in mES gene trap samples 179 
S4. mES samples display processing defect in pre-rRNA specific to haploinsufficiency in Rps19.

S5. mES samples with haploinsufficiency for $R p / 5$ show a decrease in polysome size

S6. mES polysome profiles show small subunit defect with Rps19 genetrap 185

S7. Pre-rRNA processing defects associate with inducible Rps19 shRNA expressing mouse model.

S8. Polysome profile defects specific to small ribosomal subunit in inducible Rps19 shRNA mouse model....

S9. Peak weights of liver rRNA species separated on sucrose gradients and identified by polysome profiling. 


\section{CHAPTER I}

\section{RIBOSOME DYSFUNCTION AND MARROW FAILURE}

\section{The Mammalian Ribosome: Structure and Function}

The ribosome is responsible for translating the coded messages of mRNA and catalyzing peptide bond formation between individual amino acids to generate proteins. The ribosome thus functions as an enzyme, or ribozyme due to its catalytic activity being embodied in its RNA rather than protein components (Cech, 2000). Recent work on the crystal structure for the mammalian ribosome has provided insight into the spatial arrangement of proteins and RNAs forming this remarkable complex (Ben-Shem et al., 2010). The mammalian ribosome is composed of four distinct ribosomal RNAs (rRNAs), and 80 ribosomal proteins (RPs). Ribosomal proteins are named using the nomenclature of RPL for proteins of the large ribosomal subunit and RPS for the small ribosomal subunit. In addition to its structural components, ribosome synthesis requires more than 200 essential non-ribosomal trans-acting factors including both accessory proteins and small nucleolar ribonucleoprotein particles (snoRNPs) (Jackson et al., 2010). Large structures like ribosomes are often spoken of in terms of Svedberg units based on their rate of migration in a centrifugal field, such as 40S, for the small ribosomal subunit. During protein synthesis, the $40 \mathrm{~S}$ subunit 
binds to mRNA and scans down its length until it encounters an AUG start codon. This complex, known as the $40 \mathrm{~S}$ initiation complex, requires a number of initiation factors and GTP to bind and once formed awaits the joining of a 605 subunit to form the $80 \mathrm{~S}$ initiation complex. Formation of the $80 \mathrm{~S}$ initiation complex requires energy, the recruitment of additional initiation factors, and the release of others. Once formed, the $80 \mathrm{~S}$ initiation complex is ready to enter the elongation phase of protein synthesis as the ribosome translates down the mRNA.

The production of ribosomal subunits is exquisitely regulated such that equal amounts of $40 S$ and 605 subunits are produced in response to a number of physiologic, developmental, and environmental cues in amounts that are proportional to the needs of the cell (reviewed in (Warner, 1990)). This process is highly complex and occurs in a specialized organelle called the nucleolus, which is found within the nucleus. Ribosome synthesis involves the coordinate activities of RNA polymerase I and III producing RNA components of the ribosome and RNA polymerase II producing transcripts for ribosomal proteins. Ribosomal protein transcripts are translated in the cytoplasm and proteins imported into the nucleus and subsequently travel to the nucleolus where the rRNAs are being transcribed. Ribosome assembly occurs co-transcriptionally as ribosomal proteins load onto nascent RNA polymerase I transcripts in forming pre-ribosomal subunits. These pre-subunits continue to mature as additional ribosomal proteins are incorporated, $5 \mathrm{~S}$ rRNA produced by RNA polymerase III is also incorporated into maturing $60 \mathrm{~S}$ subunits. Ribosome biogenesis also 
requires post transcriptional and post translational modifications of ribosomal RNA and protein, respectively. Finally, additional factors are involved in subunit transport from the nucleus to the cytoplasm (Zemp and Kutay, 2007).

\section{Pre-rRNA processing}

Three of the four mature rRNAs are synthesized from a large polycistronic 45S pre-rRNA transcript synthesized by RNA polymerase I. This organization assures the equimolar production $18 \mathrm{~S}, 5.8 \mathrm{~S}$ and $28 \mathrm{~S}$ RNA components of the ribosome. Only the $5 \mathrm{~S}$ rRNA, transcribed by RNA polymerase III, is not found in this organizational unit. In yeast, the rDNA unit includes coding regions for both $5 S$ rRNA and the 45S RNA. This coding unit is repeated from 30 to 500 times in the genome and this spatial organization may allow the coordination of RNA polymerase I and III activities in producing the four rRNAs. In mammalian cells, the 5S rDNA is not physically linked to the remaining rDNA repeat units. The rDNA repeats are clustered at various sub-telomeric regions on different chromosomes. The basis for locating $5 \mathrm{~S}$ rDNA independently of the other rDNA repeat units in many higher organisms, including humans, is unknown (Ciganda and Williams, 2011).

In addition to the mature $18 \mathrm{~S}$ rRNA of the $40 \mathrm{~S}$ subunit and the $5.8 \mathrm{~S}$ and $28 \mathrm{~S}$ rRNAs of the $60 \mathrm{~S}$ subunit, the $45 \mathrm{~S}$ pre-rRNA contains external flanking sequences (ETS: external transcribed sequences) and two internal transcribed sequences (ITS1 and ITS2). Thus, to liberate the mature rRNA species the 45S pre-rRNA must undergo a series of endo- and exonucleolytic processing steps 
which occur concurrently with the assembly of ribosomal proteins onto nascent transcripts. Other processing events occur co-transcriptionally to the $45 \mathrm{~S}$ prerRNA including methylation and pseudouridinylation (Fromont-Racine et al., 2003). The current view of the numerous steps involved in the maturation of mammalian 45S pre-RNA are outlined in a simplified version in Fig. 1. The vast majority of these processing steps occur in the nucleolus although some later steps in maturation occur in the cytoplasm (Flygare et al., 2007). Limiting the amount of many of the factors required for ribosome synthesis have been shown to cause delays in maturation of pre-rRNA as evidenced by the accumulation of distinct pre-rRNA intermediates. For example, when one copy of RPS19 is mutated in patients with an inherited bone marrow failure syndrome (IBMFS), Diamond Blackfan anemia (DBA), there is a delay in the maturation of the $3^{\prime}$ end of $18 \mathrm{~S}$ rRNA which can be visualized by Northern blotting as an increase in $21 \mathrm{~S}$ pre-rRNA (Flygare et al., 2007). This strategy of employing Northern blot analysis to identify specific defects during the process of ribosome synthesis is used throughout the first part of this dissertation. This will be discussed in more detail in subsequent chapters and Northern blot oligonucleotides used in this dissertation to identify processing defects in pre-rRNA resulting from mutations in genes encoding ribosomal proteins are shown in Table 1 of Chapter 2. 


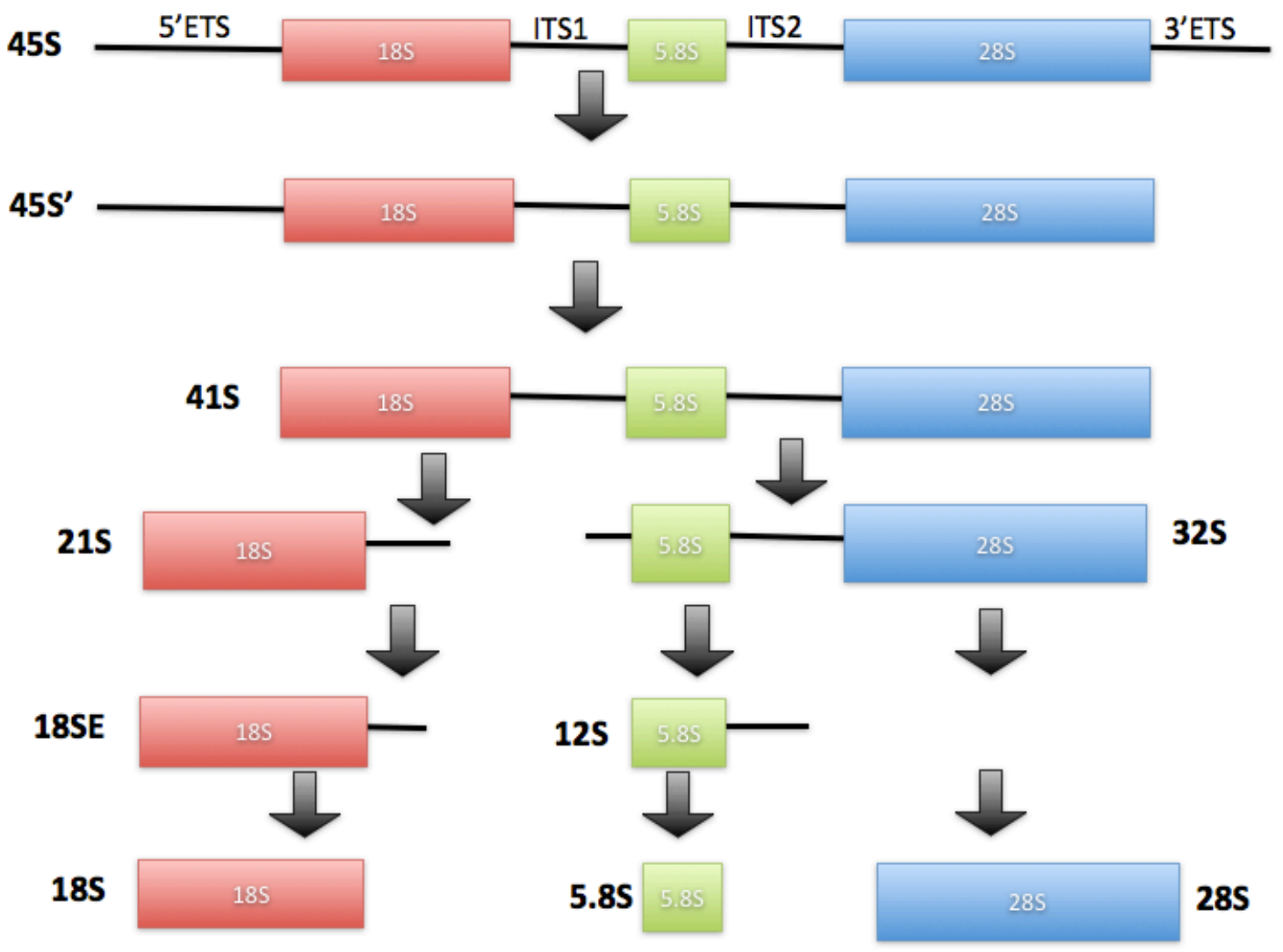

Fig 1: Mature rRNAs are liberated from a large polycistronic transcript by a series of endo and exonucleolytic cleavage steps.

The $45 \mathrm{~S}$ pre-rRNA transcribed by RNA polymerase I is shown at the top of the figure. This transcript undergoes multiple exonuclease and endonuclease processing steps to form mature $18 \mathrm{~S}, 5.8 \mathrm{~S}$, and $28 \mathrm{~S}$ rRNAs. The pathway shown here is the major pathway found in mononuclear cells derived from human blood and in the TF-1 human erythroleukemia cell line. 


\section{Synthesis of Ribosomal Subunits}

While pre-rRNA processing is a way of tracking different steps in ribosome synthesis, these processing events are only a small part of the overall process of ribosome synthesis that begins with nucleolar transcription by RNA polymerase I and ends with functional ribosomal subunits in the cytoplasm. The "Christmas tree" appearance of pre-rRNA transcription was described decades ago (Miller and Beatty, 1969). These nascent transcripts begin at rDNA promoters and get longer the further they extend from the initiation site. The high transcription rates of rDNA in rapidly dividing cells give rise to numerous pre-rRNAs being transcribed simultaneously giving the characteristic Christmas tree appearance. More detailed Miller spreads of rDNA transcription units in eukaryotic cells have revealed that these nascent pre-rRNA transcripts begin to ball up as ribosomal proteins and various trans-acting factors assemble co-transcriptionally on the 45S pre-rRNA (Miller and Beatty, 1969).

The earliest complex in the process of ribosome synthesis that can be biochemically identified is the 905 pre-ribosomal particle. This complex includes the small subunit processome (SSU) which gathers on the 18S rRNA domain at the 5 '-end of the $45 S$ pre-rRNA. The SSU includes the U3 snoRNP which is involved in promoting endonucleolytic cleavages in the 5'-ETS and ITS1 and so plays a critical role in the maturation of the $40 \mathrm{~S}$ ribosomal subunit (Dragon et al., 2002). Although numerous ribosomal proteins and factors required for the assembly of the $40 S$ subunit are found in the 905 species, no large subunit 
ribosomal proteins or 605 trans-acting factors are found in this early complex (Gavin et al., 2002; Grandi et al., 2002).

After the $90 \mathrm{~S}$ species goes through the processing steps in ITS1, the pre-40S subunits separate from the pre-60S subunit to begin further independent steps in subunit maturation. Pre-40S subunits containing an immature $20 \mathrm{~S}$ prerRNA recruit additional proteins as maturation continues, and pre-40S subunits are transported out of the nucleus. For example Tsr1 is necessary for processing of $20 \mathrm{~S}$ pre-rRNA and the export of pre-40S subunits from the nucleus, even though it is present in the $90 \mathrm{~S}$ complex. Tsr1 must be added to the pre-40S complex after its release from the $90 \mathrm{~S}$ complex. Many trans-acting factors associated with the pre-40S subunits are released before exiting the nucleus (Jackson et al., 2010; Moy and Silver, 2002; Panse, 2011).

The formation of $60 \mathrm{~S}$ subunits is considerably more complicated than that of the $40 S$ subunit and involves the maturation of three rRNA species, the incorporation of over 30 ribosomal proteins, and requires over 100 trans-acting factors. Maturation of $60 \mathrm{~S}$ subunits begins in the nucleolus but is eventually completed within the cytoplasm. After the pre-40S and pre-60S subunits are formed, the two subunits undergo divergent processing steps on their way to the nuclear pore complex where NESs (nuclear export sequences) present on both pre-subunits are recognized by the export factor Xpo1, also known as Crm1 (Hurt et al., 1999; Moy and Silver, 1999, 2002; Stage-Zimmermann et al., 2000). The molecules of the pre-40S subunit that are recognized by Crm1 include Ltv1, Dim2, and Rio2, the only trans-acting factor with a NES known to be associated 
with pre-60S subunits is Nmd3 (Ribbeck and Gorlich, 2002; Seiser et al., 2006; Zemp et al., 2009).

There are several factors that are not associated with the mature 605 subunit that are part of the late cytoplasmic maturation of pre-60S subunits such as two GTPases: Lsg1/Kre35 and Eif1, two ATPases: Drg1 and Jjj1-Ssa1/Ssa2, a $\mathrm{Zn}^{2+}$ finger protein: Rei1, and a phosphatase: Yvh1 (Panse and Johnson, 2010). Most of these factors prevent the premature joining of $40 S$ and 605 subunits until maturation is completed within the cytoplasm. Of particular interest to this dissertation is the GTPase Efl1. Shwachman-Bodian Diamond syndrome protein, SBDS, along with Efl1 are required for release of the anti-association factor elF6 from pre-60S subunits in the cytoplasm (Menne et al., 2007; Valenzuela et al., 1982; Zemp et al., 2009). The pre-60S subunits that emerge from the nucleus contain the anti-association factor elF6 and the hydrolysis of GTP by Efl1 in association with SBDS is required for elF6 release so $60 \mathrm{~S}$ subunits can participate in translation. This release is also necessary for elF6 to be recycled back to the nucleus to function in transporting more pre-60S subunits to the cytoplasm (Basu et al., 2001; Finch et al., 2011; Gandin et al., 2008; Miluzio et al., 2009; Senger et al., 2001; Wong et al., 2011).

\section{Overview of translation}

Ribosomes and associated factors carry out the process of translation, the means by which nucleotide triplets within a mRNA dictate the synthesis of proteins via the genetic code. Initiation is the process by which appropriate AUG 
codons are identified in mRNAs and $80 \mathrm{~S}$ ribosomes with initiator-tRNA bound to the $\mathrm{P}$ site are poised to begin the process of translational elongation. The most tightly regulated steps of translation are typically found during the initiation phase (Jackson et al., 2010; Sonenberg and Hinnebusch, 2009). There is both a canonical and non-canonical pathway of eukaryotic initiation. The canonical pathway begins with the formation of the ternary complex including elF2, GTP, and an initiator transfer RNA charged with a Met-tRNAMet ${ }_{i}$. This complex joins with a $40 \mathrm{~S}$ subunit already bound to elF1, which together with elF1A, elF3, and elF5 form the $43 S$ pre-initiation complex. The mRNA is recognized through 5'cap structures by the elF4F complex (elF4F bound to elF4E, elF4G, elF4A). This complex recruits the helicase elF4B which can melt RNA secondary structures in an ATP-dependent fashion. The $43 \mathrm{~S}$ pre-initiation complex is then able to bind the mRNA/elF4F complex and begins the process of scanning from the 5' end of the mRNA towards the 3' end until the complex finds a suitable AUG.

When the scanning complex localizes the AUG start codon in what will become the $\mathrm{P}$ (peptidyl) site, elF1 is released, and elF5 facilitates the hydrolysis of elF2-GTP to elF2-GDP. After elF2-GDP is released, the $60 \mathrm{~S}$ subunit is able to join with the 48S complex and elF5B subsequently mediates the release of elF1, elF3, elF4B, elF4F, and elF5. Next, elF5B-GTP is hydrolyzed to elF5B-GDP and elF5B-GDP and elF1A are released. The $80 \mathrm{~S}$ initiation complex is now ready to move to the elongation phase of translation. After translation is complete, 
termination occurs and many of the components of the translational machinery are recycled to participate in translation of other mRNAs (Jackson et al., 2010).

The alternative pathway of initiation involves mRNA elements called internal ribosome entry sites (IRES). Initially identified in viral mRNAs, these structures are able to recruit translational machinery without the normal initiation complex formation. By employing these alternative strategies for translational initiation viruses are able to co-opt a host's translational machinery to their own ends. As is typically the case, it was eventually shown that host cells also employ these alternative strategies for translational initiation, but do so infrequently as forms of regulation at specific points in development, cell cycle progression, or in response to stress (Jackson et al., 2010).

\section{Peptide Bond Formation: Elongation}

Once the $80 \mathrm{~S}$ initiation complex is formed, and the Met- tRNA ${ }^{M_{i}}{ }_{i}$ is in the P site, elongation can commence. The next codon downstream of the initiator AUG is used to specify which aminoacyl-tRNA will productively enter the A (aminoacyl) site of the complex in a ternary complex with EF1A and GTP. Once GTP is hydrolyzed and EF1A is released, a peptide bond is formed between the amino acids, then the ribosome shifts one codon toward the 3' end catalyzed by EF2 and GTP hydrolysis, the A site is open and the newly synthesized peptidyltRNA is translocated to the P site. The deacylated tRNA formerly occupying the P site exits the ribosomal complex through the $E$ (exit) site (Cech, 2000). 
Elongation continues in most cases until the ribosome encounters a stop codon in the A site and termination occurs.

Numerous human diseases have been linked to defects in the production of ribosomes or in the process of translation. The focus of this dissertation will be on diseases linked to defects in ribosome synthesis. The inherited bone marrow failure syndromes as a class appear to be driven to varying extents by defects in the synthesis in ribosomes. Some notable exceptions to this general statement are discussed in the following section.

\section{Bone Marrow Failure syndromes and Other Ribosomopathies}

Inherited Bone Marrow Failure syndromes (IBMFSs) are a heterogeneous array of diseases that have in common a failure to produce one or multiple blood lineages and are often associated with a significant cancer predisposition well above that of the general population. The IBMFS also have certain unique features of presentation and pathophysiology characteristic of their specific disease. The IBMFSs discussed in detail below include Fanconi Anemia (FA), Diamond Blackfan anemia (DBA), Dyskeratosis Congenita (DC), Shwachman Diamond syndrome (SDS), Severe Congenital Neutropenia (SCN), and Pearson Syndrome. Many of these syndromes have molecular defects in ribosome synthesis that contribute to disease pathophysiology and are given the designation of "ribosomopathies." DBA and SDS will be the primary focus of this dissertation. 


\section{Hematopoiesis}

Hematopoiesis refers to the generation of mature blood cells from the long-term hematopoietic stem cell (LT-HSC) progenitor. In adults this takes place in the bone marrow where HSCs divide to form more HSCs, the self-renewing property common to all stem cells, as well as differentiate to form committed progenitors (Metcalf, 2007). Mature cells of the hematopoietic system include lymphocytes, granulocytes, monocytes, megakaryocytes, and erythrocytes. Lymphocytes include both $T$ and $B$ cells central to immune system function. $T$ cells are antigen recognition and antigen presenting cells whose final maturation takes place in the thymus, while B cells fully differentiate in the bone marrow and produce antibodies important in phagocytosis and the complement system of immunity. Granulocytes, also called polymorphonuclear cells (PMN), include eosinophils released to sites of allergy or parasitic infection. Neutrophils are phagocytic and engulf pathogens such as opsonized bacteria and use their specialized set of enzymes such as NADPH oxidases and myeloperoxidases (MPO) to generate ROS in phagolysosomes and destroy the bacteria. Basophils respond to inflammation and release heparin, an anticoagulant. Monocytes differentiate into macrophages and dendritic cells in the spleen and function by phagocytosing pathogens. The most common while blood cells in circulation are neutrophils. Megakaryocytes release platelets important in forming blot clots. Erythrocytes are the transporters of hemoglobin, the primary oxygen carrier in the body (Metcalf, 2007). 
The first maturation step from the hematopoietic stem cell splits the lineages for lymphocytes (common lymphoid progenitor, or CLP) and all other blood cell types (common myeloid progenitor, or CMP). The CLP will then differentiate into a lineage specific pre-B cell or pre-T cell. The differentiation of the myeloid lineage is much more complex. The CMP first becomes either a MEP (megakaryocyte erythroid progenitor) or a GMP (granulocyte macrophage progenitor). The MEP further differentiates into Meg-CFC and then megakaryocytes, which generate platelets, or BFU-E (burst forming units erythroid). The BFU-E give rise to CFU-E (colony forming units erythroid) and eventually mature erythrocytes. The GMP further differentiates into either the Mast-CFC that will become a basophil, the eosinophil CFC (Eo-CFC), or the granulocyte macrophage CFC (GM-CFC). The GM-CFC differentiates into two lineages, the granulocyte CFC (G-CFC) that will become neutrophils or the macrophage CFC (M-CFC) that will become monocytes (Metcalf, 2007). The process of differentiation is dependent not only on cytokines or growth factors that drive the formation of the various blood lineages, but also the complex stromal environment of the bone marrow niches through an interplay of multiple types of regulation of numerous cell types in the marrow (Bianco, 2011). Recent evidence obtained from mouse studies indicated that there are several classes of LT-HSCs with predispositions for differentiation toward specific blood lineages, adding additional levels of complexity (Cavazzana-Calvo et al., 2011). Inherited bone marrow failure syndromes all have a pathological defect in one or multiple steps during the maturation process outlined above. 


\section{Diamond Blackfan Anemia: Pathophysiology}

Diamond Blackfan anemia (DBA) is a macrocytic normochromic anemia that most commonly manifests in infancy. In addition to red blood cell hypoplasia, up to $40 \%$ of patients also have a congenital defect, with $25 \%$ having multiple anomalies. The most common anomalies are short stature, craniofacial defects, triphalangeal or bifid thumbs, and deformities of the urogenital and cardiovascular systems (Vlachos et al., 2008). The first link between DBA and the ribosome came in 1999 when a chromosomal translocation in a DBA patient was shown to inactivate RPS19, (Ribosomal Protein of the Small subunit number 19) a gene encoding a structural component of the $40 \mathrm{~S}$ ribosomal subunit (Draptchinskaia et al., 1999). RPS19 remains the most common target of inactivating mutations in DBA, compromising approximately $25 \%$ of the patient population. To date, pathogenic mutations have been identified in nine additional genes encoding ribosomal proteins of both the large (60S: RPL5, RPL11, RPL35a, RPL26) and small (40S: RPS7, RPS10, RPS17, RPS24, RPS26,) subunit (Farrar et al., 2011; Gazda et al., 2012; Vlachos et al., 2008). In each case, mutations identified are found in the heterozygous state indicating that DBA is the result of haploinsufficiency for the affected ribosomal protein. The view of DBA as a ribosomopathy became inexorable when the gene responsible for the refractory anemia in patients with the myelodysplastic $5 q^{-}$syndrome was shown to encode ribosomal protein S14 (RPS14) (Ebert et al., 2008). Only limited genotype/phenotype relationships have been identified so far in DBA patients. The most notable is that patients with RPL5 and RPL11 mutations in 
general display a much more severe phenotype including a high prevalence of cleft lip and cleft palate.

Though it has been over a decade since DBA pathogenesis was originally linked to deficiencies of ribosomal proteins, the mechanisms underlying disease pathophysiology, in particular the tissue specificity of disease presentation remain unclear. It has been well established that reduced quantities of a ribosomal protein can interfere with the assembly of the subunit to which it is a part. For example, cell culture models have shown that reducing the level of expression of RPS19 interferes with the biogenesis of $40 S$ subunits and gives a well-defined signature of RPS19 dysfunction in pre-rRNA processing. This prerRNA processing signature associated with RPS19 deficiency is also evident in cells from the bone marrow of DBA patients (Flygare et al., 2007). Pre-rRNA processing signatures have been associated with other ribosomal proteins affected in DBA. The specific signature for a given ribosomal protein will depend on which rRNA (or pre-rRNA) it becomes initially associated with, and also, in the case of the larger rRNAs, which sub-assembly domain of the rRNA contains the protein. Together, these data indicate that ribosome assembly is defective in individuals haploinsufficient for ribosomal proteins, and defective ribosome assembly lies at the heart of DBA pathophysiology.

\section{Molecular Mediators of DBA}

During normal ribosome biogenesis, ribosomal proteins and rRNA are joined in a specific temporal and spatial sequence to form mature ribosomal 
subunits (Warner and McIntosh, 2009). In order to coordinate the generation of ribosomes with the needs of the cell, there are multiple connections between ribosome synthesis and cell division, nutrient availability, and apoptosis (Dai and Lu, 2008; Dai et al., 2004; Fernandez et al., 2002; Lindstrom et al., 2007; Sun et al., 2007). Ribosomal proteins that are synthesized in excess of that needed for subunit assembly are frequently rapidly degraded (Lam et al., 2007). It has now become clear that certain ribosomal proteins have novel, extra-ribosomal, functions when they are not incorporated into ribosomal subunits (Warner and Mclntosh, 2009). Several of these ribosomal proteins influence the delicate balance between cell survival and apoptosis through their interactions with the oncogene MDM2 (murine double minute two oncogene, whose human ortholog is HDM2). MDM2 is an E3 ubiquitin ligase that can target p53 for proteosomal degradation and is one of the exquisitely sensitive primary regulators of p53 stability (Clegg et al., 2008). RPL11, RPL5, RPL23, RPL26, RPS3, and RPS7 are all capable of binding MDM2 during "ribosomal stress." Ribosome stress can be induced by a variety of effectors including treatement with actinomycin $D$, an RNA polymerase I inhibitor. Under these conditions ribosomal proteins are free to interact with and inhibit MDM2 resulting in p53 stabilization and activation (Chen et al., 2007; Dai and Lu, 2004; Dai et al.; Dai et al., 2004; Fumagalli et al., 2009; Horn and Vousden, 2008; Ofir-Rosenfeld et al., 2008; Sun et al., 2007; Yadavilli et al., 2009; Zhang et al., 2003; Zhu et al., 2009).

In addition to ribosomal proteins, there is growing evidence to suggest that $5 \mathrm{~S}$ rRNA plays a role in ribosome stress signaling. The $5 \mathrm{~S}$ rRNA is distinct in that 
it is transcribed by RNA polymerase III independent of the rDNA repeat as transcribed by the polycistronic RNA polymerase I transcript containing the other three rRNAs. 5S rRNA forms a complex with RPL5, and possibly RPL11, prior to the incorporation of this subcomplex into the assembling 60S subunit (Zhang et al., 2007). Intriguingly, the subcomplex of RPL5, RPL11, and 5 S rRNA binds more effectively to MDM2 than each of the individual components alone. The synergistic interaction of this subcomplex with MDM2 could potentially play a major role in signaling abortive assembly of the $60 \mathrm{~S}$ ribosomal subunit leading to p53 stabilization and activation as shown in Fig 2. This observation is potentially of fundamental importance to mechanisms underlying DBA pathophysiology, because erythrocyte precursors in the bone marrow of patients show a proapoptotic phenotype which appears linked to p53 activation (Lipton et al., 1986; Nathan et al., 1978; Ohene-Abuakwa et al., 2005; Perdahl et al., 1994). Thus, this pathway is hypothesized to be the cornerstone linking defective ribosome assembly to the pro-apoptotic phenotype of erythroid progenitors in the marrow of patients with DBA. 


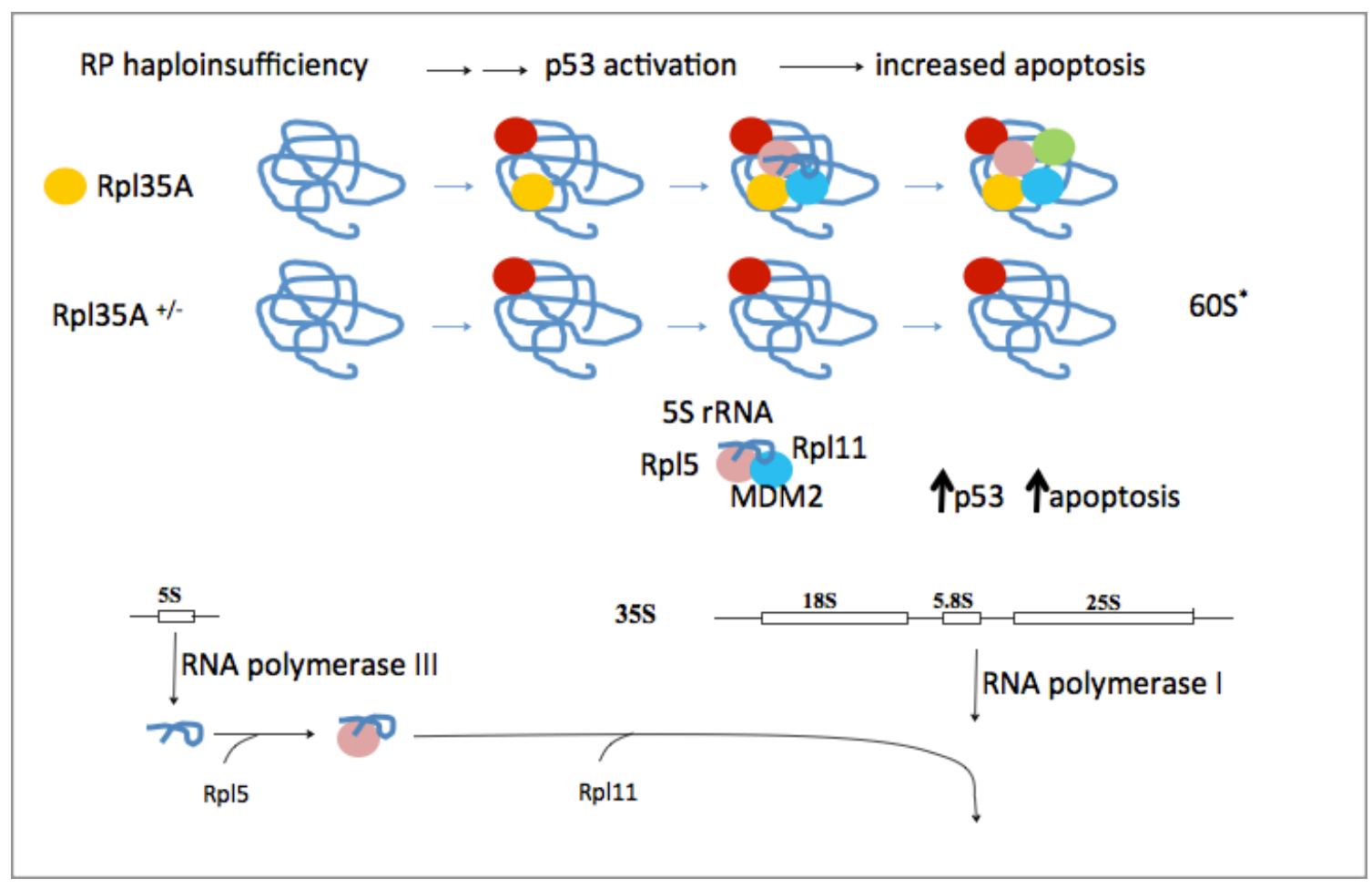

Fig. 2: The 5S rRNA subcomplex as a critical mediator of ribosome stress signaling to p53 activation.

When ribosomal proteins become limiting, such as in the setting of ribosomal haploinsufficiency in DBA pathogenesis, ribosomal proteins are free and are capable of interacting with MDM2 leading to p53 activation. In the setting of haploinsufficiency for a large subunit ribosomal protein the 5S rRNA subcomplex may play a critical role in ribosomal stress signaling. It is less clear which factors play vital roles in ribosomal stress signaling when small subunit ribosomal protein genes are affected in DBA or when mutations are found in either the RPL5 or RPL11 genes (Lipton and Ellis, 2009). 


\section{Previous Animal Models of DBA}

Generation of an animal model that recapitulates both the hematological and ribosomal defects associated with DBA patients has proven challenging. A knockout mouse would seem the obvious choice in a disease marked by haploinsufficiency for ribosomal protein genes. The heterozygous Rps19 mouse model, showed no detectable phenotypes, and the homozygous mutant led to early embryonic lethality (Matsson et al., 2004). The first animal models of DBA that showed a blood lineage defect were created in zebrafish (Danilova et al., 2008; Uechi et al., 2008). Zebrafish have several advantages as an animal model for hematology. The transparent embryos make iron-containing substances, such as the heme in the hemoglobin of mature red blood cells, easily visualized. The externally fertilized embryos provide an easy conduit for over or under expressing a gene of interest. Zebrafish models have allowed for high throughput drug screening and localization of genetic modifiers. Though many advances have been made possible through study of zebrafish models, there are also several important differences in zebrafish blood development when compared to human processes. The sites of blood lineage development in zebrafish are far removed from their localization in humans. Hematopoietic stem cells (HSCs) in zebrafish are found in kidney marrow rather than the bone marrow as in mammals (Carradice and Lieschke, 2008; Jing and Zon, 2011).

The first mammalian model of DBA with an erythroid defect was serendipitously discovered during a screen for mutations that cause dark-skinned footpads in mice (McGowan et al., 2008). Two causative mutations in the screen 
were identified as ribosomal protein genes, Dsk3 as Rps19, and Dsk4 as Rps20. Only Rps19 is a known DBA gene. After this discovery, the authors identified a very mild anemia in the affected mice. Subsequently, they reversed pathological findings in an Rps6 heterozygous knockout mouse by inhibition of p53 function, a common theme recognized among putative animal models of DBA (Ball, 2011; Boultwood et al., 2011; Danilova et al., 2008; Jaako et al., 2011; McGowan et al., 2011). The validity of an Rps6 heterozygous mouse to study DBA is diluted by the lack of an identified mutation in RPS6 in a DBA patient, as well as the specialized function of Rps6 as a downstream target fr phosphorylation by the mTOR pathway (Magnuson et al., 2012).

A more recent mouse model expressed a Rps19R62W point mutation found in a DBA family pedigree. It has been hypothesized that this mutation may function in a dominant negative manner (Devlin et al.). This particular model linked inducible expression of this modified allele in a normal mouse genetic background to some of the blood lineage defects seen in DBA patients but also differed in certain important respects. These mice lack some of the key prerRNA processing defects seen in DBA patients with known RPS19 mutations, as well as a block in blood progenitor maturation at a different stage of development than those seen in bone marrow specimens from DBA patients (Flygare et al., 2007). Thus, none of the currently available animal models of DBA are entirely adequate and improvements are still needed. Our contribution to this gap are represented in chapter two of the appendix, which details our role in the 
validation of ribosome defects in a novel mouse model of DBA (Jaako et al., 2011).

\section{Shwachan Diamond syndrome (SDS)}

Shwachman Diamond syndrome typically presents in early infancy, but has also been diagnosed in adolescents. The most common presenting symptom is malabsorption of fat from the diet due to pancreatic insufficiency and the associated decrease in amylase, trypsinogen, and lipase production. The first case of SDS was described as pancreatic insufficiency with bone marrow dysfunction and the two symptoms remain the diagnostic criteria in the present day (Rothbaum et al., 2002; Shimamura and Alter, 2010; Shwachman et al., 1964). Most patients have a neutropenia that upon analysis of bone marrow samples reveals a hypocellular bone marrow with decreased myeloid progenitors, but the initial neutropenia often presents later than the symptoms of malabsorption. The neutropenia severity shows a wide range of variation among patients (Shimamura and Alter, 2010). In addition to neutropenia, multiple other blood lineages can be affected in SDS, with more severe disease courses leading to aplastic anemia, eventual myelodisplastic syndrome (MDS), or acute myelogenous leukemia (AML) (Berthou et al., 1991; Shimamura and Alter, 2010). The most frequent causes of death in SDS patients are sepsis, due to the neutropenia, or AML. Unfortunately the median age of survival is currently 36 years old (Shimamura and Alter, 2010). 


\section{SDS Genetics}

Biallelic mutations in SBDS (Shwachman Bodian Diamond Syndrome) have been identified in over $90 \%$ of SDS patients (Boocock et al., 2003). The disease is inherited in an autosomal recessive pattern. There are no cases associated with homozygous null mutations and homozygous loss of Sbds in mouse models is embryonically lethal (Zhang et al., 2006). Most mutations are thought to be hypomorphic alleles that arose from conversion with an adjacent pseudogene SBDSP (Shammas et al., 2005). The most common mutations are in exon 2, and include c.183_184insCT or c. $258+2 \mathrm{~T}>\mathrm{C}$ on separate genes, or the combination of the two mutations in the same gene. Seventy-six percent of patients have at least one of these three mutations and $62 \%$ have both (Boocock et al., 2003).

\section{$\underline{\text { SBDS Protein Functions }}$}

The SBDS protein is evolutionarily conserved across eukaryotes and many archaebacteria (Boocock et al., 2003; Menne et al., 2007; Ng et al., 2009; Shammas et al., 2005). First clues as to the function of SBDS came from the finding that certain archaebacterial orthologs were found in operons containing genes involved in RNA metabolism. Included among these genes were ones encoding proteins involved in pre-rRNA processing. Studies on the yeast ortholog of SBDS, Sdo1, revealed a role for Sdo1 in the maturation of 605 ribosomal subunits (Menne et al., 2007). Specifically, Sdo1 plays a role in releasing the anti-association factor Tif6 from nascent pre-60S subunits entering 
the cytoplasm. The failure to release Tif6, also limits its recycling back to the nucleus where it is involved in the nucleocytoplasmic transport of pre-60S subunits. These combined effects severely limit the production of functional $60 \mathrm{~S}$ subunits in cells depleted of Sdo1. Recent studies in mammalian cells have shown related functions for SBDS in the maturation of 605 ribosomal subunits (Wong et al., 2011).

In addition to its role in the maturation of 60S ribosomal subunits, SBDS has been implicated in a number of additional cellular processes The SBDS protein has been shown to colocalize with the mitotic spindle, and cell models expressing mutant versions of the protein analogous to SDS patient mutations have been shown to have altered SBDS cellular localization. This is proposed as an instigator of genomic instability in SDS, a putative pathway leading to AML (Austin et al., 2008; Maserati et al., 2009; Orelio et al., 2009). Changes in cellular localization are hypothesized to occur via modifications in the SUMOlyation domain at the C-terminus of the protein (Orelio et al., 2011). Bone marrow progenitor cells show an increased propensity for apoptosis, and cellular models of SDS have shown an increased sensitivity to Fas (a ligand for death domain receptors that activate downstream caspase cleavage and result in apoptosis) and changes in cellular localization of Fas when stimulated (Rujkijyanont et al., 2009; Rujkijyanont et al., 2008; Watanabe et al., 2009).

Other changes in cell function with mutations in SBDS include the decreased ability of neutrophils to polymerize F-actin in response to appropriate cellular stimuli (Orelio and Kuijpers, 2009), increased reactive oxygen species 
(ROS) production (Ambekar et al., 2010), and changes in the bone marrow stromal environment (Raaijmakers et al., 2010). Bone marrow from SDS patients show an intrinsic defect in the maturation of blood progenitors (Dror and Freedman, 1999). The dyscrasias found in patient bone marrow samples are due to defects not only in the hematopoietic cells, but also in the stromal cells that support the differentiation of stem cells as evidenced by bone marrow transplant studies in animal models (Rawls et al., 2007). Clearly, any of these multiple functions of the SBDS protein could contribute to the clinical features of SDS.

\section{Dyskeratosis Congenita (DKC)}

Dyskeratosis Congenita occurs in X-linked as well as autosomal dominant and autosomal recessive inheritance patterns. Clinical findings include dystrophic nails, oral leukoplakia, lacy reticular pigmentation, lacrimal duct stenosis, sparse and/or gray hair, poor dentition, development delay, esophageal stenosis, and avascular necrosis of the hip (Alter, 2007; Berthou et al., 1991; Liu and Ellis, 2006; Narla and Ebert, 2010; Savage and Alter, 2009; Savage et al., 2009; Tamary et al., 2010). Criteria for diagnosis include measurement of telomere length, as DKC patients usually have telomeres that fall within the very lowest percentile of telomere length for age matched controls. With age their risk for aplastic anemia and MDS increases much more dramatically than the rest of the population (Alter et al., 2010; Alter et al., 2009; Du et al., 2009; Gadalla et al., 2010; Heiss et al., 1998; Savage and Alter, 2008, 2009; Savage et al., 2009; 
Tamary and Alter, 2007; Tamary et al., 2010). Bone marrow inspection usually reveals hypocellular bone marrow with decreased megakaryocytes. Bone marrow failure is the leading cause of death in DKC (Narla and Ebert, 2010). Pathogenic mutations in DKC patients have been identified in six genes that all function in the maintenance of telomeres. Many of the genes mutated in DKC produce products that perform multiple functions in the cell. Mutations in DKC1 have an X-linked recessive inheritance pattern, and this particular mutation leads to defects in ribosome biogenesis due to the functions of dyskerin as a member of the snoRNP complex that is required for psuedouridylation of pre-rRNA. In addition to its function in maturation of rRNA, dyskerin is also associated with other proteins, such as TERT, and RNAs, such as TERC, to form the telomerase complex that protect the telomeres found at the end of chromosomes (Heiss et al., 1998; Knight et al., 1998). Mutations in TERT can be inherited in autosomal dominant and autosomal recessive inheritance patterns, while TINF2 and TERC have only been shown in autosomal dominant patterns, and NOP10/NOLA3 and NHP2/NOLA2 are autosomal recessive. Except for the mutations in dyskerin none of the other mutations in DKC genes are thought to affect ribosome function. Interestingly, the X-linked form of the disease is typically more severe than the other forms indicating that effects on ribosome synthesis can modify the clinical phenotype in this, and perhaps other disorders. 


\section{Cartilage Hair Hypoplasia $(\mathrm{CHH})$}

Cartilage Hair Hypoplasia $(\mathrm{CHH})$ was first described as a dwarfism in an isolated Amish population (McKusick et al., 1965). In such isolated Amish populations and certain Finnish populations there is an increased incidence of $\mathrm{CHH}$, but it is very rare in the general population (Makitie, 1992; Ridanpaa et al., 2002). It most commonly presents with skeletal dysplasias, immune dysfunction, hypoplastic hair, and increased cancer susceptibility. One or multiple blood lineages can be affected, with cells of the myeloid lineage being most commonly affected (Ganapathi and Shimamura, 2008). Autosomal recessive inheritance patterns are seen with mutations in RMRP encoding the RNA component of RNAse MRP (mitochondrial RNA processing complex). This RNase has a number of targets including the cyclin B2 mRNA, mitochondrial RNA, and a cleavage within ITS1 of $45 S$ pre-rRNA giving rise to the mature $5^{\prime}$ end of $5.8 S$ rRNA. Mutations in RMRP that predominantly affect pre-rRNA processing most commonly cosegregate with skeletal dysplasias, whereas mutations that selectively affect cyclin B2 mRNA cleavage give rise to the immune deficiencies (Theil et al., 2011). Management is primarily supportive care, but transplant may be indicated in very severe cases (Narla and Ebert, 2010).

\section{Treacher Collins syndrome (TCS)}

Treacher Collins syndrome (TCS) is usually recognized shortly after birth due to its characteristic set of facial features and was initially described by an ophthalmologist over a century ago (Collins, 1900). Deformations are caused by 
inadequate migration of neural crest cells during fetal development (Dixon et al., 2006) leading to downward lateral slanting palpebral fissures (outer corners of the eyelid), abnormal ear development with hearing loss as a common side effect, hypoplasia of the maxillary and malar portions of the skull leading to a flattening of the mid-face and micrognathia (abnormally small jaw) and cleft palate (Narla and Ebert, 2010). The management of these patients requires the attentive care of numerous types of physicians due to the complications that are associated with such physical anomalies (Posnick and Ruiz, 2000). Many of the craniofacial defects seen in TCS are reminiscent of those seen with RPL5 and RPL11 mutations in DBA (Narla and Ebert, 2010).

Mutations in the gene TCOF1 that encodes the protein treacle were linked to this autosomal dominantly inherited condition (Jill Dixon, 1996). Treacle binds to rDNA and upstream binding factors required for the transcription of the $45 \mathrm{~S}$ polycistronic pre-rRNA sequence (Valdez et al., 2004). It also appears necessary for methylation of the $45 S$ pre-rRNA. More recently, mutations in genes encoding critical shared polypeptides between RNA polymerase I and III have been identified in TCS patients (Valdez et al., 2004). This observation indicates that defects in RNA polymerase I transcription likely underly disease pathophysiology. A mouse model haploinsufficient for Tcof1 demonstrated that the defects in neural crest cell migration and neural epithelial apoptosis are instrumental in creating the physical findings pathognomonic of TCS (Dixon et al., 2006). The craniofacial defects can be rescued by inhibiting p53 function during fetal development, even if the ribosomal defects are not corrected (Jones et al., 2008). 
One of the more surprising aspects of the relationship between TCS and DBA is the fact that TCS patients do not exhibit bone marrow failure. The pathophysiologic mechanism underlying current views of DBA involve free ribosomal proteins interacting with MDM2 to activate p53. Much of the experimental support for this mechanism comes from in vitro cell culture work using Actinomycin D, an inhibitor of RNA polymerase I (Aktipis and Panayotatos, 1981; Casse et al., 1999). Nevertheless, these studies seem more relevant to the pathophysiologic mechanisms underlying TCS. How then can we reconcile the fact that investigators in both fields appear to be employing the same pathophysiologic mechanisms for distinct diseases? While there is some overlap in clinical presentation between the two disorders, specific craniofacial anomalies observed in DBA are restricted to specific ribosomal protein genotypes, whereas, potentially even more critically TCS lacks an associated bone marrow failure (Shimamura and Alter, 2010). Clearly, investigators are missing something when ribosomal protein defects give rise to one disease and ribosomal RNA defects give rise to another and both disease phenotypes can be rescued by inactivating p53.

\section{Fanconi Anemia (FA)}

Fanconi Anemia, FA, is the most commonly diagnosed IBMFS (Shimamura and Alter, 2010). It presents most commonly as pancytopenia with a hypocellular bone marrow. Other diagnostic features include an increased MCV (mean corpuscular volume) and increased $\mathrm{HbF}$ (fetal hemoglobin) (Ameziane et 
al., 2008; Soulier, 2011). More than $60 \%$ of patients have at least one congenital anomaly; the most common of which is short stature followed by café au lait spots, and hypo or hyperpigmentation. Approximately one third of patients have radial defects involving the thumbs (Shimamura and Alter, 2010; Soulier, 2011). The cancer risk for Fanconi Anemia patients is 50 times higher than the general population, and the risk for MDS is increased 5,000 times. The most common malignancies in order of incidence are AML, head and neck squamous cell carcinoma, liver cancers, and vaginal squamous cell carcinoma (Rosenberg et al., 2004; Rosenberg et al., 2005).

Thirteen genes have been identified as FA genes. With the exception of FANCB, which is $\mathrm{X}$-linked, the remainder are inherited in an autosomal recessive fashion (FANCA, FANCC, FANCD1/BRCA2, FANCD2, FANCE, FANCF, FANCG/ XRCC9, FANCJ, FANCJ/BACH1/BRIP1, FANCL, FANCM, FANCN/PALB2). All of the FA linked genes are known to function in the repair of interstrand crosslinks in DNA (Alter et al., 2007; Andreassen et al., 2004; Bakker et al., 2009; de Winter and Joenje, 2009; Fagerlie et al., 2001; Meetei et al., 2003; Moldovan and D'Andrea, 2009; Nakanishi et al., 2002; Pichierri et al., 2004; Singh et al., 2009; Taniguchi et al., 2002; Wang, 2007; Wang et al., 2007). None of the Fanconi genes are known to function in ribosome synthesis. Diagnostic criteria for FA include testing of patient derived cells for extreme sensitivity to DNA crosslinking agents, followed by sequencing of the known FA genes. Hematopoietic stem cell transplant is the only curative therapy for this disease, though it only corrects the anemia. 


\section{Severe Congenital Neutropenia (SCN)}

Severe congenital neutropenia patients are usually diagnosed due to an infection, abscess, or pneumonia. The only defect associated with this illness identified presently is a defect in the promyelocyte/myelocyte stage of differentiation of neutrophil precursors. Multiple genes have been linked to SCN but the two most commonly affected are ELA2 or ELANE genes. ELANE and ELA2 encode neutrophil elastase enzymes which are the major secretory proteins in neutrophils (Dale et al., 2006; Dale et al., 2000; Grenda et al., 2007). The heterozygous mutations found associated with $\mathrm{SCN}$ are typically missense mutations that interfere with protein folding in the endoplasmic reticulum. Because the overall secretory load is so great in neutrophils and these proteins represent such a high fraction of the total secretory pool, their misfolding causes ER stress of such severity that maturation arrest occurs and cells undergo apoptosis (Germeshausen et al., 2009a; Germeshausen et al., 2009b; Parikh and Bessler, 2012; Skokowa et al., 2009; Tamary et al., 2010; Welte and Zeidler, 2009; Xia and Link, 2008; Zeidler et al., 2009). Treatment with G-CSF (granulocyte-colony stimulating factor) or stem cell transplantation for patients that don't respond are the current standards of care for this disorder (Shimamura and Alter, 2010).

\section{Other Less Common IBMFS}

Pearson syndrome (PS) is another IBMFS that is characterized by anemia and exocrine pancreatic insufficiency, originally described in 1979 (Pearson et al., 
1979). It is often fatal in infancy. Patients that survive past early neonatal life develop symptoms similar to Kearns-Sayre Syndrome, which is a mitochondrial myoencephalopathy (a disorder of muscle and brain tissues) (McShane et al., 1991; Rotig et al., 1990; van den Ouweland et al., 2000). Patient samples were found to have large deletions and/or rearrangements in mitochondrial DNA which disrupt energy metabolism. This preferentially affects tissues requiring a high threshold of mitochondrial function like the central nervous system and skeletal muscle (Baerlocher et al., 1992; Blaw and Mize, 1990; Cormier et al., 1990; Kleinle et al., 1997; Lee et al., 2007; Niaudet et al., 1994; Santorelli et al., 1996; van den Ouweland et al., 2000).

The bone marrow failure and pancreatic insufficiency in Pearson syndrome is reminiscent of SDS, but there are significant differences. Pearson syndrome patients have a sideroblastic anemia. Ringed sideroblasts are nucleated red cells that did not mature before leaving the bone marrow. They produce a granular staining with dyes to visualize iron, because iron granules form deposits in the mitochondria around the nucleus in these cells. This perinuclear arrangement is what forms the ring of the ringed sideroblast. Sideroblasts are also evident in X-linked sideroblastic anemia caused by defects in the gene encoding $\delta$-amino levulinic acid synthase. This enzyme is mitochondrial and performs the first step in the synthesis of heme. The iron deposits formed in this disease result from the lack of protoporphyrin IX production. In the absence of protoporphyrin IX, iron that would normally be used for heme synthesis deposits within the organelle giving rise to the ringed 
sideroblasts (Cotter et al., 1992; Cotter et al., 1994; Ferreira, 1993; Mantzourani et al., 1995; Pagon and Bird, 1993; Pearson et al., 1979; Raskind et al., 1991; Sutherland et al., 1988). Mitochondrial dysfunction in bone marrow progenitors in Pearson's syndrome presumably disrupts protoporphyrin IX synthesis, explaining the sideroblasts seen in this disease. Also, SDS patients have a hypocellular bone marrow and fatty infiltration of the pancreas, while Pearson Syndrome patients usually have normocellular bone marrow with fatty vacuolization (Blatt et al., 1994) and fibrotic changes in the pancreas (Shimamura and Alter, 2010). In contrast, there are no reports of sideroblasts associated with anemia in Shwachman Diamond syndrome patients. While these observation emphasize the differences between Pearson Syndrome and SDS, we feel that the work described in this dissertation indicates it may be worthwhile to look more carefully at the relationship between these two disorders. Amegakaryocytic thrombocytopenia (CAMT) is a deficiency of megakaryocytes whose normal function is to produce platelets. Children usually present with symptoms of a clotting disorder such as infants with excessive bruising. It Is not uncommon for patients to be diagnosed as adolescents or adults after development of a secondary condition such as aplastic anemia or MDS without prior recognition of the initial deficit in megakaryocytes (Shimamura and Alter, 2010). Biallelic mutations in the gene encoding the thrombopoietin receptor, MPL, have been found in the majority of patients (Ihara et al., 1999). In a knockout mouse model deficient in $\mathrm{mpl}$ it was discovered that the receptor, in addition to its function in the production of megakaryocytes (Bartley et al., 1994; 
de Sauvage et al., 1994; Gurney et al., 1994; Kaushansky et al., 1994), is also central to the maintenance of pluripotent bone marrow progenitors (Alexander et al., 1996a; Alexander et al., 1996b).

Thrombocytopenia absent radii is another thrombocytopenia that differs from CAMT because of the associated congenital defect in its title. These patients lack radii bilaterally but still develop thumbs, though they are nonfunctional (Thompson et al., 2001). This is an important distinguishing characteristic from FA in which patients without radii always have associated thumb defects (Shimamura and Alter, 2010).

\section{Dissertation Overview}

As a group, IBMFS show multifactorial pathophysiology with frequent links to ribosome biology. Significant overlap in the clinical features of these disorders tends to complicate diagnoses, making it difficult to differentiate one from another based only on clinical parameters. Considerable insight into molecular underpinnings of these diseases has been gained in recent years but many questions remain unanswered. Further, this understanding at the molecular level has not translated into dramatic improvements in diagnostic modalities, improved treatments, or substantial improvements in various prognostic parameters. This dissertation will focus on two specific IBFMS both of which arise to varying extents from defects in ribosome synthesis. The first portion of this dissertation will discuss the identification of a new ribosomal protein gene involved the pathogenesis of DBA and provide a facile assay to screen patients for mutations 
in genes encoding large subunit ribosomal proteins. These studies also investigate the putative molecular mediators responsible for the increased p53 activation shown in numerous DBA models.

The second portion of the dissertation will focus on SDS. It will include descriptions of generation of a mammalian model for SDS in an erythroleukemic human cell line and the functional consequences of depleting cells of SBDS. The concepts envisioned in this chapter were the direct result of work performed in a yeast model of SDS developed by Dr. Joseph Moore IV and Dr. Steve Ellis that first identified the changes in respiration associated with loss of SDO1, the yeast ortholog of SBDS. This part of the dissertation investigates a potential link between changes in cellular metabolism and mitochondrial dysfunction in SDS that has not been previously examined.

Given the heterogeneous nature of IBMFS several methods of investigation were initially pursued before the particular methods used in the body of the dissertation were chosen. Those portions of my laboratory work not contained within the body of the text are located in appendix one. This includes previously published work on an inducible shRNA mouse model that led to decreased RPS19 protein expression and is arguably the most phenotypically accurate animal model for DBA developed to date (Jaako et al., 2011) as well as additional work in the mES cell model introduced in chapter three that did not easily fit within the description of the mechanisms described in that chapter. 


\section{CHAPTER II}

\section{MATERIALS AND METHODS}

\section{Human Sample Preparation}

All human samples were collected using protocols necessary for the protection of human subjects and in compliance with institutional guidelines. Whole blood samples (ranging in volume from 1.5-8 ml) were collected by personnel at the patient's representative institution in vacutainer tubes treated with sodium heparin to prevent clotting. The samples were then catalogued and shipped to the University of Louisville devoid of identifying patient characteristics. Initial experiments were performed on samples from the proband, her unaffected parents, and a healthy volunteer all of which were collected at Loma Linda University. Subsequent samples including patients with characterized RPL5 and RPL35A mutations were obtained from Dr. Adrianna Vlachos at the Feinstein Institute for Medical Research in New York and Dr. Jason Farrar at Johns Hopkins University, respectively.

\section{Buffy Coat Preparation of Mononuclear Cells and Stimulation with ConA}

All samples involving human blood were treated as potentially hazardous materials and necessary precautions were taken. All centrifugation steps were 
carried out at room temperature. Whole blood was removed from vacutainer tubes and placed in sterile $50 \mathrm{ml}$ conical tubes. The blood was then diluted with an equal volume of sterile PBS (Mediatech, VWR). Three $\mathrm{ml}$ of Ficoll reagent (GE Healthcare) was added to the bottom of $15 \mathrm{ml}$ conical tubes and $4 \mathrm{ml}$ of diluted blood carefully layered onto the Ficoll at an angle to prepare an appropriate interface and maximize cell separation. Aliquots of each sample were centrifuged at $400 \times g$ for 40 minutes with the acceleration set to 1 and the brake set to 0 . All but $1 \mathrm{ml}$ of the serum layer on the top of the gradient was aspirated with a $5 \mathrm{ml}$ pipette and then the buffy coat (white fluffy phase) containing mononuclear cells was collected very carefully and slowly to avoid contamination of the buffy coat with other cellular layers. The resulting buffy coat was placed in a fresh $15 \mathrm{ml}$ sterile conical tube and resuspended in a total volume of $14 \mathrm{ml}$ with sterile PBS. An aliquot was taken from this solution for counting in a 1:1 solution with trypan blue (MP Biomedicals) in a hemocytometer (Hausser Scientific) to determine cell numbers in each sample.

The cells were harvested by centrifugation at $1500 \mathrm{rpm}$ for 10 minutes and then suspended in RPMI-C. RPMI-C was generated from RPMI 1640 (Mediatech, VWR) with the addition of 10\% FBS (Mediatech-VWR, heat inactivated), $50 \mathrm{U} / \mathrm{ml}$ penicillin (Invitrogen), $50 \mu \mathrm{g} / \mathrm{ml}$ streptomycin (Invitrogen), 0.01M HEPES pH 7.4 (Invitrogen), and 2 mM L-glutamine (GlutamaxInvitrogen). Immediately before use, concanavalin A (ConA), was added to a final concentration of $5 \mu \mathrm{g} / \mathrm{ml}$ (Sigma Aldrich, prepared as a stock in RPMl at 5 $\mathrm{mg} / \mathrm{ml}$, stored at $-20^{\circ} \mathrm{C}$, and diluted $1: 1000$ for use). Cells were diluted to a 
concentration of $2 \times 10^{6}$ cells $/ \mathrm{ml}$. Cells were stimulated with ConA for 96 hours at $37^{\circ} \mathrm{C}$ with $5 \% \mathrm{CO}_{2}$ in air in a humidified atmosphere. After 96 hours cells were harvested using QIAshredder columns to lyse cells and remove cell debris. An AllPrep kit from QIAgen was used for the extraction of RNA, DNA, and protein from the samples. The manufacturer's instructions were followed with the following modifications: fresh $\beta$-mercaptoethanol was added with each cell preparation rather than adding to the stock solution, RNA was eluted in a final volume of $30 \mu \mathrm{l}$ and DNA was eluted in a final volume of $100 \mu \mathrm{l}$.

\section{Northern Blotting}

RNA was quantified using the Take3 Synergy plate-reader system (Applied Biosystems) and equal amounts of RNA were fractionated by electrophoresis on $1.5 \%$ formaldehyde-agarose gels as previously described (Flygare et al., 2007). Typically, $5-10 \mu \mathrm{g}$ of total RNA in a final volume of $9 \mu \mathrm{l}$ of diethylpyrocarbonatetreated (1\%) water, was mixed with $33 \mu \mathrm{l}$ of RNA sample buffer: $1.2 \mathrm{X}$ MOPS (10X MOPS; 0.4M 3-(N-morpholino) propane-sulfonic acid, $100 \mathrm{mM}$ sodium acetate, and $10 \mathrm{mM}$ EDTA adjusted to $\mathrm{pH} 7$ with sodium hydroxide), $7.9 \%$ formaldehyde, $60 \%$ formamide, and $0.06 \mathrm{mg} / \mathrm{ml}$ ethidium bromide. After mixing the samples were heated to $65^{\circ}$ for 15 minutes and $4 \mu \mathrm{RNA}$ dye ( $50 \%$ glycerol, $1 \mathrm{mM}$ EDTA, $0.4 \%$ bromphenol blue, $0.4 \%$ xylene cyanol) was added. RNA fractionated in this manner stains with ethidium bromide in the sample buffer so there is no need for subsequent staining of gels after separation. Gel images were captured by a Bio-Doc-It Imaging System. RNA was subsequently 
transferred to zeta-probe (BioRad), washed in 2x SSC (20X SSC: $3 \mathrm{M} \mathrm{NaCl}, 0.3 \mathrm{M}$ NaCitrate $\mathrm{pH} 7.4$ ) and $1 \%$ SDS (prepared as a $10 \% \mathrm{w} / \mathrm{v}$ solution with powder from Fisher) for 4 or more hours at $55^{\circ} \mathrm{C}$ and prehybridized overnight at $37^{\circ}$ in Ambion ULTRAhyb oligo hybridization buffer. Membranes were hybridized overnight with radiolabeled probes shown in Table 1. Membranes were subsequently washed with $6 \mathrm{XSSC}$ at $37^{\circ}$ and placed in phosphorimager cassettes for image capture using typhoon imaging software and band intensity quantified using Imagequant software (GE Healthcare). 


\begin{tabular}{|c|c|c|c|}
\hline $\begin{array}{l}\text { Probe } \\
\text { name }\end{array}$ & $\begin{array}{l}\text { Hybridization } \\
\text { location }\end{array}$ & $\begin{array}{l}\text { RP mutation and } \\
\text { Processing Defect } \\
\text { (Citation) }\end{array}$ & $\begin{array}{c}\text { Sequence } \\
\text { (H=human, } M=\text { mouse) }\end{array}$ \\
\hline 18SE & $\begin{array}{l}5 ' 18 S- \\
\text { ITS1 }\end{array}$ & $\begin{array}{l}\text { RPS19 } \\
\text { increased 21S (Flygare et } \\
\text { al., 2007) }\end{array}$ & $\begin{array}{l}\text { M: 5' CAG GCC GCC GCT CCT CCA } \\
\text { CAG TCT C 3' } \\
\text { H: 5' CCT CGC CCT CCG GGC TCC } \\
\text { GTT AAT GAT C 3' }\end{array}$ \\
\hline $\begin{array}{l}5.8 S- \\
\text { ITS2 }\end{array}$ & $\begin{array}{l}\text { 3'5.8S- } \\
\text { ITS2 5' }\end{array}$ & $\begin{array}{l}\text { RPL5, RPL11 } \\
\text { increased } 32 \text { S and 12S } \\
\text { (Rouquette et al., 2005) }\end{array}$ & $\begin{array}{l}\text { M: 5' GAC GCG ATT GAT CGT CAA } \\
\text { CCG ACG CT 3' } \\
\text { H: 5' GGG GCG ATT GAT CGG CAA } \\
\text { GCG ACG C 3' }\end{array}$ \\
\hline ITS2b & ITS2 & $\begin{array}{l}\text { RPL5, RPL11 } \\
\text { increased } 32 S \text { and } 12 S \\
\text { (Gazda et al., 2008) }\end{array}$ & $\begin{array}{l}\text { M: 5' CTG CGA CGC AAC TCC CAG } \\
\text { CCG CGC A 3' } \\
\text { H: 5' CTG CGA GGG AAC CCC CAG } \\
\text { CCG CGC A 3' }\end{array}$ \\
\hline ITS2 d/e & ITS2 & $\begin{array}{l}\text { RPL5, RPL11 } \\
\text { increased } 32 S \text { and } 12 S \\
\text { (Gazda et al., 2008) }\end{array}$ & $\begin{array}{l}\text { H 5' GCG CGA CGG ACG ACA CCG } \\
\text { CGG 3' }\end{array}$ \\
\hline $5 S$ & $5 S$ & $\begin{array}{l}\text { Mature rRNA } \\
\text { (Choesmel et al., 2007; } \\
\text { Rouquette et al., 2005) }\end{array}$ & $\begin{array}{l}\text { H: 5' CAG ACG AGA TCG GGC GCG } \\
\text { TTC 3' } \\
\text { M: Used human probe }\end{array}$ \\
\hline $5.8 \mathrm{~S}$ & $5.8 \mathrm{~S}$ & $\begin{array}{l}\text { Mature rRNA } \\
\text { (Choesmel et al., 2007; } \\
\text { Rouquette et al., 2005) }\end{array}$ & $\begin{array}{l}\text { H: 5' CAA TGT GTC CTG CAA TTC AC } \\
\text { 3' } \\
\text { M: Used human probe }\end{array}$ \\
\hline $18 S$ & $18 S$ & $\begin{array}{l}\text { Mature rRNA } \\
\text { (Choesmel et al., 2007; } \\
\text { Rouquette et al., 2005) }\end{array}$ & $\begin{array}{l}\text { H: 5' GCA TGG CTT AAT CTT TGA } \\
\text { GAC AAG CA 3' } \\
\text { M: used human probe }\end{array}$ \\
\hline
\end{tabular}

Table 1: Northern blot probes used for the identification of pre-rRNA processing intermediates and mature rRNAs 


\section{$\underline{\text { Cell Culture and Cytokines }}$}

All mammalian cell lines were cultured at $37^{\circ} \mathrm{C}$ with $5 \% \mathrm{CO}_{2}$ in a humidified atmosphere.

\section{TF-1 DBA Model}

The TF-1 erythroleukemic cell lines were maintained in RPMI 1640 (Mediatech) supplemented with 10\% heat inactivated fetal bovine serum (FBS), (Mediatech), 1\% P/S, (Invitrogen) and $5 \mathrm{ng} / \mathrm{ml} \mathrm{GM-CSF} \mathrm{(Peprotech).} \mathrm{The} \mathrm{TF-1}$ cell line with a doxycycline inducible shRNA targeting RPS19 mRNA was developed in the Karlsson laboratory as previously described (Miyake et al., 2005). Doxycycline (Sigma Aldrich) was added to cultures at a final concentration of $5 \mathrm{ng} / \mathrm{ml}$ for four to seven days before cells were harvested for subsequent experiments.

\section{$\underline{T F-1 \text { SBDS Model }}$}

The TF-1 erythroleukemic cell lines were maintained in RPMI 1640 (Mediatech) supplemented with 10\% heat inactivated fetal bovine serum (Mediatech), FBS, 1\% penicillin/streptomycin, P/S, (Invitrogen) and $5 \mathrm{ng} / \mathrm{ml}$ GMCSF (Peprotech). TF-1 cell lines transduced with lentiviral vectors (Sigma Aldrich) targeting SBDS mRNA were developed by Gulay Sezgin in the laboratory of Dr. Johnson Liu. Briefly, TF-1 cells were transduced at a cell density of $1 \times 10^{6}$ cells with lentiviral particles $\left[\left(200 \mu\right.\right.$ of $\left.10^{7} \mathrm{TU} / \mathrm{ml}\right)$ purchased from Sigma Aldrich] using $6 \mu \mathrm{g} / \mathrm{ml}$ polybrene. 
Sequences included in the lentiviruses targeting SBDS are:

\#1: 5' - CCGGGCCAAATACTTGCTTAAACTACTCGAGTAGTTTAAGCAAGTAT TTGGCTTTTTG - 3'

\#2: 5' - CCGGGCCAACAGTTAGAAATCGTATCTCGAGATACGATTTCTAACTG TTGGCTTTTTG - 3'

To establish stable cell lines, transduced TF-1 cells were placed under puromycin selection at $5 \mu \mathrm{g} / \mathrm{ml}$ (Fisher). Multiple clones for each of the two shRNA sequences used to reduce SBDS expression were established.

\section{Mouse Embryonic Stem Cell Models of DBA}

Mouse embryonic stem cell ( $m E S$ ) lines where one of two known DBA genes were inactivated by gene trap technology were purchased commercially. Two sets of cell lines with haploinsufficiency for Rps19 were obtained from the Mutant Mouse Regional Resource (MMRR) and one cell line mimicking haploinsufficiency for $R p / 5$ was obtained from the German Gene Trap

Consortium. A parental control cell line was also obtained for each gene trap. The S17-10H1 cell line was generated from the AK7.1 mouse embryonic stem cell parental line by electroporation of the ROSAFRY gene trap retroviral construct in intron 2 of a single copy of Rps19 (Fig. 3). A "rescue vector" to restore Rps19 protein expression was generated by inserting the Rps19 cDNA sequence into the pCMV6-A-Puro expression vector (Origene). This rescue plasmid called ORF-S17 was electroporated into the mES cells (Bio-Rad Gene 
Pulser). An empty vector control cell line was created in the same fashion. The cell line heterozygous for Rps19 transfected with the empty vector is called "EMT-S17." This cell line should mimic Rps19 haploinsufficiency observed in DBA patients. Cells with the rescue vector "ORF-S17" should mimic healthy controls. Both cell lines express a gene for puromycin resistance. 
Soriano retroviral gene trap

vector ROSAFARY*

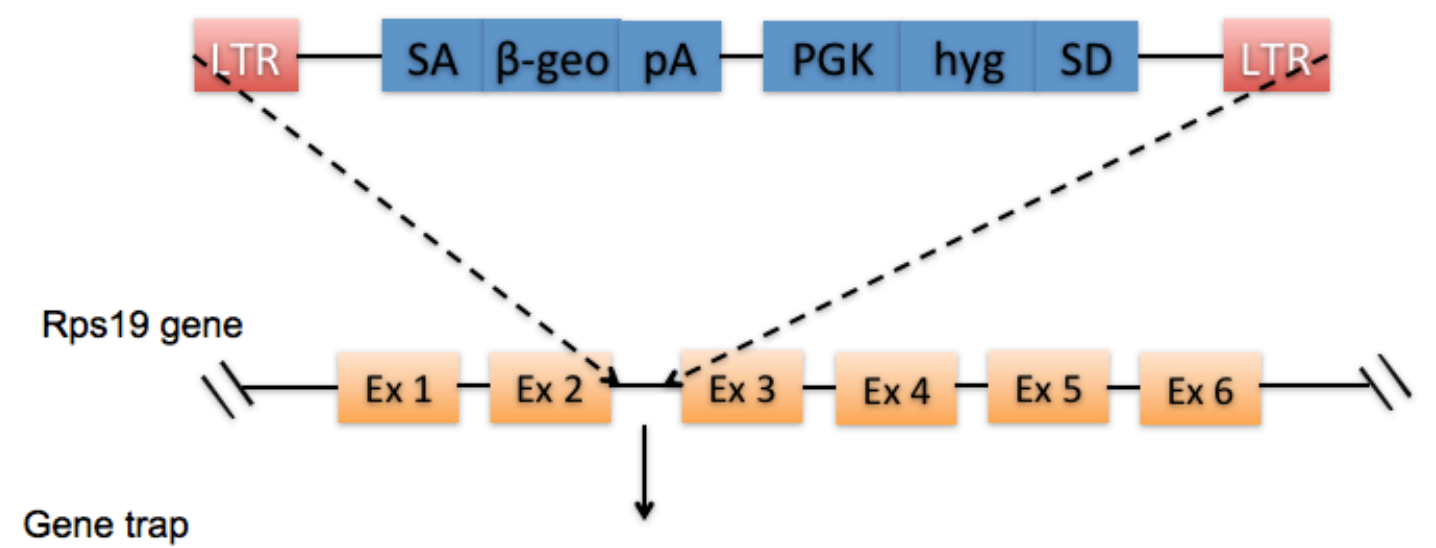
insertion "S17"

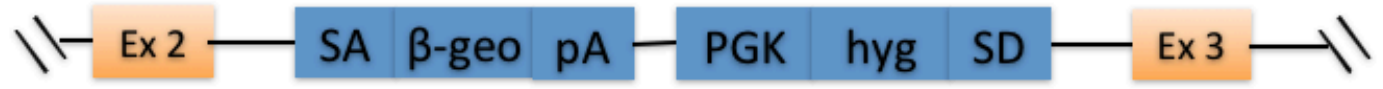

mRNA

\begin{tabular}{lllll|l|l|l} 
Ex 1 & Ex 2 & $\beta$-geo & hyg & Ex 3 & Ex 4 & Ex 5 & Ex 6
\end{tabular}

Fig. 3: Insertion of the ROSAFARY gene trap vector between exon 2 and exon 3 of Rps19 gene created the S17 gene trap cell line.

The ROSAFARY gene trap vector was used to create a genetrap library that has one gene in each cell line that is interrupted by this retrovirus. The AK7 mouse parental line has the inserted retroviral sequence located between exons two and three of the Rps19 gene, leading to two fusion genes. The first fusion gene is referred to as a promoter trap and creates a fusion gene between the gene being disrupted and the $\beta$-geo gene coding for neomycin resistance. Transcription is halted downstream of $\beta$-geo by a poly $(A)$ addition sequence. Since this fusion gene expresses only exons 1 and 2 of Rps19 it is unlikely to lead to a functional 
product. The second fusion gene links the hyg gene, a selection marker, to exons 3-6 of Rps19. Again, this fusion gene is unlikely to produce a functional Rps19 protein. 
The second Rps19 gene trap model, termed YHC074, was also purchased from MMRC and was generated using the parental mES cell line E14Tg2a.4. The pGTOlxf gene trap vector in this cell line is inserted into intron 3 , between exon 3 and 4 of the Rps19 gene (Fig. 4). The third mES gene trap cell line, D050B12, was purchased from the German Gene Trap Consortium and was generated from the parental TBV-2 mES cell line. This cell line was created using the ROSAFRY gene trap vector inserted into intron 3 of the Rp/5 gene (Fig. $4)$. 


\section{Rps19 gene \\ $\downarrow \operatorname{Ex} 1-\operatorname{Ex} 2-\operatorname{Ex} 3-\operatorname{Ex} 4-\operatorname{Ex} 5-\operatorname{Ex} 6-\backslash$}

Gene trap insertion "S17"

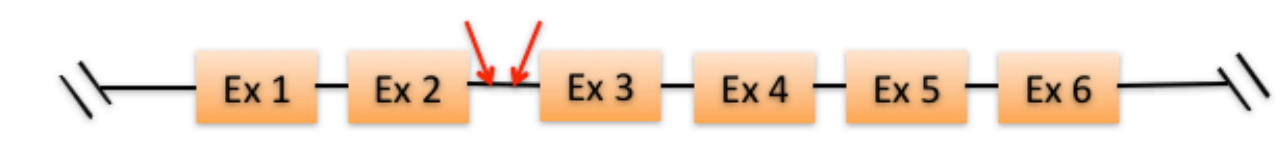

Gene trap insertion "YHC074"

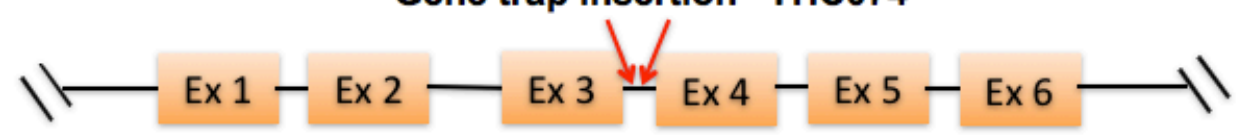

Rpl5 gene
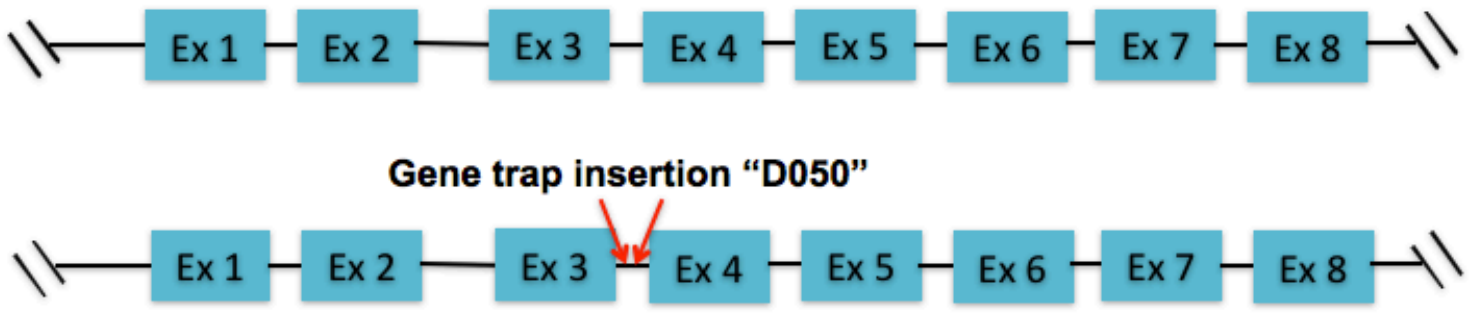

Fig. 4: Location of gene trap insertions in mES cell lines used.

The S17 cell line contains the insertion between exons two and three of Rps19.

The YHC074 cell line contains a gene trap insertion in between exons three and four of Rps19. The D050 cell line contains a gene trap insertion in between exons three and four of the Rp/5 gene. (Gene trap technology is described in greater detail in the legend of Fig. 3). 
Mouse embryonic stem cells were cultured under the conditions established by stem cell technologies ( $\underline{w w w . s t e m c e l l . c o m})$. Tissue culture dishes were coated by adding an excess of $0.1 \%$ gelatin solution from Stem Cell Technologies ( $3 \mathrm{~mL}$ for a T25 flask, with proportional increases depending on dish size), allowing the plate to sit at room temperature in the biosafety cabinet for 20 minutes, removing excess gelatin, and allowing the plates to dry before use. Maintenance media was prepared using DMEM high glucose (Invitrogen) supplemented with $15 \%$ stem cell grade FBS (Stem Cell Technologies), $100 \mu \mathrm{M}$ non-essential amino acids (Stem Cell Technologies), $100 \mathrm{U} / \mathrm{ml}$ penicillin, $10 \mu \mathrm{g} /$ $\mathrm{ml}$ streptomycin (Invitrogen), $2 \mathrm{mM}$ glutamine (Glutamax @Invitrogen) $100 \mu \mathrm{M}$ monothioglycerol (MTG; Sigma), and $10 \mathrm{ng} / \mathrm{ml}$ mouse leukemia inhibitory factor (mLIF; Stem Cell Technologies). A stock solution of mLIF was made fresh on the day of media preparation by diluting 1:100 in DMEM and adding $43 \mu \mathrm{L}$ to each 50 $\mathrm{ml}$ aliquot of media. Media was sterile filtered prior to addition of $\mathrm{mLIF}$ and was kept for no more than one week at $4^{\circ} \mathrm{C}$. The TBV-2 and its derived line, D050B12, were originally maintained on an embryonic fibroblast feeder layer (Stem Cell Technologies) in the presence of mitomycin C (Sigma) before being plated on gelatin coated plates without fibroblasts to develop feeder independent cell lines. All experiments were performed on feeder independent cell lines. The other two sets of mES cells were always plated without feeders on gelatin coated tissue culture dishes. 


\section{Animal Models}

\section{Inducible Rps19 knockdown}

All animals were maintained at Lund University, Sweden by the laboratory of Stefan Karlsson. Tssues and cell lines were shipped to the University of Louisville for further study. Briefly, embryos were developed with one or two copies of either sequence "B" or sequence "D" shRNA designed to target Rps19 mRNA under the control of a tet-on promoter located in the collagen A1 locus (Jaako et al., 2011). Animals with single copies of the inducible shRNA (B/+ or D/

+) were termed "single dox," and those with two copies of the inducible shRNA (B/B or $\mathrm{D} / \mathrm{D}$ ) were demarcated as "double dox." Liver and bone marrow samples from mice given doxycycline in their drinking water for two weeks were sacrificed and used for the experiments outlined below.

Mouse embryonic fibroblasts (MEF) from the Karlsson mice were maintained in DMEM media (Mediatech), supplemented with 10\% FBS (Mediatech), and 1\% P/S (Invitrogen). To decrease Rps19 expression, doxycycline (Sigma Aldrich) was added to a final concentration of $1 \mu \mathrm{g} / \mathrm{ml}$ or 5 $\mu \mathrm{g} / \mathrm{ml}$ for seven to fourteen days before sample harvest (Jaako et al., 2011).

\section{Cell Viability and Rescue with NAC}

Cell counts were performed by taking an aliquot of cells and diluting with an equal volume of Trypan Blue (MP Biomedicals) and adding $10 \mu \mathrm{L}$ of the resulting solution to a hemocytometer (Hausser Scientific) to determine cell 
number. All viability experiments were performed in triplicate with three separate counts obtained for each experiment.

After four days of growth, the cell cultures were treated with various concentrations of $\mathrm{N}$-acetyl cysteine (Sigma). The stock solution of $500 \mathrm{mM}$ was prepared in RPMI media and dilutions made to a final concentration of $50 \mu \mathrm{M}$, $100 \mu \mathrm{M}, 500 \mu \mathrm{M}$, and $1 \mathrm{mM}$ when added to cultures at day four. After 24 hours of treatment the cultures were counted as described above and used for cell cycle experiments as delineated in the flow cytometry section.

\section{Polysome Profiling}

Cells grown in culture were treated with a $1 / 100$ vol of $9 \mathrm{mg} / \mathrm{ml}$ cycloheximide (Sigma) for 10 minutes at $37^{\circ} \mathrm{C}$. All solutions used in harvesting polysome samples In subsequent steps also contianed the same concentration of cycloheximide. Cells were trypsinized ( $0.25 \%$ Trypsin EDTA, Invitrogen) when appropriate, or pelleted by centrifugation at $800 \times g$ for 5 minutes. Cells were washed with cold PBS (VWR), and lysed using a handheld homogenizer (Fisher) in polysome buffer containing 50 mM Tris-HCl pH 7.5 (Fisher BP152-1, prepared with DEPC treated water as a $1 \mathrm{M}$ stock solution and titrated to $\mathrm{pH} 7.5$ with concentrated $\mathrm{HCl}$ ), $240 \mathrm{mM} \mathrm{NaCl}$ (Fisher S271-1 prepared with DEPC treated water as a stock solution of $2 \mathrm{M}$ ), $10 \mathrm{mM} \mathrm{MgCl} 2$ (Sigma M1028), $5 \mathrm{mM} \beta$ mercaptoethanol (Sigma), 250 mM Sucrose (Fisher BP220-212), 2\% Triton X (Sigma), $100 \mu \mathrm{g} / \mathrm{ml}$ heparin (Alfa Aesar), and $90 \mu \mathrm{g} / \mathrm{ml}$ cyclohexamide (SigmaC7698). Debris was pelleted in a refrigerated microcentrifuge (Eppendorf) at 
$8000 \times g$ for 10 minutes and the RNA concentration of the supernatant determined using a Nanodrop (Thermo Scientific). Equal quantities of total RNA in a maximum volume of $350 \mu \mathrm{L}$ were added to the top of $15-55 \%$ sucrose gradients. Gradients were poured using a bone marrow biopsy needle into RNAse/DNAse free round bottom polyallomer $17 \mathrm{~mL}$ tubes (\#5042) from Seton Scientific with $3 \mathrm{~mL}$ layers of $15 \%(\mathrm{w} / \mathrm{v}), 25 \%, 35 \%, 45 \%$, and $55 \%$ sucrose (Fisher BP220-212) containing 25 mM pH 7.5 Tris-HCl (Fisher: BP152-1, prepared with DEPC treated water as a $1 \mathrm{M}$ stock solution and titrated to $\mathrm{pH} 7.5$ with concentrated $\mathrm{HCL}) 25 \mathrm{mM} \mathrm{NaCl}$ (Fisher S271-1 prepared with DEPC treated water as a stock solution of $2 \mathrm{M}$ ) and $5 \mathrm{mM} \mathrm{MgCl}_{2}$ (Sigma M1028, stock solution). Gradients were centrifuged at $28,000 \mathrm{rpm}$ in a SW 28 rotor for $7-12$ hours using a Beckman L8-M ultracentrifuge. The gradient fractions were then collected using an ISCO density gradient fractionator (Model 185), retriever (Retriever 500), and flourinert (Sigma) and visualized using a UA-6 UV/Vis detector (ISCO), and type 11 optical unit with filters corresponding to $254 \mathrm{~nm}$ (ISCO) (Jaako et al., 2011).

\section{Western Blotting}

Cell pellets were harvested and lysed by snap freezing in RIPA buffer containing leupeptin, aprotinin, PMSF, and protease inhibitor cocktail (Sigma). Cell debris were pelleted by centrifugation at $5,000 \times g$ in a refrigerated microcentrifuge (Eppendorf). Protein concentration was determined using the BCA method (Thermo Fisher) and equal concentrations of protein were suspended in Nupage buffer (Invitrogen) containing dithiothreitol ( 50mM DTT; 
Sigma). SDS-PAGE was performed using $2.5 \mu \mathrm{g}$ to $10 \mu \mathrm{g}$ of protein and $4-12 \%$ Bis-Tris Ready gels (Invitrogen) with protein size estimated by Biorad dual color standards. After transferring proteins to nitrocellulose membranes (BioRad) in transfer buffer containing 20\% methanol (Fisher) overnight at a constant 20 Volts at $4^{\circ} \mathrm{C}$ (BioRad transfer apparatus), membranes were blocked with $5 \%$ milk in TBST (Fisher) and immunoblotting was performed using the following antibodies: anti-RPS19 (H00006223-M01, Abnova; 1:1,000), anti-RPL5 (ab74744, Abcam; 1:500-1:1000), anti-p53 (9282, Cell Signaling; 1:1000-1:2000), anti-RNA Polymerase II p.14.5 (abs8436, Abcam; 1:2,000), anti-LDH-A (SC27230, Santa Cruz; 1:1,000), anti-VDAC1 (4661, Cell Signaling; 1:1,000), anti-cytochrome c (SC-7159 Santa Cruz; 1:1,000), anti-Tom20 (a custom antibodyreceived as a generous gift from Dr. Brian Wattenberg1:500), anti-pyruvate dehydrogenase, PDH, (SC-65242, Santa Cruz, 1:1,000), anti-superoxide dismutase 2, SOD2, mitochondrial (SC-20080, Santa Cruz, 1:1,000), anti-Tom40 (SC-11414, Santa Cruz, 1:1,000), anti-isocitrate dehydrogenase 3a, IDH3 $\alpha$, (AP1927a, Abgent, 1:1,000), anti-IDH2 (H00003418-A01, Novus biological supplied by Abnova, 1:1,000), anti- $\beta$ tubulin (ab6046, Abcam, 1:1,000) and anti- $\beta$-actin (SC69879 AC-15 Santa Cruz; 1:5,000). Secondary antibodies used were either goat antimouse (\#3430 Thermo Scientific and Santa Cruz), goat anti-rabbit (7074, Cell signaling technology), or donkey anit-goat (SC2020, Santa Cruz) as dictated by the primary antibody. The resulting complexes were detected using chemiluminescent reagents and protocols from Pierce Chemical, GE Healthcare, or Thermo Scientific. 


\section{Flow cytometry}

\section{Cell Cycle Analysis}

To analyze the relative amounts of cells in different phases of the cell cycle in asychronized cultures, $1 \times 10^{6} \mathrm{TF}-1$ cells expressing lentiviral vectors targeting SBDS mRNA or scrambled controls were harvested and washed with PBS. Cells were fixed in $70 \%$ ethanol for at least $30 \mathrm{~min}$ at $-20^{\circ} \mathrm{C}$ and washed twice with PBS. After resuspension in $1 \mathrm{ml}$ of PBS, cells were treated with ribonuclease at $100 \mu \mathrm{g} / \mathrm{ml}$ (RNAse A, Qiagen, MD). Propidium iodide was added to a final concentration of $50 \mu \mathrm{g} / \mathrm{ml}$ (Invitrogen), and samples were analyzed with a BD FACS Calibur (BD Biosciences, San Jose, CA) cytometer. The events were gated to remove both debris and doublets (signals generated by clumps of two or more cells) and 20,00-50,000 total events were used for subsequent analyses. The FL3H-PI channel was used for data collection. The resulting histograms were analyzed using FlowJo software and the Dean/Jett/Fox method used to quantify phases of the cell cycle (Treestar Ashland, OR) (Dempster A, 1977; Fox, 1980; Krishan, 1975; S, 1997; Shapiro, 2003; Watson et al., 1987; Webb, 2002).

\section{JC-1 Mitochondrial Membrane Potential Assay}

Mitochondrial membrane potential was assessed using the JC-1 Assay kit from Molecular Probes according to the manufacturer's instructions. This assay measures mitochondrial membrane potential irrespective of mitochondrial size or density. The JC-1 compound ((5,5',6,6'-tetrachloro-1,1',3,3'-tetraethylbenzimidazolylcarbocyanine iodide) shows a shift in emission spectrum from green (529 
$\mathrm{nm}$ ) when the dye is outside of mitochondria to red $(590 \mathrm{~nm})$ as the dye enters mitochondria in a membrane potential-dependent manner and forms aggregates that are no longer able to leave the mitochondria. Pretreatment with $50 \mathrm{mM}$ CCCP, an uncoupler which dissipates the proton gradient, prevents the accumulation of $\mathrm{JC}-1$ in mitochondria and served as a negative control. Unstained cells served as a control for cellular autofluorescence. After incubation with the $\mathrm{JC}-1$ compound for 20 minutes at $37^{\circ} \mathrm{C}$, cells were harvested and suspended in PBS for analysis with a BD FACS Calibur (BD Biosciences) cytometer. A dot plot of side scatter (SSC) and forward scatter (FSC) was used to determine the gating of the appropriate population of live cells and to remove debris. At this point any extracellular JC-1 that wasn't removed as cells were harvested and washed was also eliminated.

The following strategy was employed to analyze the membrane potential data generated by flow cytometry. The FL2 channel was used to measure red fluorescence and is graphically represented on the y-axis and the FL-1 channel was used to measure green fluorescence which is graphed on the x-axis. Quadrants were created in the plot to capture different cell populations. The lower left quadrant represents auto-fluorescence and was defined by unstained control samples. The upper right quadrant includes cells expressing high levels of green and red fluorescence and represents the mitochondrial potential of control TF-1 cells. The lower right quadrant represents cells with relatively high green fluorescence but low red fluorescence as would be expected if the membrane potential was decreased (Chaoui et al., 2006; Chazotte, 2011; 
Cossarizza and Salvioli, 2001; Garner and Thomas, 1999; Guthrie and Welch, 2008; Keil et al., 2011; Legrand et al., 2001; Lugli et al., 2007; Reers et al., 1995; Salvioli et al., 1997; Simeonova et al., 2004; Smiley et al., 1991). A minimum of 40,000 events were collected for each sample replicate. Analyses were performed using FlowJo software from Treestar to perform appropriate compensation, gating, and comparison of all replicate data using the same parameters.

\section{Annexin V/ Propidium lodide Assay of Apoptosis}

Flow cytometry was also employed to study apoptosis in our cell lines. Cells were stained with annexin and propidium iodide $(\mathrm{PI})$ prior to evaluation by flow cytometry. An Annexin V-FITC/PI kit was purchased from E-Biosciences and experiments performed according to the manufacturer's instructions. The annexin $\mathrm{V}$ stain recognizes outer membrane phosphatidylserine molecules whose hydrophilic head flips from the inner to the outer membrane during early phases of apoptosis. Co-staining with PI allows one to exclude cells from the analysis whose membranes are no longer intact, as PI stains the DNA/RNA species of necrotic cells or those in the later stages of apoptosis. The samples were analyzed using the BD FACS Calibur (BD Biosciences) with a minimum of 40,000 events collected. The samples were gated using an unstained control sample as well as single-stained controls treated with only PI or annexin-V. The data collected were then analyzed using FlowJo software (Treestar). Data were

plotted with FL3-PI on the Y-axis and FL1-H: annexin $\mathrm{V}$ on the $\mathrm{X}$ axis. As before, 
the lower left quadrant represents autofluorescence, as was delineated using unstained control samples. Cells staining highly for both $\mathrm{PI}$ and Annexin-V were then found in the top right corner. These samples were labeled as "late apoptosis." Those samples contained in the lower right corner, staining highly for Annexin-V only were considered "early apoptotic" cells. The percentage of events contained within each of the quadrants was then calculated.

\section{$\underline{\text { Reactive oxygen species }}$}

Reactive oxygen species were measured using 2',7'-dichlorfluoresceindiacetate (DCFH-DA). DCFH-DA is rapidly converted to the non-fluorescent compound DCFH once inside cells by cellular esterases. In the presence of ROS cellular peroxidases convert DCFH to DCF which is highly fluorescent. Measurement of DCF fluorescence thereby provides a measure of cellular ROS. This assay for detecting ROS was modified from previously published work (Ambekar et al., 2010). Viable TF-1 cells were counted using Trypan blue exclusion and plated in 96 well plates (Costar) at a density of $1 \times 10^{5}$ cells/well. A minimum of three replicates was used for each sample including control treated with DMSO and CCCP. DCFH-DA (Sigma) suspended in DMSO (Sigma) was added to wells to a final concentration of $20 \mu \mathrm{M}$. After incubation at $37^{\circ}$ for 15 minutes the plates were then read once every minute using filters appropriate for an excitation wavelength of $505 \mathrm{~nm}$ and emission at $529 \mathrm{~nm}$ using the Synergy plate reader (Biotek). After subtracting the intensity of the blank (DMSO), values 
for three replicate experiments are represented as a ratio of fluorescence intensity with SBDS knockdown relative to scrambled controls.

\section{Oxygen consumption}

Oxygen consumption was measured using a Gilson oxygraph with a Clark-type electrode following previously published protocols (Campian et al., 2007; Gao et al., 2010). Cells were suspended in $550 \mu \mathrm{l}$ of growth media at a concentration of $2.7 \times 10^{6} \mathrm{cell} / \mathrm{ml}$ and placed in the chamber. Basal respiration tracings of oxygen consumption were monitored for two minutes. Carbonyl cyanide $m$-chlorophenyl hydrazine, CCCP (Sigma), was added to the chamber using a Hamilton Syringe to a final concentration of $10 \mathrm{mM}$ to measure maximal respiration rates of each sample for eight additional minutes. Respiration rates were expressed as $\mu \mathrm{mol}$ of oxygen consumed per million cells per minute. Linear portions of the curves were then used to express respiration rates in terms of the basal respiration rate relative to the maximal respiration rate.

\section{Statistical Analysis}

All data are presented as the result of no less than three independent experiments. Microsoft's Excel program and Graphpad's Prism software were used to perform all statistical analyses of replicate data. When appropriate an unpaired Student's t-test was used to determine the significance of differences between sample means. ANOVA statistical analyses were run when indicated by sample demographics and the appropriate post-hoc tests used based on sample 
parameters. Unless otherwise stated, bar graphs represent the mean of all experimental replicates using the standard deviation for error bars and asterisks represent $p$-values as such: ${ }^{*} p<0.05,{ }^{* *} p \leq 0.01,{ }^{* * *} p \leq 0.005$. 


\title{
CHAPTER III
}

\section{THE VITAL ROLE OF PRE-RRNA PROCESSING IN DBA DIAGNOSIS AND GENE DISCOVERY}

\author{
$\underline{\text { Introduction }}$
}

Diamond Blackfan Anemia, (DBA), was first linked to a mutation in the gene encoding a ribosomal protein, RPS19, in 1999. Since then, causative mutations have been indentified in 10 genes that encode protein components of both small and large ribosomal subunits (Ball, 2011; Draptchinskaia et al., 1999). Despite this progress, the genes affected in many patients have not been identified using DNA sequence analysis of candidate ribosomal protein genes. Current genome-wide efforts have been able to identify patients with large deletions including known ribosomal protein genes that were not picked up by DNA sequencing. Still, the underlying genetic defect is still unknown in approximately $40 \%$ of DBA patients (Boria et al., 2010; Farrar et al., 2011).

Pre-rRNA processing experiments have played a critical role in gene discovery efforts in DBA, linking mutations in prospective genes to defects in ribosome synthesis (Campagnoli et al., 2008; Choesmel et al., 2008; Farrar et al., 2008; Flygare et al., 2007; Robledo et al., 2008; Rouquette et al., 2005). The role of pre-rRNA processing in gene discovery has become even more critical as 
whole genome approaches have been applied to identifying new DBA genes whose potential role in ribosome synthesis is potentially more tenuous than that for ribosomal protein genes. The work described in this chapter illustrates the contribution pre-RNA processing studies can make to diagnosing DBA in challenging clinical cases and in discovering new genes beginning with the description of a particularly challenging case. An outgrowth of these studies is the identification of a new strategy for tracking pre-rRNA processing defects associated with genes encoding large subunit ribosomal proteins. We feel that it should be possible for clinical laboratories to develop this approach as a new diagnostic tool for DBA. Finally, these studies highlight ribosomal proteins RPL5 and RPL11 as playing a unique role in DBA pathophysiology based on their interaction with 5S rRNA and the distinctive role of the RPL5/RPL11/5S rRNA complex both in ribosome biogenesis and signaling ribosome stress. 


\section{$\underline{\text { Case Report }}$}

A child presented to a physician in the San Diego area at four months of age with a severe macrocytic anemia. She also had neutropenia and elevated numbers of morphologically normal platelets, which does not fit the classical criteria for DBA. She could however potentially fall under a broader diagnostic heading of non-classical DNA. Non-classical DBA includes patients exhibiting effects on myeloid lineages other than erythrocytes (Boria et al., 2010; Farrar et al., 2011; Vlachos et al., 2008). In addition to her hematopathology, the proband had multiple congenital anomalies including bilateral fusion of the radius and ulna. Both of her thumbs showed aberrations in development that were not symmetrical. One hand contained a triphalangeal thumb and the other was hypoplastic. Bone marrow biopsy revealed a paucity of erythroid precursors and a block in maturation of granulocyte precursors that would normally differentiate into neutrophils, and a normal female (46XX) karyotype. Given the lack of a clear diagnosis based on the patient's clinical profile, molecular studies analyzing the possible involvement of multiple inherited bone marrow failure syndromes (IBMFS) were performed.

The litany of laboratory tests that ensued began with analyses of defects associated with the most common IBMFS, Fanconi Anemia (FA) (Shimamura and Alter, 2010). Fanconi anemia results from defects in DNA repair and patient lymphoblasts were tested for their sensitivity to DNA crosslinking agents, a routine test for this disease (Shimamura and Alter, 2010). These tests were negative. Shwachman Diamond syndrome was also considered unlikely as no 
pathogenic mutations were found in the SBDS gene, which is affected in over 90\% of SDS patients (Dror and Freedman, 1999, 2002; Johnson and Ellis, 2011; Nihrane et al., 2009; Tsangaris et al., 2011; Yamaguchi et al., 2011). Tests were also performed on the eleven ribosomal protein genes known to be involved in DBA. While two single nucleotide polymorphisms were found, one each in RPS19 and RPL11, these changes were unlikely to be deleterious.

Our collaborators then turned to comparative genomic hybridization $(\mathrm{CGH})$, a technique for scanning the entire genome for copy number variations. $\mathrm{CGH}$ on this patient revealed a 3.2 megabase deletion on chromosome 2 at 2q11.2. This result was confirmed by fluorescence in situ hybridization. Further, as neither parent exhibited this deletion it was presumed to have arisen spontaneously in the child. The deletion at 2q11.2 observed in this child includes a number of genes including RPL31, which encodes a ribosomal protein of the $60 S$ subunit. This suggested the child could be haploinsufficient for RPL31, which would be consistent with a diagnosis of Diamond Blackfan anemia. However, as RPL31 has not been identified as a DBA gene previously, we felt it was important to address whether the $2 \mathrm{q} 11.2$ deletion was associated with a defect in ribosome biogenesis in this patient.

To address whether this child had a defect in ribosome biogenesis, we employed a protocol we recently developed as part of a consortium of investigators studying the molecular basis of DBA. This protocol involves the isolation of mononuclear cells from peripheral blood, followed by culturing and activating T lymphocytes with concanavalin A (ConA) (Farrar et al., 2011). Total 
RNA isolated from these cells is then used for pre-rRNA processing studies. This technique has so far revealed robust and reproducible pre-rRNA processing defects in many DBA patients. 


\section{$\underline{\text { Results }}$}

\section{Pre-rRNA processing defects associated with haploinsufficiency for RPL31}

Fig. 5A shows an ethidium bromide stained RNA samples in a formaldehdye gel of samples obtained from the index proband from San Diego. The samples were sent to us blinded, and the codes revealed only after preRNA processing studies were completed and data analyzed. Samples included the proband, her unaffected parents, and an unrelated healthy control. Even at the level of ethidium bromide visualization with UV, it was clear one of the four samples stood out from the others. Sample 06518 has a prominent band above that of $28 \mathrm{~S}$ rRNA, later shown by hybridization to be $32 \mathrm{~S}$ pre-rRNA (Fig. 5B and Fig 6). Subsequent decoding of the samples revealed that 06518 , was in fact derived from the proband. As shown in Fig. 1, 32S pre-rRNA contains both $5.8 \mathrm{~S}$ and 28S mature RNAs separated by an intervening sequence labeled ITS2. Cleavages within ITS2 ultimately give rise to the $3^{\prime}$ end of 5.8 S RNA and the $5^{\prime}$ end of 28S RNA. The pre-RNA processing defect in this sample is specific for RNA components of the large subunit as would be expected for RPL31, a large subunit ribosomal protein. 

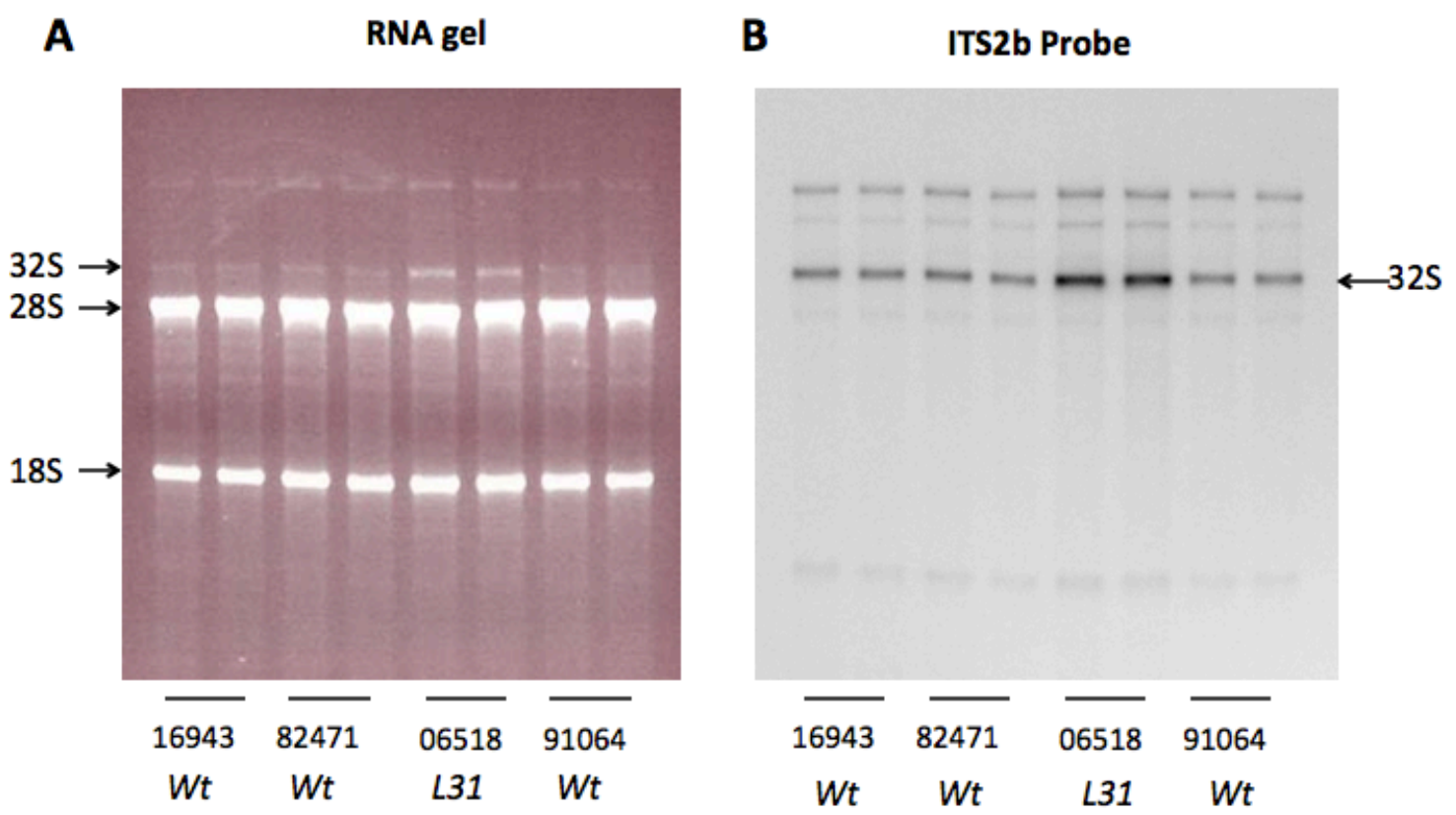

Fig 5: Pre-rRNA processing defect associated with haploinsufficiency for RPL31

Total RNA from blood mononuclear cells cultured for 96 hours in the presence of ConA was isolated and separated on $1.5 \%$ formaldehyde agarose gels. Sample codes and RPL31 gene status are listed below each duplicated sample. (A) RNA gel of ethidium bromide stained samples (B) RNA was transferred to zeta-probe and hybridized with probe ITS2b (Sequence in Table 1). The pre-rRNA labeled $32 S$ pre-rRNA was confirmed using a number of different oligonucleotides for hybridization to demarcate its boundaries in both this gel (Fig 6) and in a gel with other DBA patient samples shown in Fig 7 and 8. 
A

Probe: 5.8S- ITS2

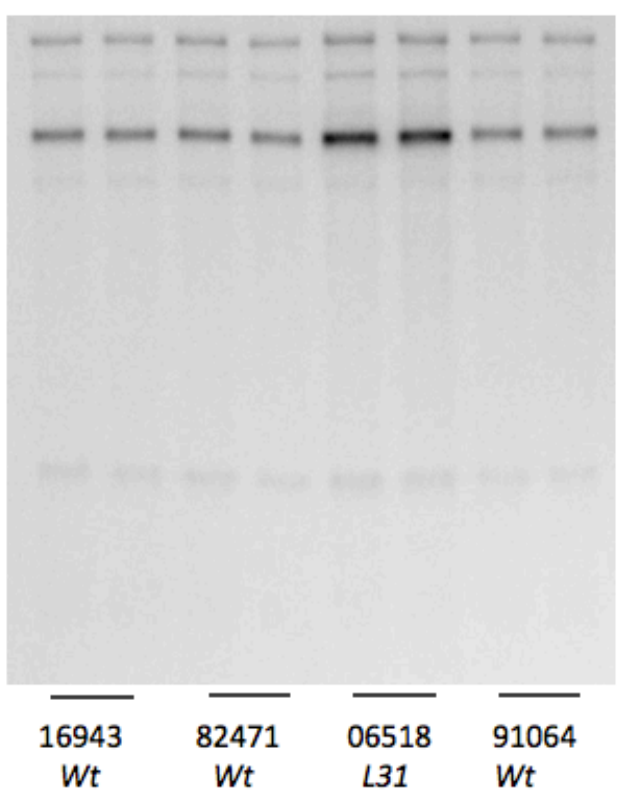

B

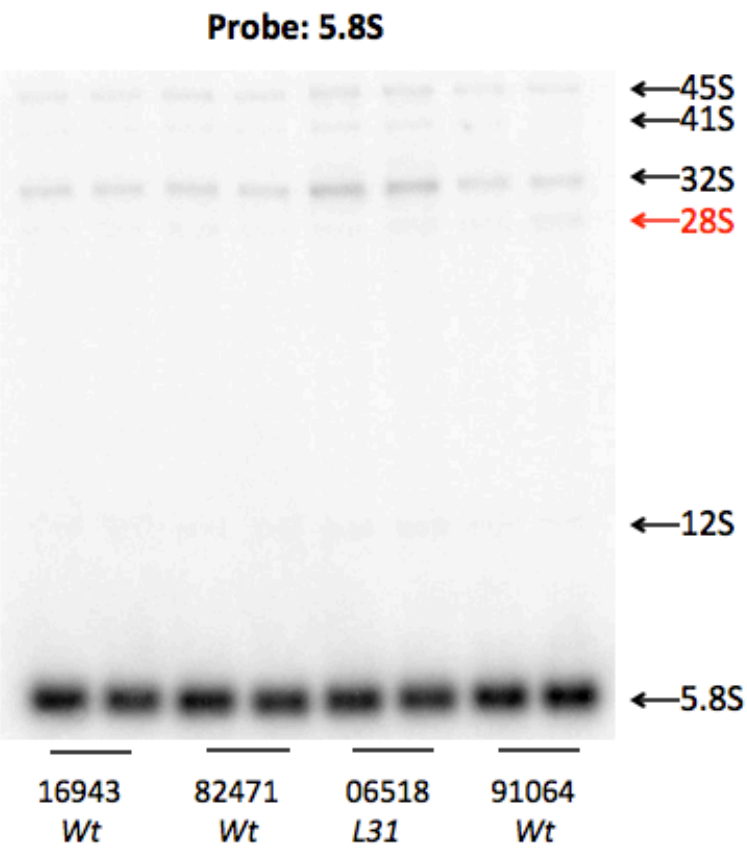

Fig 6: Northern blot of RNA isolated from the index proband, unaffected parents, and a healthy control reveals a processing defect in proband prerRNA

(A) Northern blot of the gel shown in Fig. 5 after probing with an oligonucleotide complementary to sequences bridging the $3^{\prime}$ end of $5.8 \mathrm{~S}$ rRNA and the $5^{\prime}$ end of ITS2. (B) The membrane in A was stripped and probed with an oligonucleotide complementary to mature 5.8S rRNA. The pre-rRNA and rRNA species are labeled to the right of the northern blots and the patient number and genotype shown below. All samples were run in duplicate. 
A common pre-rRNA processing signature in patients haploinsufficient for large

subunit ribosomal proteins

To compare the pre-RNA processing defect associated with RPL31 haploinsufficiency with that for haploinsufficiency of other large subunit ribosomal proteins, we analyzed samples from patients with mutations in either RPL5 or RPL35A, two known DBA genes encoding large subunit proteins (Farrar et al., 2008; Gazda et al., 2008). Fig. 7A shows an ethidium bromide-stained gel of the different patient samples together with an unaffected parent of the RPL31 patient. Here again, a prominent band just above the 28S rRNA is evident in the patient samples, but is present in much lower amounts in the healthy controls. Fig. 7B shows this band is $32 \mathrm{~S}$ pre-rRNA. Thus, it would appear from each of the DBA samples we have tested where a large subunit ribosomal protein gene is responsible for the disease, there is a clear increase in 32S pre-RNA evident at the level of ethidium bromide staining. A similar sized band has been observed in RNA gels of patients with mutations in RPL11 (Elisa Pavesi, University Eastern Piemonte, Novara Italy, personal communication) indicating that an increase in $32 S$ pre-rRNA may be a common characteristic of deficiencies of large subunit ribosomal proteins. Given that this increase can be visualized by gel electrophoresis and ethidium bromide staining, the increase in 32S pre-rRNA may be developed as a diagnostic tool for DBA when genes encoding large subunit ribosomal proteins are suspected. 


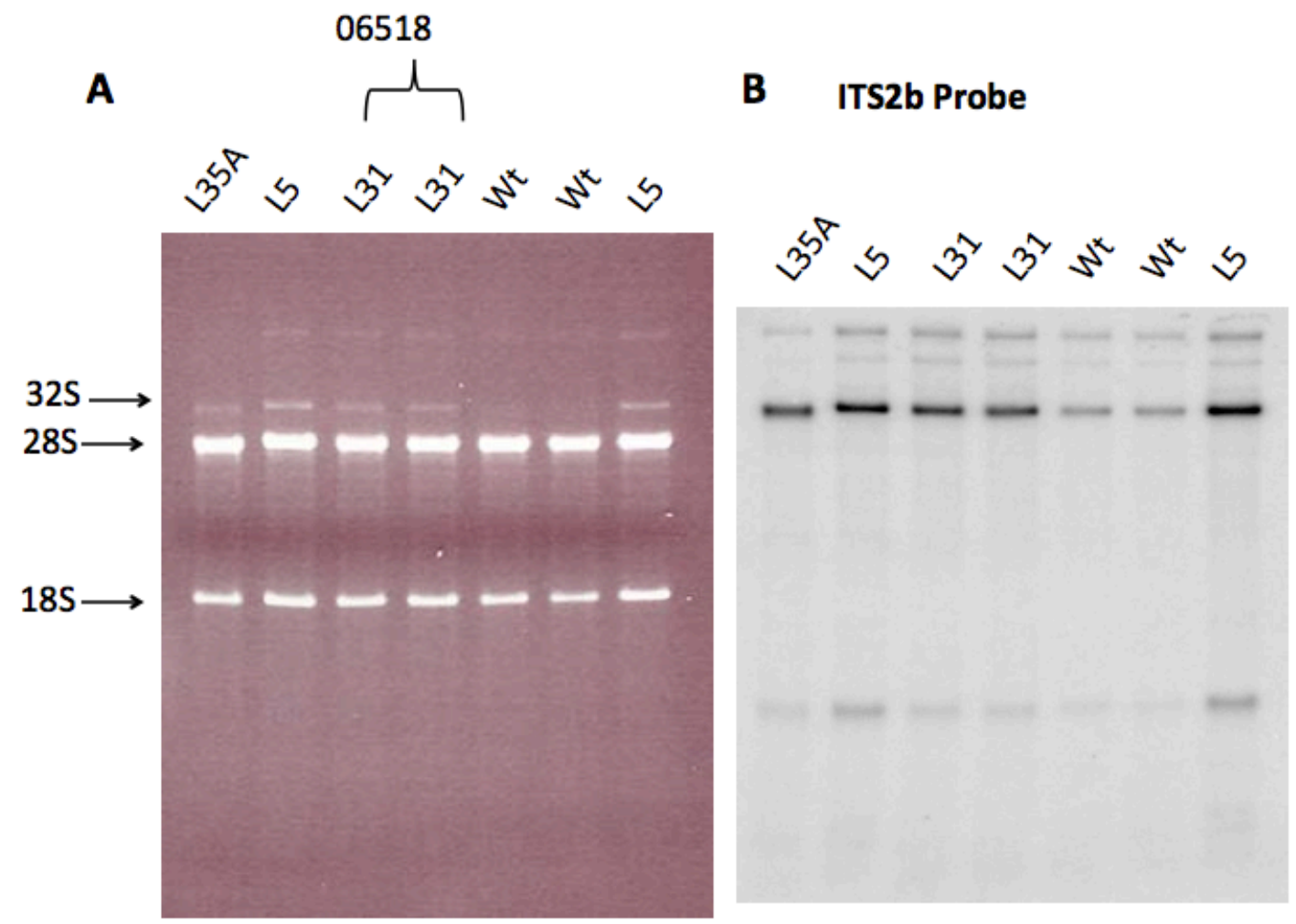

Fig 7: Evidence of a common pre-rRNA processing signature associated with haploinsufficiency for large subunit ribosomal proteins.

(A) Total RNA was prepared from peripheral mononuclear cells cultured for 96 hours in the presence of ConA. RNA was fractionated on $1.5 \%$ formaldehyde agarose gels and stained with ethidium bromide. Affected genes in patient samples are listed above each lane. When a gene is listed twice this denotes duplicated samples from the same patient. The healthy control is denoted as Wt. (B) RNA was transferred to a zeta-probe membrane and hybridized with oligonucleotide "ITS2b" which is complementary to sequences within ITS2 (sequences shown Table 1). 


\section{S RNA is selectively decreased in cells haploinsufficient for RPL5}

To determine if we could identify unique pre-rRNA processing characteristics associated with any of the large subunit protein genes studied here, we analyzed the membrane above (Fig. 7) using additional probes. The most striking difference between the pre-rRNA processing patterns for the affected genes is the decrease in 5S rRNA observed in the cells derived from a patient with mutated RPL5 (Fig. 8B). The decrease is selective for 5S RNA as there is little change in 5.8S RNA in the RPL5 sample compared to the others (Fig. $8 \mathrm{C}$ ). Thus, it may be possible to further refine the diagnostic power of prerRNA processing to identify signatures associated with specific ribosomal protein gene mutations. 


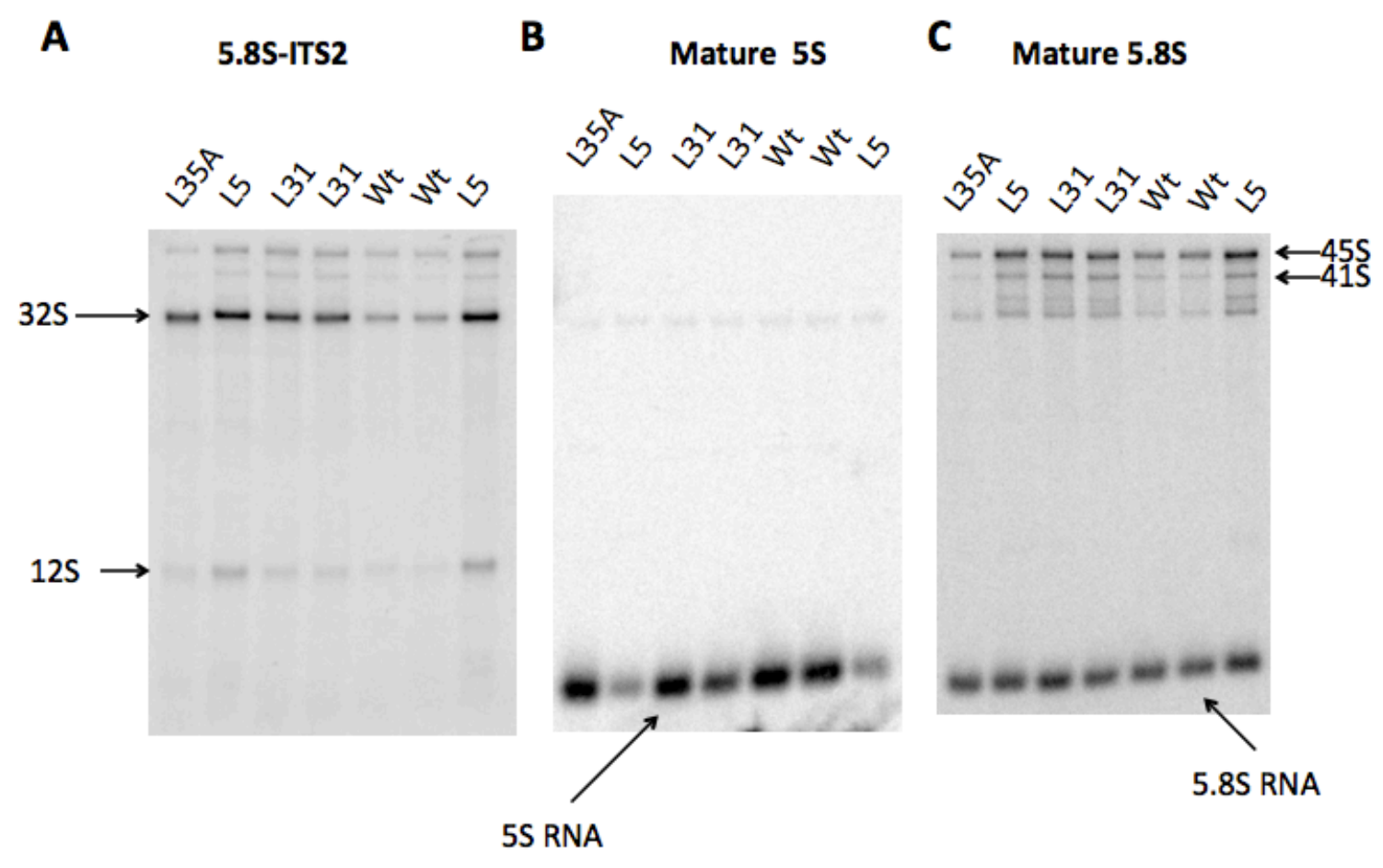

Fig 8: Northern Blots of DBA patient samples reveal a unique processing signature in a patient where RPL5 is mutated.

Total RNA from stimulated blood mononuclear cells was transferred to zeta probe membrane and hybridized with probes listed in Table 1. Names of probes used in the labeling of the Northern blots are shown at the top of each panel. The gene mutated in each sample is listed above each lane, with the healthy control samples listed as Wt. 
The 5S RNA subcomplex as a possible mediator of ribosome stress signaling

The decrease in 5S rRNA in samples from patient's haploinsufficient for $R P L 5$ has a number of implications for DBA pathophysiology. The 5S RNA is unique among the four rRNA species found in eukaryotic cytoplasmic ribosomes. It is the only rRNA transcribed independently by RNA polymerase III, as opposed to the other three rRNAs transcribed together by RNA polymerase I (Allison et al., 1995; Ciganda and Williams, 2011; Zhang et al., 2007). This unusual organization lends itself to the potential adaptation of 5S RNA and its associated proteins, RPL5 and RPL11, as a novel signaling subcomplex of the ribosome. In fact, many studies have suggested that ribosome stress is mediated through the interactions of RPL5 and RPL11 with MDM2, which results in stabilization and activation of p53 (Honda et al., 1997; Kubbutat et al., 1997). Additional work has implied that $5 \mathrm{~S}$ rRNA is involved in this process by forming a stable subcomplex with RPL5 and RPL11 and that it is the subcomplex that actually interacts with MDM2 in vivo as shown by the synergistic inhibition of MDM2 activity (Allison et al., 1995; Fumagalli et al., 2009; Horn and Vousden, 2008; Lipton and Ellis, 2010).

While the view that this ternary complex may be free to interact with MDM2 when large subunit assembly is disrupted by haploinsufficiency for any large subunit ribosomal protein other than RPL5 and RPL11 themselves, it is less clear why this complex may be freed to interact with MDM2 when small subunit assembly is affected. Therefore, we were interested in whether there may be an increase in free 5S RNA in samples where 40 S subunit assembly is disrupted by 
reduced expression of RPS19. To address this question, we turned to human TF-1 cells where RPS19 expression can be regulated by an inducible shRNA to RPS19 mRNA (Flygare et al., 2007). Fig. 9 shows polysome profiles from cells depleted of RPS19 and scrambled controls. Cells depleted of RPS19 show a decrease in free $40 S$ subunits, an increase in free 605 subunits, and a shift from larger to smaller polysomes. This is the expected outcome for a small subunit biogenesis defect. When $40 S$ subunits are limiting, both $40 S$ and $80 S$ species are decreased whereas free 60S subunits increase since $40 \mathrm{~S}$ pre-initiation complexes are no longer available. To monitor the fate of 5 S rRNA under these conditions, RNA was precipitated from the polysome fractions of these samples and then used for Northern blotting. Interestingly, the fraction of 5S rRNA in the free pool found at the top of the gradient is increased approximately 3-fold from $\sim 5$ to $15 \%$ in cells depleted of RPS19 relative to control samples. While not conclusive, these data suggest that increases in the 5S RNA subcomplex may occur in cells depleted of RPS19, and in doing so be available for signaling ribosome stress to regulators of $\mathrm{p} 53$. 


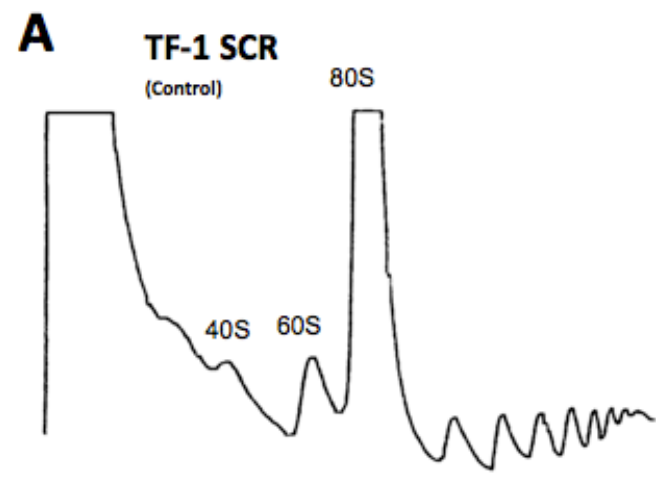

B

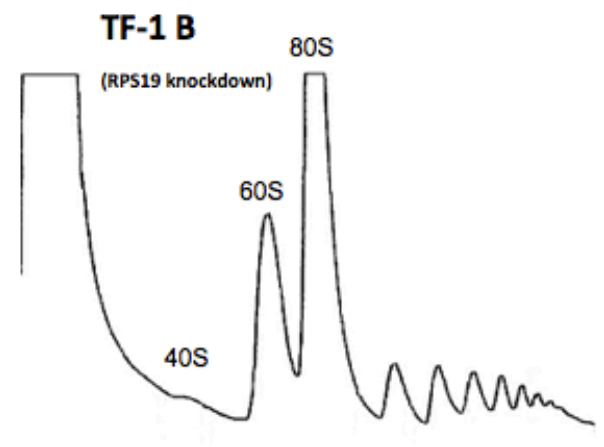

Free 5SrRNA 60S associated 5SrRNA

5S rRNA
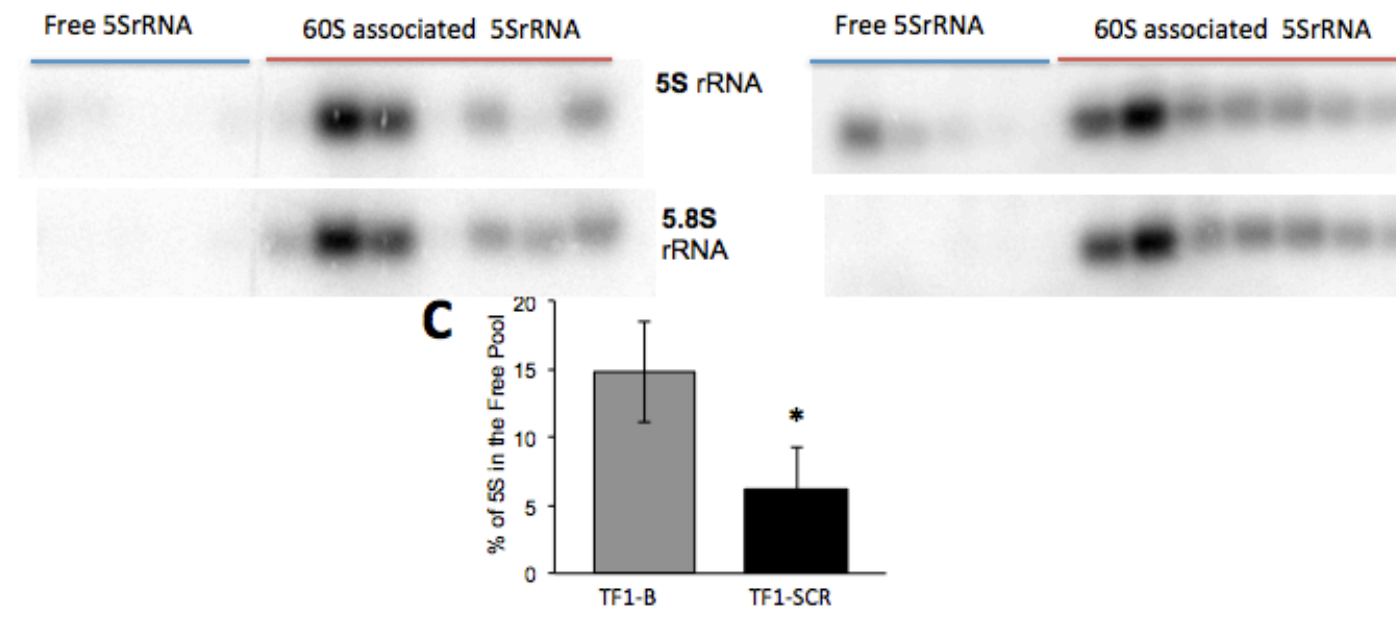

Figure 9: Polysome profiles of TF-1 samples depleted of RPS19 show an increase in the free pool of 5S rRNA.

TF-1 B and TF-1 SCR cells were treated with doxycycline for seven days to stimulate expression of shRNAs targeting RPS19 (TF1-B) shown in (B) or a scrambled control sequence (TF1-SCR) shown in (A). The resulting extracts were prepared in the presence of cycloheximide and separated by ultracentrifugation on sucrose gradients. Each panel shows representative absorbance tracings at $254 \mathrm{~nm}$ and Northern blots of gradient fractions probed with oligonucleotides complementary to either $5 \mathrm{~S}$ or $5.8 \mathrm{~S}$ rRNA are shown below. Panel C shows the fraction of $5 S$ rRNA found at the top of each gradient relative to the total amount of 5S rRNA across the gradients. Values for the amount of 
$5 S$ rRNA in each fraction were obtained by phosphorimage analysis using Imagequant (GE). Free 5S was determined by adding the band intensities of lanes delineated "Free 5S" above the Northern blots over the sum of the $5 S$ rRNA signals. Free 5.8S rRNA was quantified in a similar manner (data not shown), but with equivalent results in both control and knockdown samples. Error bars represent S.D. with $n=4$ and $a{ }^{*} p<0.05$ value as determined by an unpaired student's t-test. 
The amount of free 5S RNA in cells depleted of Rps19 was also investigated in a different model system of mouse embryonic stem cell lines (mES) where a single copy of the Rps19 gene is interrupted by insertion of a genetrap retroviral construct between exons 3 and 4 . This leads to a truncated mRNA transcript as outlined in the materials and methods chapter. The genetrapped embryonic stem cells were purchased from the Mutant Mouse Regional Resource Centers, MMRRC. The system was chosen by one of our collaborators to investigate the effects of heterozygosity for Rps19 on different aspects of mouse development in in vitro differentiation assays (see appendix). Extracts from the Rps19 gene trap and parental controls labeled YHC and E14, respectively were treated as described above for TF-1 cultures and analyzed in the same manner. The results showed the free pool of $5 \mathrm{~S}$ rRNA increased from $18 \%$ to $25 \%$ in the Rps19 gene trap samples relative to control (Fig. 10). Though not as striking a result as those found in the TF-1 model, ribosome defects in mouse models are often less severe then those in human cell lines (Flygare et al., 2007; Flygare et al., 2005; Gazda et al., 2004; Jaako et al., 2011). In contrast, mES models that recapitulate $R p / 5$ heterozygosity displayed essentially no change in the free pool of 5SrRNA (Fig. 11). 


\section{E14 (RPS19 Control)}

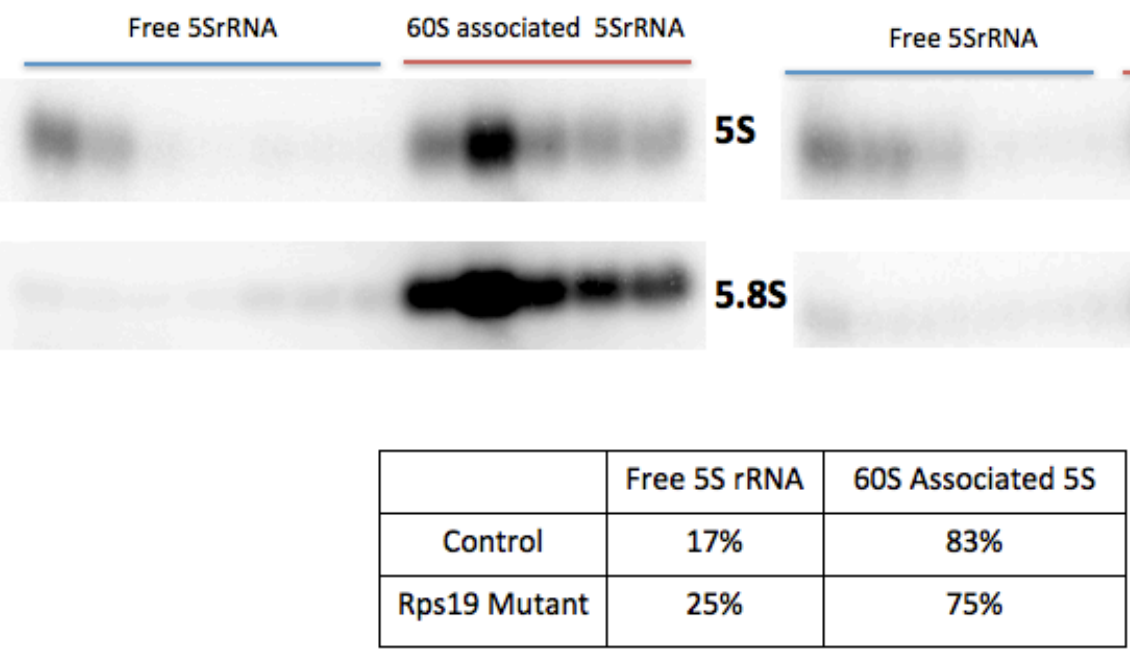

\section{YHC (RPS19 mutant)}
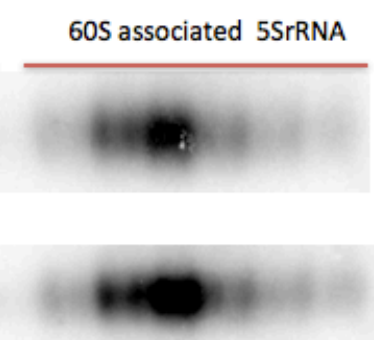

Fig 10: Free 5S rRNA is increased in mouse embryonic stem cells heterozygous for Rps19

RNA was precipitated from polysome fractions of YHC (Rps19 mutant) and their corresponding parental control (E14) mES lines. Northern blots of the RNA samples are shown in the top panels and the band intensity quantified in the lower panel's table using Imagequant (GE). Percentages of rRNA samples in each category were calculated by adding the band intensity of each of the lanes in the same category and dividing by the total band intensity. 


\section{YHC (Rpl5 control)}

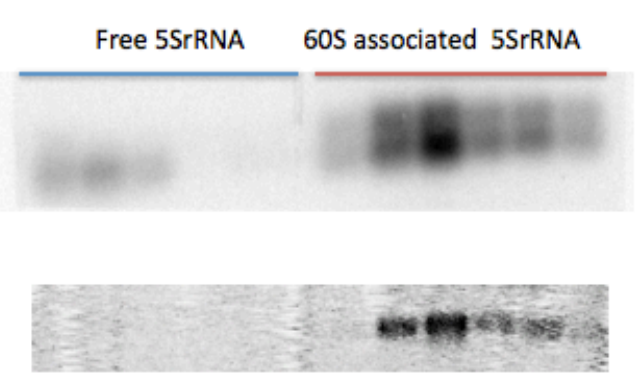

\section{D050 (Rpl5 mutant)}

5S

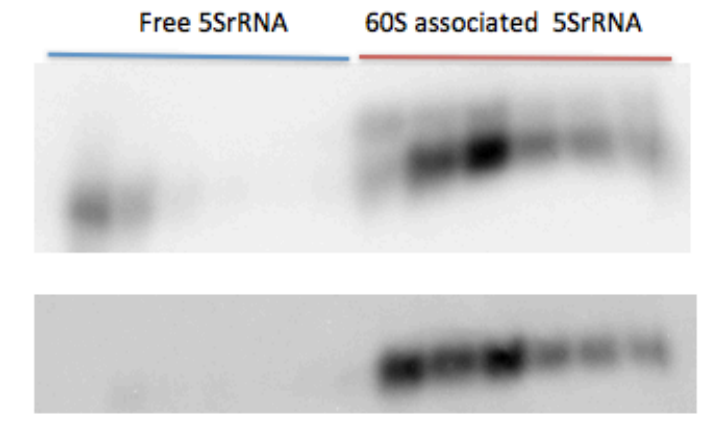

\begin{tabular}{|l|l|l|}
\hline & Free 5S & 60S Associated 5S \\
\hline Control & $15 \%$ & $85 \%$ \\
\hline Rpl5 Mutant & $16 \%$ & $84 \%$ \\
\hline
\end{tabular}

Fig 11: Free 5S rRNA is unchanged in mouse embryonic stem cells heterozygous for Rp/5

RNA was precipitated from polysome fractions of D050 (Rp/5 mutant) mES and their corresponding parental control $(\mathrm{YHC})$. Northern blots of the RNA samples are shown in the top panels with the quantification performed using Imagequant software (GE Healthcare) below. The amount of $5 S$ rRNA in the first six lanes were classified as the free pool, whereas that in lanes from 60S through the polysome fractions were considered 605 associated as localized by the cosedimintation of $5.8 \mathrm{~S}$ rRNA. The Table shows the relative amount of $5 \mathrm{~S}$ rRNA found in each fraction. 
The $5 S$ rRNA released with abortive assembly in our mES model of small subunit protein mutations is presumably free to interact with MDM2 (in association with Rpl5 and Rpl11) to prevent MDM2 mediated degradation of p53. In the case of the $R p / 5 \mathrm{mES}$ model, there is no corresponding increase in $5 \mathrm{~S}$ rRNA in the free pool. Nevertheless, there appears to be an increase in p53 protein expression in each of the gene trap cell lines (Fig. 12). In the S17 derived Rps19 mutant cell lines, this increase in p53 protein expression is evident in the undifferentiated mES cells (Fig. 12A). After stimulation to form embryoid bodies, as described in the supplemental section for use as a model of primitive hematopoiesis (Fig S4). The YHC Rps19 cell model, as well as the Rp/5 model, D050, shows an increase in p53 protein expression. These results are consistent with a model where p53 activation in cells haploinsufficient for Rpl5 occurs through a mechanism distinct from that of other ribosomal protein genes, excluding Rpl11. 

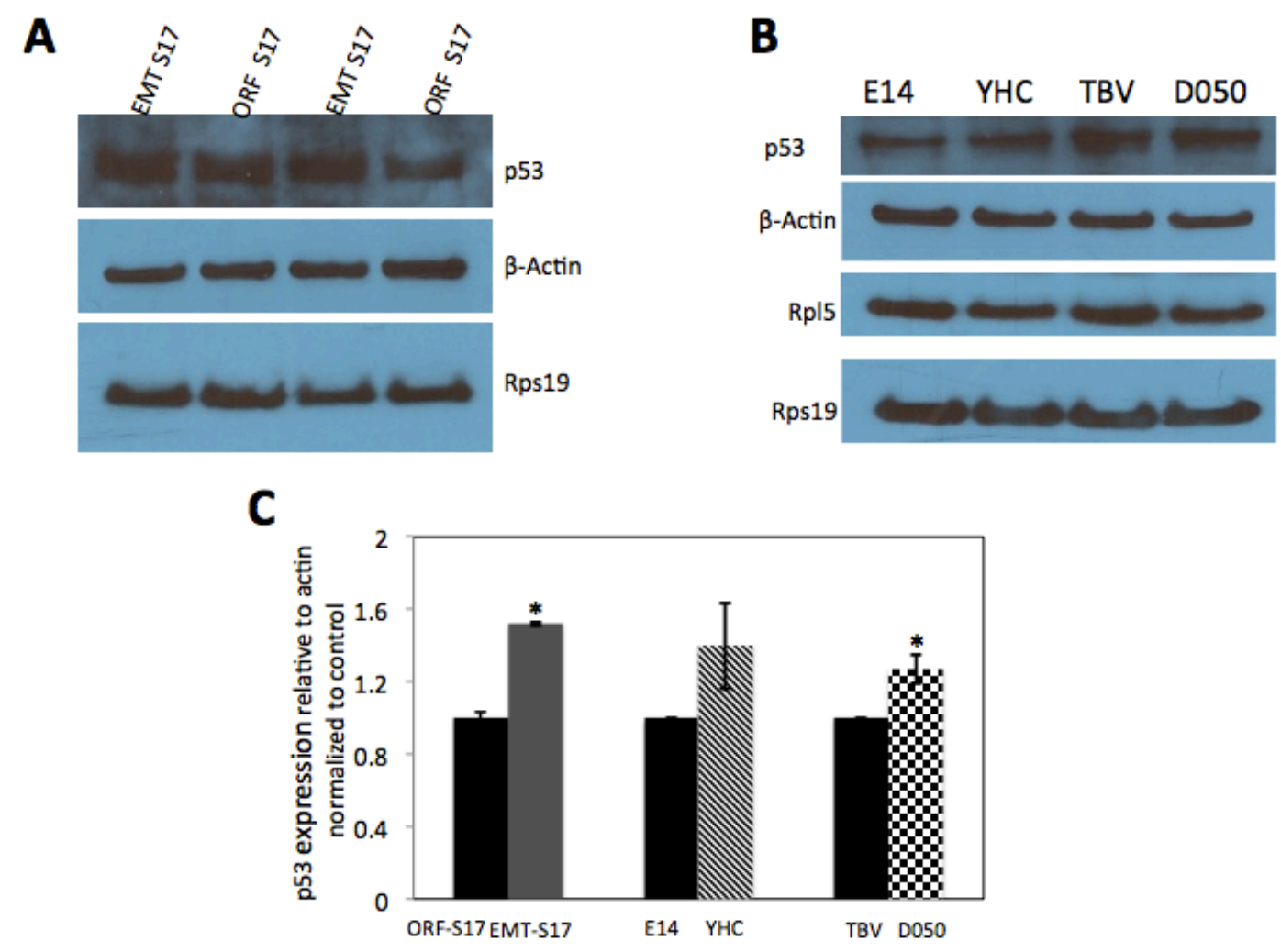

Fig 12: Cells heterozygous for either Rps19 or Rp/5 show an increase in p53 expression relative to controls.

(A) Duplicate samples of control (ORF-17) or Rps19 gene trap (EMT-S17) mES cells whole cell lysates were used for SDS-PAGE. Anti-sera for p53, $\beta$-Actin, and Rps19 were used for the western blots. (B) Rp/5 or Rps19 gene trap mES cells were differentiated into embryoid bodies and then harvested and whole cell lysates used for western blotting using anti-sera for $p 53, \beta$-Actin, and Rps19. (C) Band intensities of western blots were quantified using ImageJ. Data in the bar graph represent the means of multiple exposures of multiple experimental replicates, error bars $=S . D .,{ }^{*} p<0.05$ as determined by unpaired Student's $t$-test. 


\section{Discussion}

The case reported here illustrates some of the complexities associated with making a definitive diagnosis of an inherited bone marrow failure syndrome. The child presented with anemia and neutropenia with congenital anomalies characteristic of both Diamond Blackfan anemia and Fanconi anemia. The neutropenia could also suggest Shwachman Diamond syndrome (Shimamura and Alter, 2010). Fanconi anemia was ruled out, as the child's cells did not appear to be unusually sensitive to DNA crosslinking agents as would be expected for this disease (Shimamura and Alter, 2010). This sensitivity stems from the fact that the underlying molecular defect in FA resides in DNA repair at sites of double strand breaks (Soulier, 2011). Sequencing the SBDS gene and the 10 known pathogenic ribosomal protein genes associated with DBA in this child also revealed no obvious mutations, further complicating the potential of an accurate diagnosis.

The finding that this child had a 2.3 megabase deletion including the gene encoding ribosomal protein RPL31 suggested that she might indeed have DBA resulting from ribosomal protein haploinsufficiency. If confirmed, these studies would identify RPL31 as a new DBA gene. Demonstration of a ribosome biogenesis defect can help add to a DBA diagnosis, but analyses such as Northern blotting and polysome profiling are restricted to a few research laboratories and are unlikely to be adopted in clinical laboratories. This is, in part, due to the complicated equipment used to generate polysome profiles and the personnel hazards associated with the radiation used in common Northern 
blotting techniques. Having a facile assay that does not involve radioisotopes would greatly improve the prospects of monitoring for defects in ribosome synthesis as a diagnostic tool for DBA.

The data reported here clearly demonstrate that the proband in the index case has a pre-RNA processing pattern indicative of a defect in the maturation of the 605 subunit as would be expected for haploinsufficiency for a large subunit ribosomal protein. These results strongly support a diagnosis of DBA for this patient and also identify RPL31 as a new DBA gene (Campagnoli et al., 2008; Choesmel et al., 2007; Farrar et al., 2008; Gazda et al., 2008; Jaako et al., 2011; Robledo et al., 2008; Rouquette et al., 2005).

The studies reported here also show that patients with mutations in genes encoding large subunit ribosomal proteins have a common pre-rRNA processing defect visualized at the level of an ethidium bromide-stained gel. We strongly feel that this observation could be readily adapted to a routine assay for patients with defects in 60S subunit assembly, which could aide in DBA diagnosis and gene discovery efforts. This assay could be performed in a gel format as reported here, or could be readily adapted to testing in a Bioanalyzer, commonly used to test RNA integrity prior to quantitative PCR techniques.

An additional outcome of our investigation of pre-rRNA processing patterns in DBA patients with mutations in large subunit ribosomal protein genes was the demonstration that mature 5S rRNA is dramatically and selectively decreased in patients haploinsufficient for RPL5. RPL5 has been known for many years to bind directly to 5S rRNA (Allison et al., 1995; Thomas and Kutay, 
2003). Work in recent years has shown that RPL11 and two additional ribosome associated proteins are required for the incorporation of the $5 \mathrm{~S}$ subcomplex into assembling 60S subunits (Ciganda and Williams, 2011; Dai et al.; Grandi et al., 2002; Hadjiolova et al., 1993; Horn and Vousden, 2008; Kubbutat et al., 1997; Lohrum et al., 2000; Lohrum et al., 2003; Zhang et al., 2007).

In addition to its role as a vital component of the 605 subunit, increasing data suggest that this subcomplex also plays a critical role in ribosomal stress signaling. The complex of 5S rRNA together with RPL5 and RPL11 has been shown to bind to the ubiquitin ligase MDM2 and inhibit its activity toward p53 resulting in an increase in the amount and activity of p53 (Dai et al., 2007a; Dai and Lu, 2004; Dai et al., 2007b; Dai et al., 2006; Dai et al.; Dai et al., 2004; Montanaro et al., 2007; Sun et al., 2007). This ribosome stress signaling pathway has been reported in several studies to play an important role in the proapoptotic phenotype of erythroid precursors in DBA (Badhai et al., 2009; Dai and Lu, 2008; Danilova et al., 2008; Horos et al., 2012; Jaako et al., 2011; Kuramitsu et al., 2008; Miyake et al., 2008; Ofir-Rosenfeld et al., 2008; Uechi et al., 2008).

Consistent with this view, our data indicate that the level of free 5S rRNA (presumably in complex with RPL5 and RPL11) increases even in cells where small subunit ribosomal proteins are depleted in both a human cell line and in mouse embryonic stem cell models of DBA. Thus, the $5 \mathrm{~S}$ rRNA subcomplex may play a critical role in ribosome stress signaling and thereby an important role in the pathophysiology of most cases of DBA. 
A critical role of the $5 \mathrm{~S}$ rRNA subcomplex in ribosome stress signaling in DBA patients seems at odds with the finding that both RPL5 and RPL11 are DBA genes. Our observation that $5 S$ rRNA is dramatically decreased in patient samples from a patient haploinsufficient for RPL5 strongly suggests that the $5 S$ rRNA subcomplex would be limiting for 605 subunit formation leaving little available for stress signaling. This apparent paradox could be reconciled if there were an alternative pathway used to signal ribosome stress in such cells. Such a pathway could dominate in cells where the 5 S rRNA subcomplex was unavailable such as in patient's haploinsufficient for RPL5 or RPL11. Interestingly, this alternative pathway may have characteristics distinct from the 5S rRNA subcomplex in ribosome stress signaling as DBA patients with mutations in RPL5 and RPL11 tend to have more severe forms of the disease with a greater spectrum of congenital anomalies including cleft lips and palates (Gazda et al., 2008). The existence of this alternative pathway in patients with RPL5 mutations is supported by the observation that p53 levels increase in the cells without an increase in the pool of free 5S rRNA (Fig. 11-12).

As the patient reported here is the first to have a mutation in $R P L 31$ it is too soon to tell if all of her clinical characteristics stem from the loss of RPL31. It is possible that some of her clinical features may be caused by loss of other genes in the deleted region. As RPL31 will likely now be included for sequence analysis in DBA patients lacking mutations in other known genes, it is possible that other patients will be identified that only have mutations in this gene. The clinical features of such patients will help resolve this issue. 


\section{Conclusions and Future Directions}

This work identifies RPL31 as a new potential DBA gene and adds to the growing list of ribosomal protein genes known to be mutated in this puzzling bone marrow failure. These studies represent the first description of a possible diagnostic assay for DBA patients with mutations in large subunit ribosomal protein genes based on the visualization of increased 32S pre-rRNA with UV light in standard RNA gels. The straight-forward nature of this assay could lend itself to high throughput methods more amenable to a clinical diagnostic laboratory such as bioanalyzers routinely used for assaying RNA quality for reverse transcription PCR.

After identification of this patient as a non-classical DBA patient, the child and her family, can obtain information, support, and guidance associated with the already existing infrastructure of the North American DBA Registry. Though the patient whose samples containing a deletion of RPL31 made this research possible died during the course of this study, her family will remain a part of the DBA family. May this research be a celebration of her life! 


\title{
CHAPTER IV
}

\section{MITOCHONDRIAL DYSFUNCTION IN A HUMAN CELL LINE MODEL OF SHWACHMAN DIAMOND SYNDROME}

\author{
$\underline{\text { Introduction }}$
}

Shwachman Diamond syndrome (SDS; OMIM 260400) is one of a class of inherited bone marrow failure syndromes (IBMFS). Its clinical presentation typically includes exocrine pancreatic insufficiency, cartilage disorders, and neutropenia, though deficits in multiple blood lineages have been seen in patients (Burwick et al., 2011). Patients with SDS have a greatly increased risk for myelodisplastic syndromes (MDS) and acute myelogenous leukemia (AML). Some studies estimate up to 40 percent of SDS patients will be affected MDS/ AML by age 30 (Donadieu et al., 2005; Dror and Freedman, 2002; Shimamura and Alter, 2010; Smith et al., 1996; Tamary et al., 2010). Over $90 \%$ of SDS patients have pathogenic mutations in the gene SBDS (Shwachman Bodian Diamond Syndrome) (Boocock et al., 2003). The majority of mutations are located in exon two with many resulting from gene conversion with the adjacent pseudogene (Burroughs et al., 2009). The diverse biological systems affected by mutations in SBDS suggest that SBDS may be a multifunctional protein. SBDS is reported to have many functions including ribosome biogenesis (Austin et al., 
2005; Finch et al., 2011; Ganapathi et al., 2007; Menne et al., 2007; Moore et al., 2010; Orelio et al., 2011; Vitiello et al., 2010; Wessels et al., 2006; Wong et al., 2011), maintenance of bone marrow stromal environment (Leung et al., 2011), chemotaxis, mitotic spindle stability (Austin et al., 2008; Orelio and Kuijpers, 2009; Orelio et al., 2009) and regulating apoptosis pathways (Ambekar et al., 2010; Rujkijyanont et al., 2008; Watanabe et al., 2009).

Much of our current knowledge regarding SBDS function in ribosome biogenesis was obtained using yeast models lacking SDO1, the yeast ortholog of $S B D S$. One possible explanation for the multiple functions associated with the loss of SBDS in human cells is that some of these properties are a consequence of downstream effects of the loss of members of the SBDS protein family on translation output. To address this issue, Dr. Joseph B Moore IV in our laboratory analyzed the effect of loss of SDO1 on translation in yeast. To this end, cells were pulse labeled for 30 minutes with [ $\left.{ }^{35} \mathrm{~S}\right]-$ Met and proteins resolved by twodimensional gel electrophoresis. Fig. 13 shows the results of this analysis. One of the proteins shown to be substantially increased in $\triangle S D O 1$ cells relative to controls was identified as Por1, the yeast ortholog of VDAC1, a voltage dependent anion channel of the mitochondrial outer membrane (McDonald et al., 2009). This observation led us to the finding that yeast cells lacking Sdo1 were incapable of growing on non-fermentable carbon sources indicating that they had defective mitochondrial function. The studies described in this chapter are focused on mitochondrial function in a human erythroleukemia cell line depleted of SBDS. The first portion of this chapter will discuss the development and 
characteristics of a human cell line depleted of the SBDS protein, while the remaining studies will focus on testing the hypothesis that mitochondrial function may be disrupted in cells depleted of SBDS. 


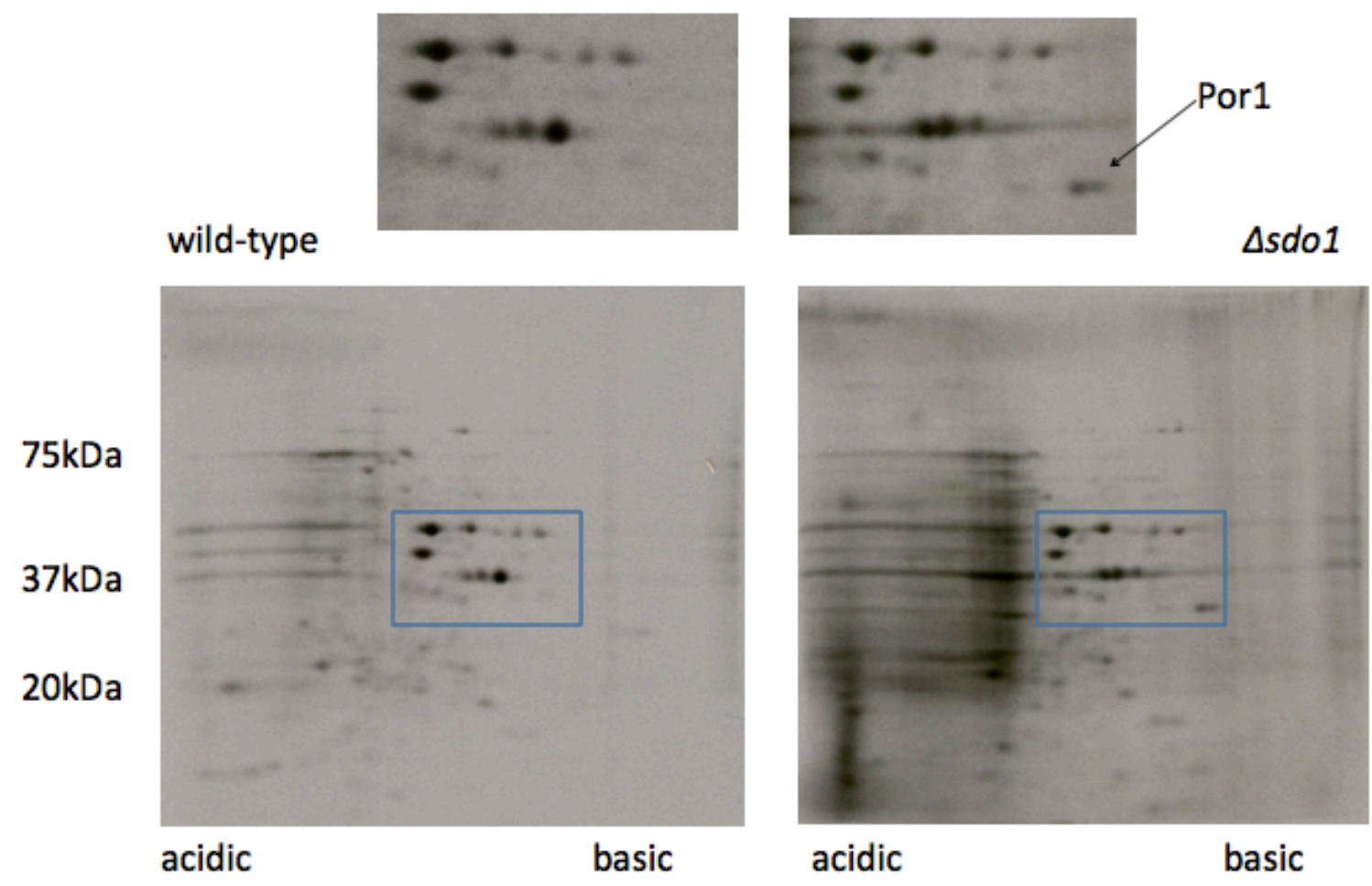

Fig 13: Por1 expression is increased in $\triangle$ SDO1 yeast strain

Wild-type (left panels) and $\Delta$ SDO1 (right panels) strains were incubated for 30 minutes with $\left[{ }^{35} \mathrm{~S}\right]-$ Methionine. Total protein extracts were resolved by $2 \mathrm{D}$ gel electrophoresis. The $\mathrm{pH}$ gradient for IEF was 3-10. The second dimension was resolved via a $4-12 \%$ polyacrylamide gel. Molecular masses for size standards are shown to the left. Regions enclosed by the boxes are expanded above each gel. The spot labeled Por1 was excised and identified via MALDI-TOF mass spectrometry. 


\section{$\underline{\text { Results }}$}

Targeting SBDS gene expression in the human erythroleukemia TF-1 cell line

Our goal was to create a tissue culture model for Shwachman Diamond syndrome by using shRNA technology to reduce expression of SBDS in a human cell line. This approach was necessary given the limited availability of patient samples for this rare bone marrow failure syndrome. SBDS is a multifactorial bone marrow failure that has been shown to affect numerous blood cell lineages, so the TF-1 erythroleukemic cell line was chosen for its ability to differentiate along various myeloid lineages (Dror and Freedman, 1999; Leung et al., 2011; Miyake et al., 2005; Rawls et al., 2007; Sen et al., 2011; Wong et al., 2011).

Steady-state levels of SBDS were monitored in cell lines transduced with lentiviral vectors expressing shRNAs to SBDS or scrambled controls. To this end, whole cell extracts were prepared, proteins fractioned by SDS-PAGE, transferred to nitrocellulose filters and immunoblotted with anti-sera raised against SBDS, or $\beta$-actin as a loading control. Fig. 14A shows that relative to scrambled controls the steady-state amount of SBDS was reduced to near undetectable levels. Reduced SBDS expression was also observed at the mRNA level as determined by qRT-PCR (Fig. 14B). SDS is an autosomal recessive disease with most patients expressing very low levels of SBDS, typically from hypomorphic alleles. Consequently, only clones expressing SBDS at levels below $30 \%$ of wild-type were used for subsequent studies. 


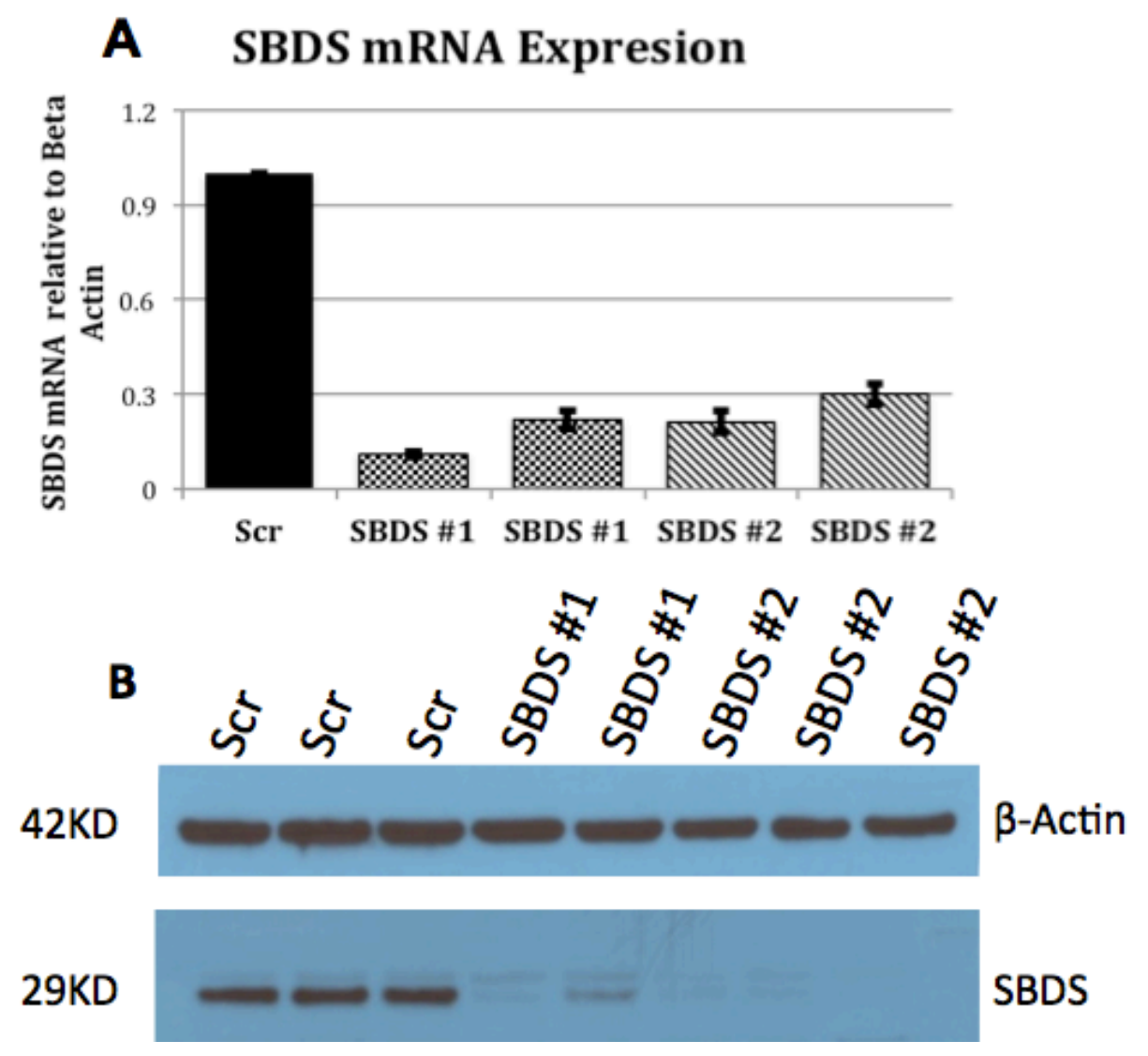

Fig 14: Steady-state amounts of SBDS protein and mRNA in TF-1 clones. (B) Immunoblot of whole cell lysates of TF-1 cells transduced with lentiviruses expressing shRNAs against SBDS mRNA or scrambled controls. Membranes were blotted with anti-sera raised to SBDS or $\beta$-actin. (A) qRT-PCR of SBDS mRNA from total RNA isolated from lentiviral transduced TF-1 cells. Each bar in the graph represents a different clone created during the selection process with the numbers corresponding to the sequence of the SBDS plasmid used to transduce the TF-1 cells. 
Reduced SBDS expression slows growth rate and affects 605 subunit maturation

TF-1 cells depleted of SBDS protein have a slower growth rate when compared to the scrambled control samples (Fig 15) in congruence with previous cell models of SDS (Menne et al., 2007; Moore et al., 2010). The reduced growth rates of human cell line models of SDS have been correlated with adverse effects on the maturation of 605 ribosomal subunits and translation. This should come as no surprise with loss of a protein that is integral in 605 subunit maturation. The status of the translational machinery in our model system was investigated by a technique called polysome profiling. Extracts suitable for polysome profiling were prepared by harvesting cells in the presence of cycloheximide, which inhibits the elongation phase of translation and traps ribosomes on mRNAs. Extracts prepared in the presence of cycloheximide were then subjected to sucrose gradient centrifugation. Gradients were fractionated and absorbance at $254 \mathrm{~nm}$ was monitored. A representative absorbance tracing is shown in (Fig 16) where cells depleted of SBDS exhibit a modest decrease in free 605 subunits and $80 S$ subunits. More importantly, the decreased absorbance in the polysome region indicates a defect in the formation of mature ribosomes that are actively participating in translation. 


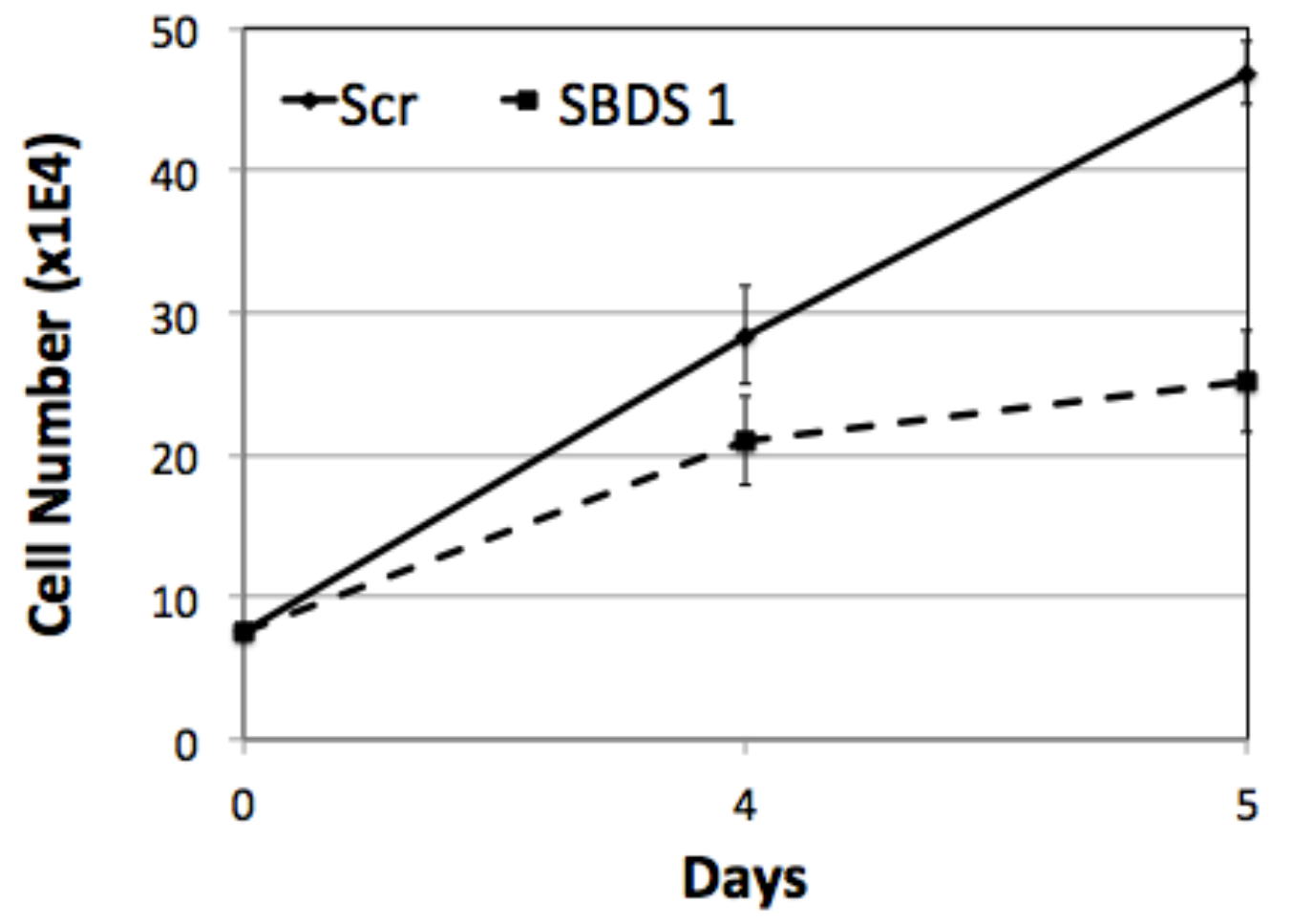

Fig 15: Cells depleted of SBDS have a reduced growth rate relative to scrambled controls.

TF-1 cells treated with lentiviral vectors targeting SBDS expression (dashed line) or a scrambled control (solid line), were counted using trypan blue exclusion for cell viability. ( $n=3$, error bars represent S.D.). 


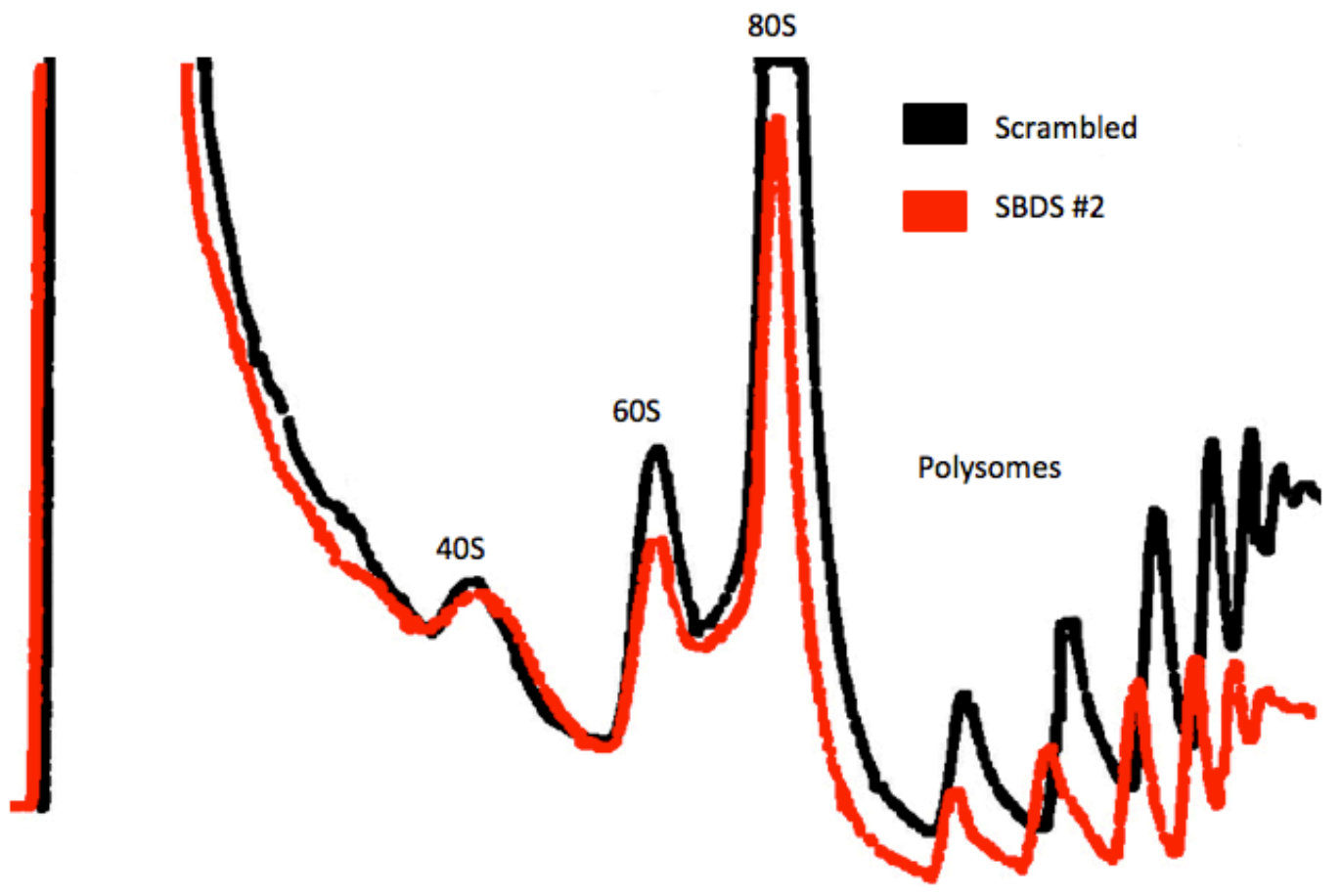

Fig. 16: Polysome profiles from TF-1 cells depleted of SBDS and a scrambled control.

The above image is a representative absorbance tracing at $254 \mathrm{~nm}$ of TF- 1 cells fractionated by sucrose gradient centrifugation. Black: scrambled control; Red: shRNA to SBDS \#2. Cells were harvested in the presence of cycloheximide as previously described (16). The absorbance tracing was captured using an ISCOUV/Vis detector UA-6 and ISCO optical unit type II. Samples were normalized relative to the trough following the $40 \mathrm{~S}$ subunit peak. This result was recapitulated in four independent experiments. 
Previous results have demonstrated a role for SBDS in the release of elF6 from 605 subunits. The elF6 protein associates with pre-60S subunits in the nucleolus and plays an important role in transporting pre-60S particles from the nucleus to the cytoplasm. The elF6 also has anti-subunit association activity which prevents 605 subunits from joining with 40 S subunits before they are fully matured. The failure to release elF6 from 60 S subunits in the absence of SBDS prevents 605 subunits from prematurely binding to $40 S$ subunit during formation of the 80 S initiation complex (McDonald et al., 2009; Nihrane et al., 2009; Rujkijyanont et al., 2008). To monitor the relative distribution of elF-6 between free and 60S subunit-bound pools, gradient fractions were immunoblotted for the presence of elF6. Antibodies against RPS19 and RPL5 were used as controls to identify peaks for the small and large subunit, respectively (Fig. 17, top panel). These data revealed an approximately $20 \%$ increase in elF6 co-sedimenting with $60 S$ subunits in cells depleted of SBDS. This increase in association with the 60S subunit was shown to be reproducible and statistically significant $(n=4, p=0.001$ as determined by an unpaired Student's t-test). 


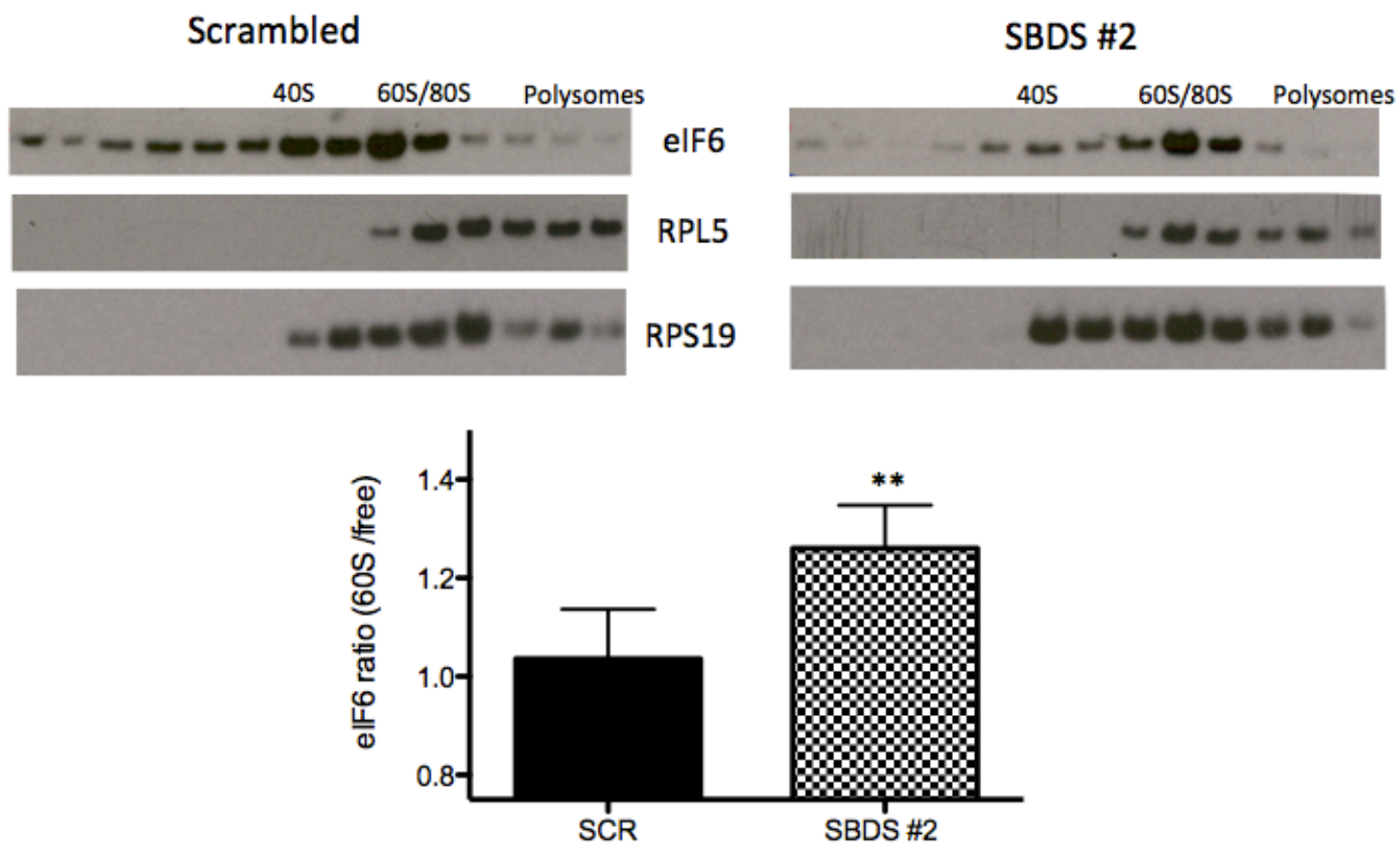

Fig. 17: Distribution of elF6 in sucrose gradients derived from TF-1 cells depleted of SBDS and scrambled controls.

Top panel: representative immunoblots of proteins precipitated from sucrose gradient fractions of TF-1 cells transduced with the Sigma Aldrich SBDS shRNA \#2 or scrambled (Scr) lentiviral constructs. The fractions on the far left correspond to the top of a gradient. Fractions were precipitated with $10 \%$ trichloroacetic acid, washed, resuspended in SDS sample buffer, and 1/10 of each fraction was loaded on a gel for subsequent immunoblotting. Anti-sera to ribosomal proteins RPS19 and RPL5 were used to identify fractions containing $40 S$ and 605 subunits, respectively. $60 \mathrm{~S}$ subunits and $80 \mathrm{~S}$ monosomes were not completely resolved in this experiment. The signal intensity in immunoblots were quantified using ImageJ software. Data in the bottom panel are plotted as a 
ratio of elF6 co-sedimenting with 60 S subunits over elF6 present in remaining fractions at the top of the gradient. The percentage of elF6 co-sedimenting with the 60 subunit fraction was increased by about $20 \%$ relative to controls in cells depleted of SBDS (1.26 in the SBDS \#2 samples and 1.04 in the control samples, $n=4$, column $=$ mean, error bars $=S E M,{ }^{* *} p \leq 0.01$ as determined by an unpaired Student's t-test). 


\section{$\underline{\text { SDS model exhibits cell cycle delay and maturation defects }}$}

Previous work with cellular models of SBDS has shown a delay in cell cycle progression (Ambekar et al., 2010; Ball et al., 2009; Wong et al., 2011). To further validate our model, cell cycle progression was analyzed by flow cytometry after permeabilizing cells and treating with RNAse and propidium iodide. A small but statistically significant delay in cell cycle progression was observed in cells depleted of SBDS. This delay is observed as an average increase of cells in G0/ G1 of approximately $6 \%$, with a corresponding decrease in the percentage of cells in S phase (Fig. 18). 

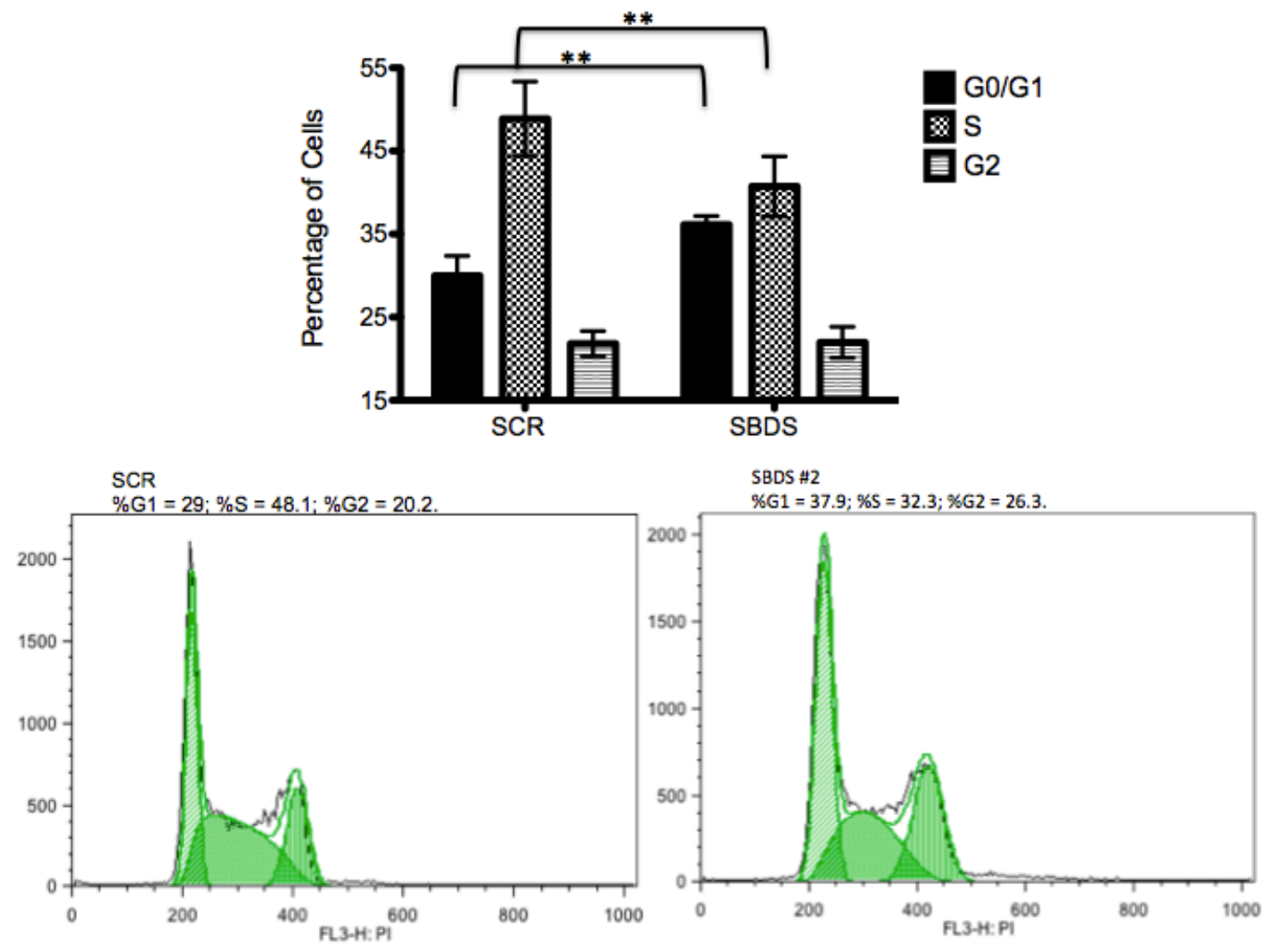

Figure 18: Analysis of cell cycle progression in cells depleted of SBDS and scrambled controls.

Cells depleted of SBDS and scrambled controls were stained with propidium iodide and cell cycle phase was monitored by flow cytometry in the FL3H-PI channel.

Top panel: The average of six independent experiments with the standard deviation used for error bars. Data were compared by ANOVA, the difference between cells present in G0/G1 relative to $S$ was calculated using an appropriate post-hoc test as $8 \%$, with ${ }^{* *} p<0.01$. 
Bottom panel: representative histograms with the shaded regions depicting the most likely mathematical model as determined by the Dean/Jett/Fox method using FlowJo software. The first peak, containing 2N DNA content represents cells in the G0/G1 phase. The peak at the far right represents cells that have $4 \mathrm{~N}$ DNA content and are in the G2/M phase of the cell cycle. The intermediate cells are in the synthesis phase and have varying DNA content. The black outline shows the histogram of raw data generated with the mathematical algorithm of the Dean/Jett/Fox method calculating the most likely peak representations of the three cell populations. 
The depletion of SBDS in TF-1 cells also gave us an opportunity to determine the effect of this depletion on the ability of these cells to differentiate along the erythroid and granulocyte lineages. TF-1 cells can be induced to differentiate along the myeloid or erythroid lineages in response to the cytokines GM-CSF (granulocyte and macrophage colony stimulating factor) and EPO (erythropoietin), respectively. Differentiation is evaluated by colony formation in methylcellulose media. A cluster is considered a "colony" if it contains 40 or more cells and plating density is such that that many cells in a cluster have originated from a single progenitor (Miyake et al., 2005). This is analogous to the bone marrow derived hematopoietic stem cells giving rise to committed progenitors that form colony forming units (CFUs) and as they commit to a particular lineage, in this case burst forming units of the erythroid, or granulocyte lineage, BFU-E and GM-CFU respectively. These cells then continue to grow as clumps derived from the same progenitor that divide to form a clone or further differentiate (Metcalf, 2007). The viscous medium provides a matrix for the mutli-potent cells to differentiate toward a particular cell lineage based on which cytokines are used to stimulate them. This is a common experimental model used to investigate IBMFS that have a delay or block in maturation of specific blood lineages. Fig. 19 shows a decrease in the ability of SBDS-depleted TF-1 cells to differentiate down both erythroid and granulocyte lineages consistent with defects seen in SDS patient samples performed by Dr. Sharon Singh in the laboratory of Dr. Johnson Liu (Nihrane et al., 2009; Orelio et al., 2009; Rawls et al., 2007; Sen et al., 2011; Yamaguchi et al., 2007). 


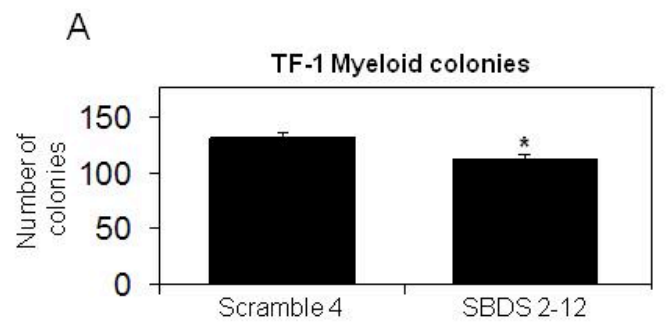

C

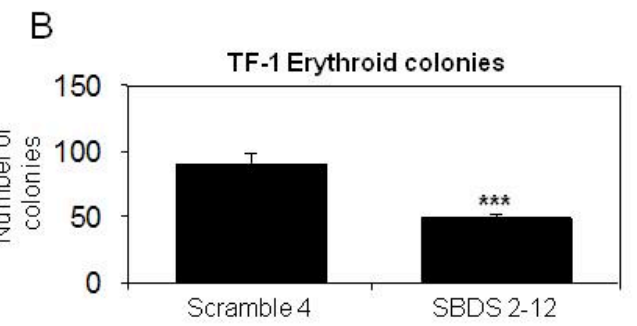

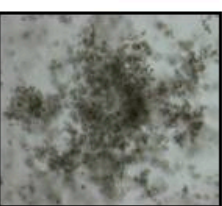

Scramble 4

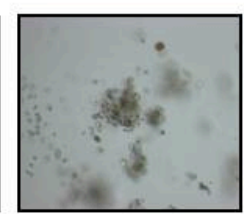

SBDS 2-12

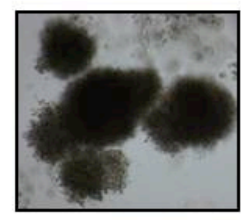

Scramble 4

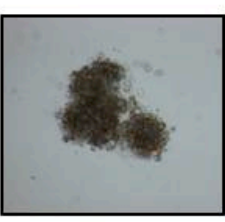

SBDS 2-12

Figure 19: Analysis of erythroid and granulocyte differentiation of TF-1 cells depleted of SBDS or scrambled controls

TF- 1 cells were differentiated into either myeloid or erythroid colonies using GMCSF or EPO in methylcellulose media. The \#2-12 SBDS knockdown clone had decreased myeloid (A) and erythroid (B) colony formation when compared with the scrambled \#4 control. Markedly smaller colonies were also seen in the \#2-12 clone (C) and (D) . ${ }^{*} p=0.0040{ }^{* * *} p=0.0004$. 


\section{Changes in oxygen consumption in SDS model}

Previous results in our laboratory showing that yeast $\Delta s d o 1$ strains were unable to grow on non-fermentable carbon sources sparked our desire to address aspects of mitochondrial function in TF-1 cells depleted of SBDS. In yeast, it was possible to assess mitochondrial function by monitoring growth on non-fermentable carbon sources. Similar experiments cannot be performed in human cells. Consequently, we used a number of alternative strategies to assess mitochondrial function in TF-1 cells depleted of SBDS.

One of these strategies was to monitor oxygen consumption. Oxygen consumption was monitored using a Gilson type oxygraph with a Clark-type electrode (Campian et al., 2007; Gao et al., 2010). These experiments were performed with the equipment and guidance of Dr. John Eaton's laboratory. Fig. 19 shows that cells depleted of SBDS consumed less oxygen compared to scrambled controls. Both basal and maximal rates of oxygen consumption are decreased, as evidenced by a difference in slopes between the two tracings in Fig. 20A both before and after the addition of the uncoupling agent CCCP. A greater effect on basal respiration is evident when the ratios of basal to maximal respiration are taken into consideration in the graphs depicting the averages of three independent experiments (Fig 20B). 


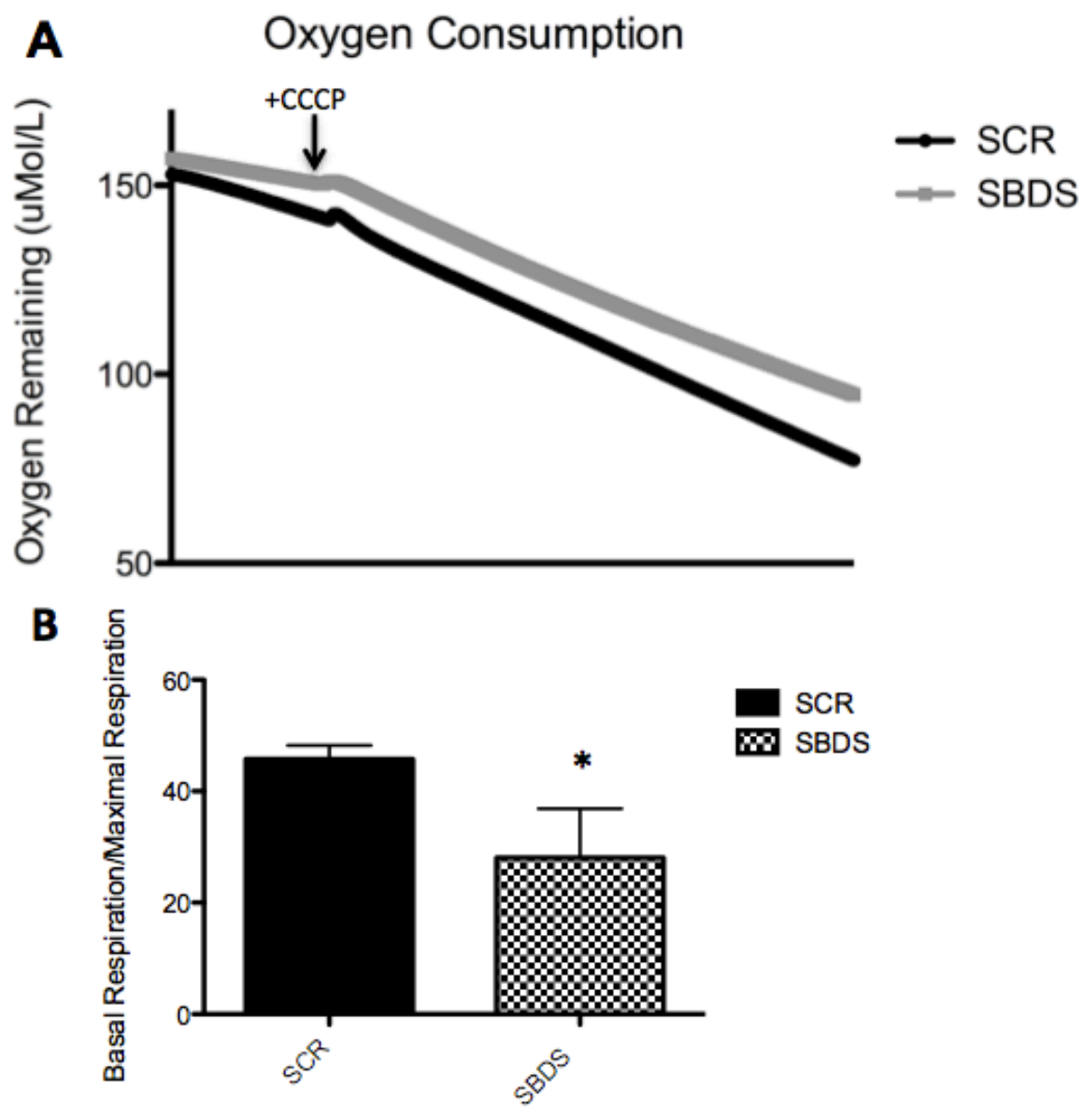

Fig 20: Oxygen consumption by TF-1 cells depleted of SBDS compared with scrambled controls.

A. Representative oxygen concentration tracings with time before and after addition of $10 \mathrm{mM}$ CCCP, B. Data from panel A were converted to $\mu \mathrm{mol}$ of oxygen consumed per million cells per minute and plotted as ratio of basal over maximum oxygen consumption rate. Averages of three independent experiments are shown with error bars showing S.E.M. $\left({ }^{*} p<0.05\right.$ as determined by an unpaired Student's t-test) 


\section{Changes in mitochondrial membrane potential and mitochondrial protein}

\section{expression with SBDS knockdown}

Oxygen consumption is linked to ATP synthesis in mitochondria through the chemiosmotic membrane potential created by protons pumped from the mitochondrial matrix to the inner membrane space via electron transport. Given the decrease in oxygen consumption in TF-1 cells depleted of SBDS, we were interested in whether this finding could be linked to a decrease in mitochondrial membrane potential (Wurm et al., 2011). The Mitoprobe JC-1 assay was used to examine mitochondrial membrane potential in TF-1 cells depleted of SBDS and scrambled controls. Cells treated with the JC-1 dye were analyzed by flow cytometry. The JC-1 dye undergoes a membrane potential dependent shift in emission spectrum from green $(529 \mathrm{~nm})$ to red $(590 \mathrm{~nm})$ as it enters and accumulates within the mitochondrial matrix (Chaoui et al., 2006; Chazotte, 2011; Cossarizza and Salvioli, 2001; Garner and Thomas, 1999; Guthrie and Welch, 2008; Keil et al., 2011; Legrand et al., 2001; Lugli et al., 2007; Reers et al., 1995; Salvioli et al., 1997; Simeonova et al., 2004; Smiley et al., 1991). Mitochondria with normal membrane potential should be able to retain a significant quantity of the dye and stain intensely in the red emission spectrum and fluorescence in the green emission spectrum validates the use of appropriate concentration of the compound were used in staining.

After gating to remove debris and dead cells in a separate plot of side scatter (SSC) and forward scatter (FSC) such that these signals were not included in the analysis a minimum of 40,000 events were counted in each 
experimental replicate. Data were plotted with red fluorescence on the $\mathrm{Y}$ axis (captured with the FL2H channel) and green on the $\mathrm{X}$ axis (captured with the FL1H channel). Quadrants were centered such that greater than $97 \%$ of the unstained control sample was found in the lower left quadrant. Positive controls were created in each replicate by pre-treating cells with CCCP, an electron transport chain uncoupler which should cause the mitochondrial membrane potential to greatly decrease. These samples were found predominately in the lower right quadrant. Thus, the majority of cells will be located in one of two quadrants. Cells highly fluorescing in both the green and red spectrum, as would be expected of cells with normal mitochondrial membrane potential, are found in the upper right quadrant and cells with lower membrane potential will be in the lower right quadrant because they have more intense fluorescence emissions in the green rather than the red emission spectrums corresponding to cells with a proportionally higher amount of $\mathrm{JC}-1$ dye in the cytoplasm rather than retained inside the mitochondria.

The bulk of cells in the control population treated with JC-1 are found in the upper right quadrant with relatively high red and green staining and so represents normal mitochondrial membrane potential for TF-1 cells (Fig. 21A). Cells depleted of SBDS show a significantly different staining pattern with a significantly decreased level of red fluorescence in the majority of cells (Fig. 21B). This shift is consistent with a decrease in mitochondrial membrane potential in cells depleted of SBDS. Three independent experiments were analyzed to determine relative fractions of normal mitochondria (based on their 
location in the upper right quadrant) in cell depleted of SBDS relative to controls. The results from these analyses are shown in Fig. $21 \mathrm{C}$. On average $72.8+/-3 \%$ of scrambled samples were found in the upper right quadrant representing TF-1 cells classified as having normal mitochondrial membrane potential. In contrast, $57.5 \%+/-2 \%$ of SBDS knockdown cells were found in this quadrant with a corresponding shift to the lower right quadrant representing cells with decreased mitochondrial membrane potential (Fig. $21, n=12-14$ and range $=$ S.E.M., $p$ value $<0.001$ as determined by Student's two-tailed t-test). A Fisher's exact test to assess whether the use of Student's t-test was appropriate for these analyses found the variance between the two means to lack significance, making the Student's t-test an appropriate choice for statistical analysis. When individual clones were compared using one-way repeated measures ANOVA, the means between different clones were also found to be statistically significant and a Dunnett's multiple comparison post-hoc test with $p$ values all less than 0.01 . 

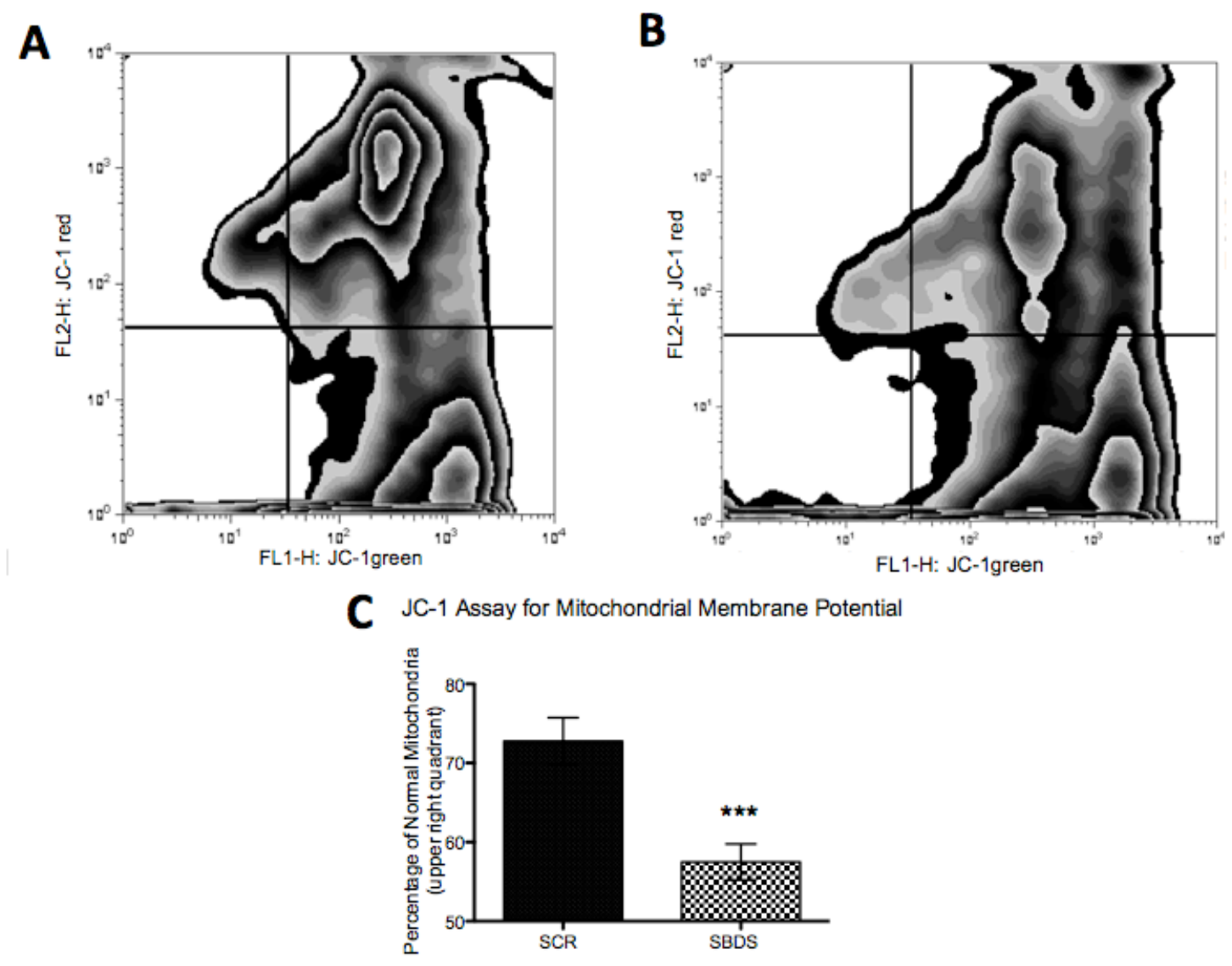

Fig 21: Analysis of mitochondrial membrane potential in cells depleted of

\section{SBDS and scrambled controls}

Flow cytometric analysis of cells treated with $\mathrm{JC}-1$, a cationic dye that accumulates in mitochondria in a membrane potential-dependent manner. The JC-1 dye undergoes a fluorescence emission shift from green $(529 \mathrm{~nm})$ to red (590 nm) upon entry into mitochondria. Representative histograms are shown where FL2-H: JC-1 red fluorescence is plotted on the y-axis and FL1-H: JC-1 green fluorescence on the x-axis. (A) scrambled control TF-1 cells treated with JC-1 (B) TF-1 cells depleted of SBDS treated with JC-1. Samples were analyzed using FlowJo software as outlined in the Materials and Methods section. Data are plotted as a contour map with cell densities denoted by color and peaks 
evident by decreased separation between contour lines. (C) Replicate data from three independent experiments are plotted as the percentage of TF-1 cells containing normal mitochondria as delineated by the upper right quadrant. Error bars represent S.E.M. $\left(n=3,{ }^{* * *} p<0.001\right.$ as determined by an unpaired Student's t-test) 
The data presented thus far indicate that cells depleted of SBDS have reduced oxygen consumption and reduced mitochondrial membrane potential. This decrease in mitochondrial function could arise from decreased mitochondrial mass within the cell or be the consequence organelles with reduced function. To assess levels of total mitochondria between cells depleted of SBDS and scrambled controls, we examined the expression of a number of mitochondrial proteins found in various locations within the organelle (see Table 2 for proteins examined). Unfortunately, no consistent patterns of expression were found in this analysis. Tom 20 and Tom40 represent components of the mitochondrial import machinery and might be classified as more or less constitutive components of the organelle. Despite being part of the same complex, the amount of Tom40 increased in cells depleted of SBDS relative to controls whereas the level of Tom20 went down (Fig. 22). Similarly, SOD2 and IDH2, two enzymes involved in reducing oxidative stress, also revealed discordant responses with SOD2 showing decreased expression with IDH2 increasing (see Fig. 21 for representative data). Interestingly, two enzymes involved in energy metabolism, pyruvate dehydrogenase (PDH) and isocitrate dehydrogenase, both decreased in expression. The same is true for cytochrome $\mathrm{c}$ which plays an important role in energy metabolism as a component of the electron transport chain. Finally, the expression of VDAC1 went up in cells depleted of SBDS consistent with the results in yeast, where Por1, the yeast ortholog of VDAC1, was increased in cells depleted of Sdo1 (Fig 23). 


\begin{tabular}{|c|l|c|c|}
\hline Protein & Location & Function & $\begin{array}{c}\text { Expression } \\
\text { change }\end{array}$ \\
\hline Tom20 & OMM & $\begin{array}{c}\text { Forms the receptor site with Tom22 of } \\
\text { the major mitochondrial protein } \\
\text { importer. (TOMM) }\end{array}$ & $\downarrow$ \\
\hline Tom 40 & OMM & $\begin{array}{r}\text { Forms the channel portion of the } \\
\text { TOMM import complex }\end{array}$ & $\uparrow$ \\
\hline VDAC1 & OMM & Voltage dependent ion channel & $\uparrow$ \\
\hline SOD 2 & MM & $\begin{array}{r}\text { Mitochondrial specific neutralizer of } \\
\text { super oxide from electron transport } \\
\text { chain }\end{array}$ & $\downarrow$ \\
\hline PDH & MM & $\begin{array}{r}\text { Decarboxylates pyruvate to form } \\
\text { acetyl-CoA, a TCA cycle substrate. }\end{array}$ & $\downarrow$ \\
\hline IDH2 & MM & $\begin{array}{r}\text { NADP(+) dependent, catalyzes the } \\
\text { formation of } \alpha \text {-ketoglutarate from } \\
\text { isocitrate }\end{array}$ & $\uparrow$ \\
\hline IDH3 $\alpha$ & MM & $\begin{array}{r}\text { TCA cycle enzyme } \\
\text { NAD(+) dependent, catalyzes the } \\
\text { formation of } \alpha \text {-ketoglutarate from } \\
\text { isocitrate }\end{array}$ & $\downarrow$ \\
\hline Cytochrome C & IM & $\begin{array}{r}\text { Loosely associates with the IMM in the } \\
\text { IM. Serves was a carrier of electrons } \\
\text { from Complex III to Complex IV of the } \\
\text { electron transport chain }\end{array}$ & $\downarrow$ \\
\hline
\end{tabular}

\section{Table 2: Mitochondrial proteins analyzed using immunoblotting}

Mitochondrial proteins, their locations, functions, and relative changes in expression between cells depleted of SBDS and scrambled controls are shown. Changes in expression were determined by quantification with ImageJ software of band density normalized to a tubulin loading control using several different exposures for each blot and at least three replicate sample preparations in each cell type. $(\mathrm{OMM}=$ outer mitochondrial membrane, $\mathrm{MM}=$ mitochondrial matrix, $\mathrm{IM}=$ inner membrane of mitochondria) 

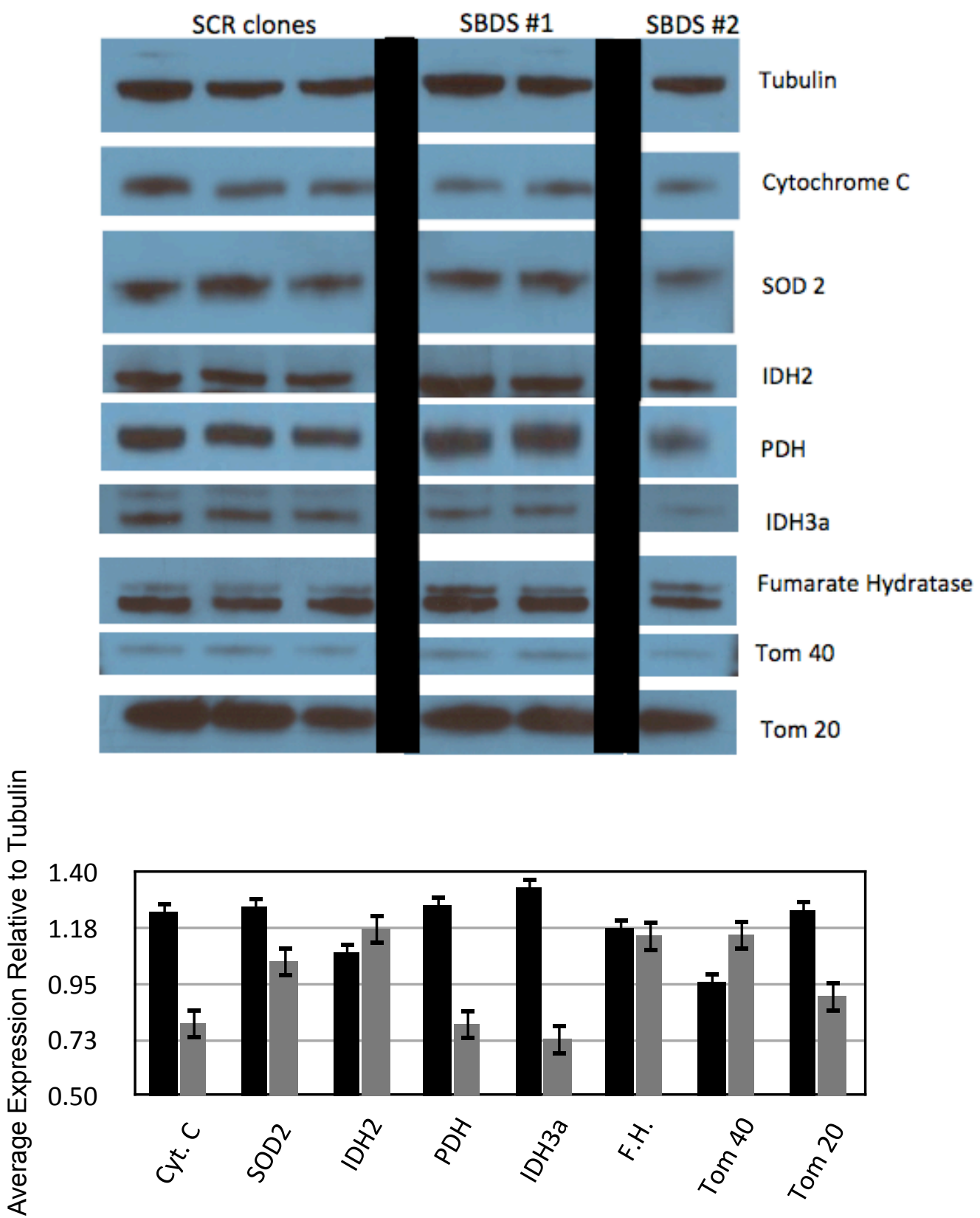

SCR SBDS

Fig 22: Steady state levels of representative mitochondrial proteins in cells depleted of SBDS relative to Scr controls.

Upper Panel: Immunoblots of whole cell lysates from TF-1 cells transduced with lentiviral vectors targeting SBDS gene expression or scrambled controls. (Black lines indicate a space in the membrane for protein size standard.) 
Lower Panel: Quantification of band intensity using ImageJ software. Each bar represents the average of multiple band intensities over multiple experiments and exposures relative to a tubulin loading control. Error bars = S.E.M.

(Cyt. C - Cytochrome C, SOD2 - superoxide dismutase 2, F.H.- Fumarate Hydratase) 

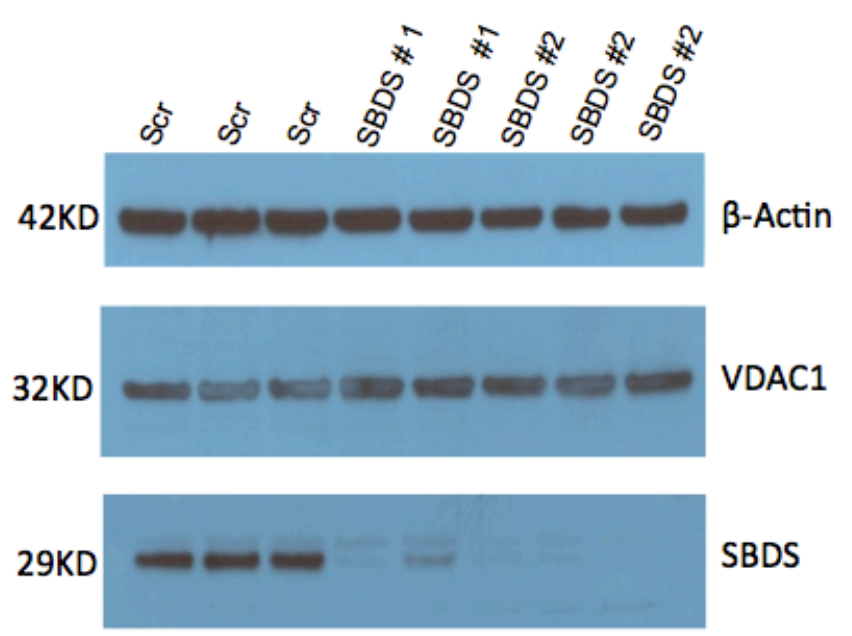

VDAC1 Expression

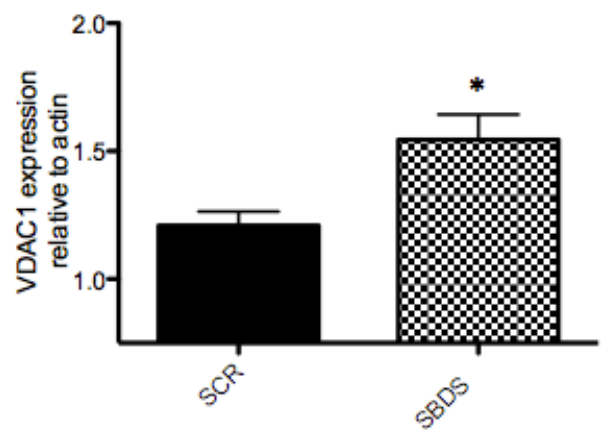

Fig 23: Analysis of VDAC1 expression in TF-1 cells depleted of SBDS compared with scrambled controls.

Upper panel: Cell extracts were fractionated by SDS-PAGE, transferred to Nitrocellulose and were immunoblotted with antibodies raised to VDAC1, SBDS, and $\beta$-actin. Lower panel: Quantification of band intensity from multiple blots show increase in VDAC1 expression with SBDS knockdown quantified using ImageJ software available from the NIH. Graph represents the mean with S.D. used for error bars. ( ${ }^{*} p<0.05$ as determined by an unpaired Student's t-test) 
Increased ROS production in cells depleted of SBDS

Decreases in mitochondrial function are often associated with an increased production of reactive oxygen species. To assess ROS production in our model system we employed 2',7'-dichlorfluorescein-diacetate (DCFH-DA). This compound is rapidly cleaved by cellular esterases to the non-fluorescent compound DCFH. DCFH, in turn, is oxidized to the highly fluorescent compound DCF in the presence of cellular peroxidases and ROS (Lebel and Bondy, 1990; Oyama et al., 1994; Rosenkranz et al., 1992; Trayner et al., 1995). Figure 24A shows that cells depleted of SBDS generate ROS at a rate significantly greater than that of scrambled controls. Furthermore, steady-state measurements indicated that cells depleted of SBDS had on average approximately double the amount of ROS compared to controls (Fig. 24B). 


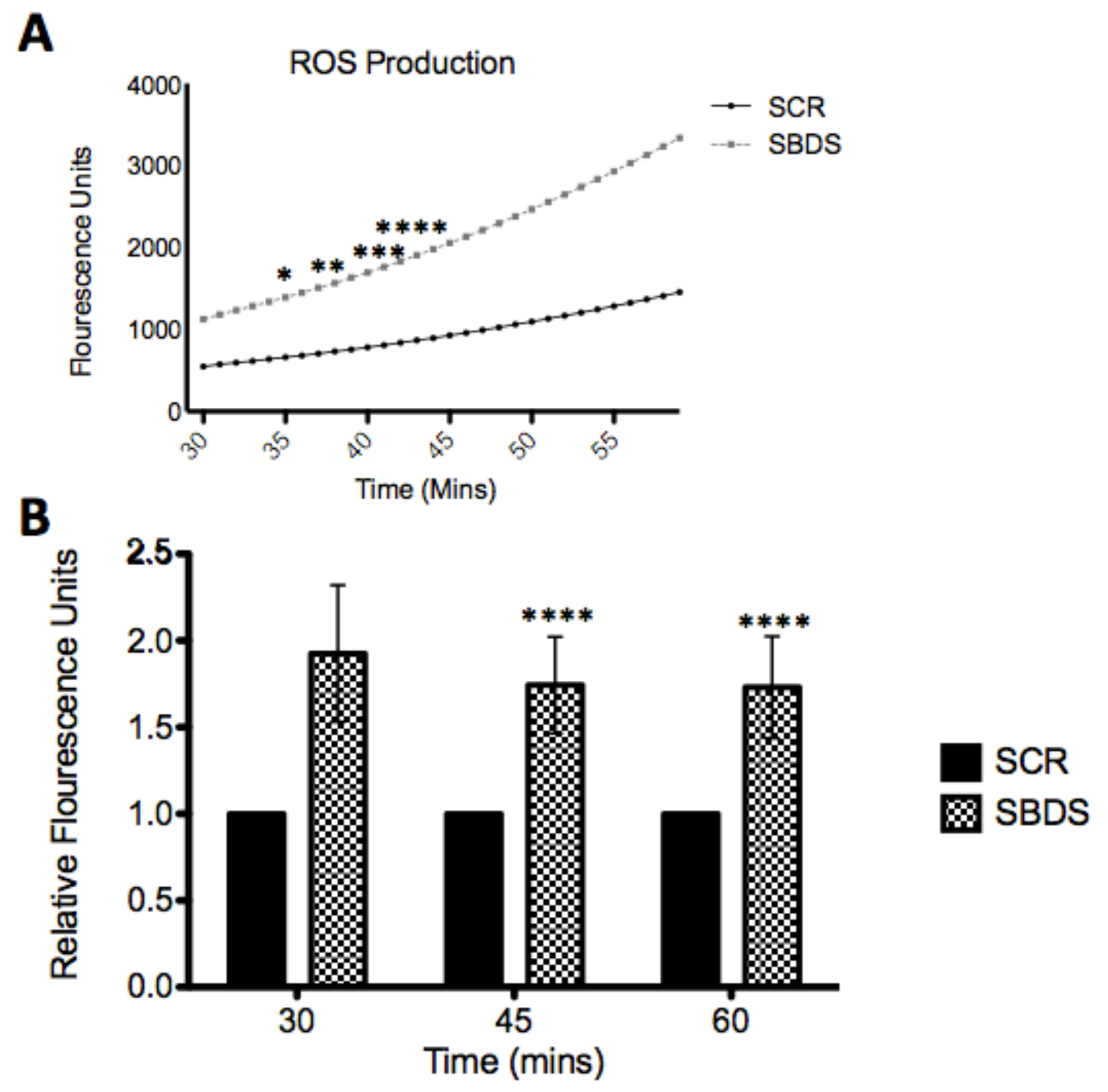

Fig 24: Analysis of ROS levels in TF-1 cells depleted of SBDS

(A) Representative fluorescence emission with time after treatment with $20 \mu \mathrm{M}$ DCFH-DA. (B) Data were plotted as relative fluorescence units in either TF-1 cells depleted of SBDS or scrambled controls. Values represent an average of three independent experiments. Error bars represent S.D. $\left({ }^{*} p<0.05\right.$ ${ }^{* *} p<0.01{ }^{* * *} p<0.001{ }^{* * * *} p<0.0001$, as determined by an unpaired student's ttest with earliest time point of specified variation labeled) 
Treatment with a reactive oxygen species scavenger in SDS models has been shown in prior works to reduce the amount of ROS created with SBDS knockdown samples (Ambekar et al., 2010). To determine if the enhanced ROS production in cells depleted of SBDS was limiting growth, we asked whether the anti-oxidant $\mathrm{N}$-acetyl-cysteine, (NAC), is capable of rescuing the growth deficit associated with loss of SBDS shown in Figure 16. Figure 25 shows that the addition of NAC had very complex effects in our tissue culture system. First and foremost, NAC appears to exhibit a dose dependent toxicity to scrambled control TF-1 cells. As there are no published studies examining the effects of NAC in TF-1 cells, it is unclear whether this observation is peculiar to our model system or is a general characteristic of TF-1 cells. In TF-1 cells depleted of SBDS this toxicity appears somewhat reduced, and at higher concentrations of NAC growth appears to be rescued (Fig. 25). 


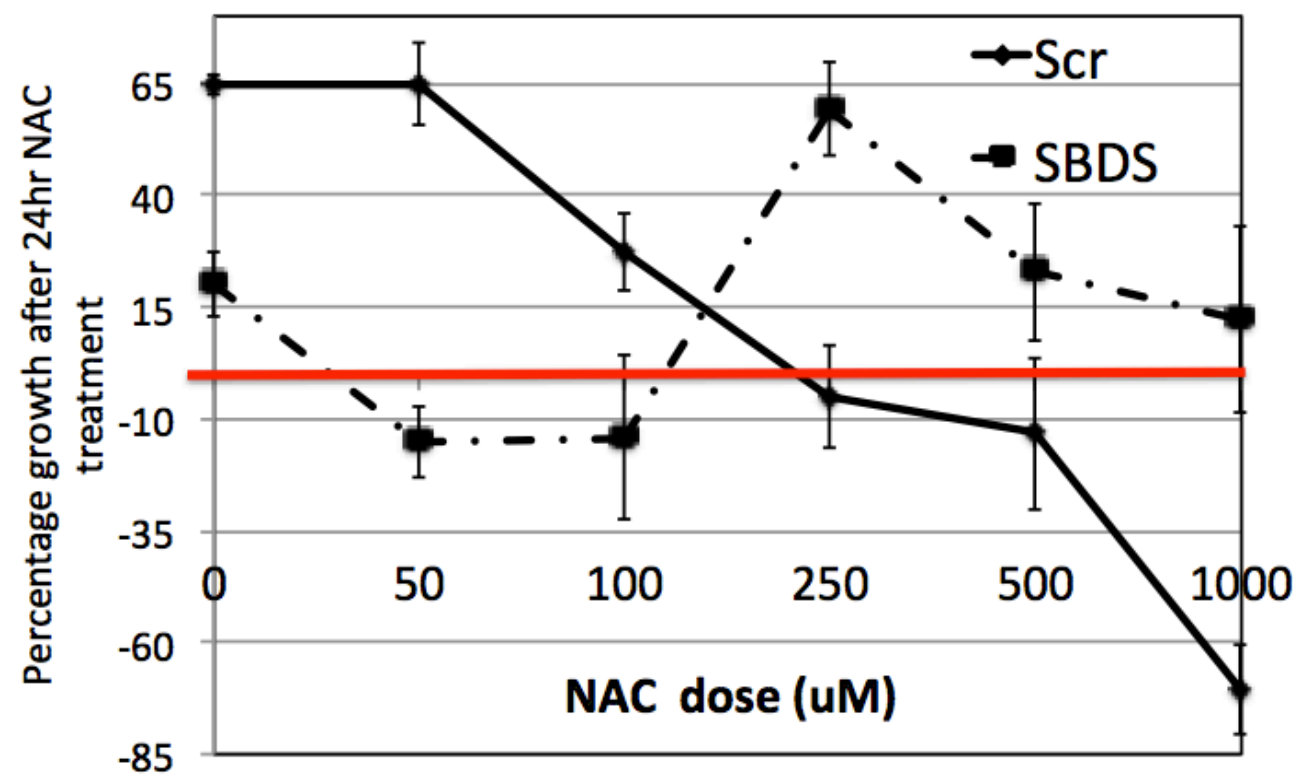

Fig. 25: Complex effects of NAC on growth and viability of TF-1 cell cultures.

TF-1 cells transduced with shRNA targeting SBDS or a scrambled control sequence were treated with the concentrations of NAC shown on the $\mathrm{x}$-axis. After 24 hours the samples were counted using trypan blue exclusion for cell viability. Values on the $y$-axis present the percentage of cell growth during the 24 hour immediately after treatment. Percentages were calculated by the count of viable cells after treatment divided by the pre-treatment number of cells multiplied by 100 . The numbers shown are the average of three independent experiments with error bars representing the standard deviation. The red line represents the $x$-axis at 0 , which represents an equal amount of cell growth and cell death in the 24 hour time frame. 


\section{Discussion}

We have developed a model of SDS that allows us to study aspects of disease pathophysiology in a human cell line of myeloid lineage. TF-1 erythroleukemic cells transduced with lentiviral vectors targeting SBDS gene expression were used in these experiments. These cell lines showed less than $30 \%$ of scrambled control levels of SBDS mRNA levels and nearly undetectable protein levels (Fig. 14). The low levels of SBDS observed in our system are a reasonable approximation to the situation in SDS patients where trace levels of SBDS are expressed from hypomorphic alleles in this autosomal recessive disorder (Boocock et al., 2003).

Our models system exhibited many of the hallmarks associated with reduced SBDS expression in a number of other systems. SBDS is known to function in ribosome biogenesis. In yeast models of SDS, SBDS is required for the release of the anti-association factor Tif6, the yeast ortholog of elF6, from fully matured $60 \mathrm{~S}$ subunits in the cytoplasm. The continued presence of Tif6/ elF6 on 605 subunits in the cytoplasm prevents their association with $40 \mathrm{~S}$ subunits in forming $80 \mathrm{~S}$ initiation complexes and so reduces the translational capacity of cells. The lack of recycling of Tif6/elF6 back to the nucleus also eventually leads to the nuclear accumulation of pre-60S subunits as Tif6/elF6 is also required for late steps in the maturation of 605 subunits in the nucleus (Menne et al., 2007; Moore et al., 2010). In our system the most apparent effect of the depletion of SBDS on the translational machinery is a reduction in polysomes consistent with a decrease in the number of functional ribosomes. 
Further we found that this decrease in translational capacity correlated with an increased presence of elF6 in the 60S subunit fraction.

Neutropenia is the most common blood lineage dysfunction seen in SDS with disease severity often relapsing and remitting over time and can often include other BM lineages (Burroughs et al., 2009). SDS predominantly affects cells of myleloid lineages which is why we turned to TF-1 cells in creating our cell-based model. TF-1 cells are able to undergo partial myeloid or erythroid differentiation when grown in either GM-CSF or EPO, respectively (Miyake et al., 2005). Our experiments demonstrated that depletion of SBDS reduced differentiation along both myeloid and erythroid lineages (Fig. 19). Other cell models have shown that SBDS is an important regulator of differentiation and proliferation of hematopoietic cells of both erythroid and granulocytic lineages, which is congruent with our findings and marrow phenotypes observed in SDS patients (Sen et al., 2011). In addition to the failure to differentiate along erythroid and granulocytes lineages, the TF-1 cell model reported here also shows a delay in cell cycle progression associated with decreased SBDS expression.

A major focus of our efforts with the TF-1 cell model of SDS was to determine if decreased expression of SBDS would interfere with mitochondrial function. These experiments were based, in part, on the observation that yeast cells depleted of Sdo1 were unable to grow on non-fermentable carbon sources. Another observation that directed us towards an assessment of mitochondrial function was a recent report indicating that mammalian cells depleted of SBDS 
showed an increase in ROS production. As mitochondria are a major source of ROS in many cells types and increases in ROS production is sensitive to mild uncoupling of the mitochondrial membrane potential it seemed reasonable to hypothesize that cells depleted of SBDS could have compromised mitochondrial function.

We employed two independent approaches to assess mitochondrial function in human TF-1 cells depleted of SBDS. The first was to measure oxygen consumption while the second was to evaluate mitochondrial membrane potential. The rates of basal and maximal oxygen consumption in SBDS knockdown cells were reduced relative to scrambled controls (Fig 20). The effect on basal oxygen consumption appeared greater when comparing the slopes of oxygen consumption curves before and after CCCP addition. The ratio in SBDS depleted samples was increased approximately $18 \%$ relative to scrambled controls suggesting there were affects on both mitochondrial mass and function. The finding that mitochondrial membrane potential also appeared lower in cells depleted of SBDS is consistent with an effect on mitochondrial function.

The work outlined here is the first to describe mitochondrial dysfunction in cells depleted of SBDS. Intriguingly however, these are not the first studies to link mitochondrial dysfunction to a bone marrow failure syndrome. Pearson's syndrome, which is caused by mutations in mitochondrial DNA, is characterized by anemia/pancytopenia and defective exocrine pancreas failure, reminiscent of the cardinal pathologies seen in SDS (Lee et al., 2007; Shimamura and Alter, 
2010; Topaloglu et al., 2008; Williams et al., 2012). Whether these two syndromes will converge to a common underlying pathological mechanism based on mitochondrial dysfunction remains to be seen.

We have examined the steady-state levels of a number of mitochondrial proteins to obtain a better perspective on changes brought about on mitochondrial function in cells depleted of SBDS. We have seen no consistent pattern of changes in expression of mitochondrial proteins involved in the organelle import machinery or in antioxidant defense enzymes in response to SBDS depletion. Two proteins in each of these classes were examined with one increasing expression and the other decreasing expression in both cases. One feature of this analysis which may be of note is the finding that proteins directly involved in energy metabolism, $\mathrm{PDH}, \mathrm{IDH} 3 \alpha$, and cytochrome c are all decreased in cells depleted of SBDS. A decrease in the activity of the TCA cycle, linked to reduced IDH3 $\alpha$ activity, or reduced acetyl-CoA as a result of decreased activity of $\mathrm{PDH}$, could have an impact on mitochondrial membrane potential as could lower amounts of cytochrome c.

Compromised function of the electron transport chain could in principle lead to an increase in ROS generation such as that observed now in two different models of SDS and in multiple models of altered cellular metabolism in carcinogenesis (Ahn et al., 2008; Bell et al., 2011; Bell and Guarente, 2011; Choudhary et al., 2009; Finley et al., 2011; Haigis et al., 2012; Hanahan and Weinberg, 2011; Huang et al., 2010; Kim et al., 2010; Schumacker, 2006). While at present this relationship is simply a correlation, it does represent a fruitful area 
of investigation. The finding that the antioxidant NAC rescues some of the growth characteristics of cells depleted of SBDS suggests that the increase in ROS production may be a contributing factors to SDS pathophysiology.

Finally, similar to what was observed in yeast we see an increase in the amount of VDAC1 in cells depleted of SBDS. Both increased and decreased expression of VDAC1 has been linked to apoptosis and so it is possible that changes in VDAC levels could potentially contribute to enhanced cell death in cellular models of SDS.

Changes in particular mitochondrial proteins, in addition to VDAC1, are reminiscent of other experiments performed in our lab and the works of others. Joseph Moore IV, formerly of the Ellis lab and in collaboration with researchers at Texas A \& M, performed a drug screen for rescue of the growth deficit of SDO1 yeast strains. They discovered that the HDAC inhibitor TSA could lead to global changes in protein expression and acetylation that mimicked the wild-type samples as well as rescuing the growth deficit. In other settings where global protein acetylation is affected by loss of a protein responsible for deacetylation, loss of SIRT3, a member of another HDAC family, in both mouse models and yeast strains, leads to changes in global protein acetylation (Ahn et al., 2008; Choudhary et al., 2009; Kim et al., 2010; Tao et al., 2010). It is one of a class of acetylation regulating enzymes called sirtuins, and this particular one is the major mitochondrial form that functions as an NAD-dependent de-acetylase. For example, the mitochondrial reactive oxygen species scavenger SOD2 is hyperacetylated and cannot perform its normal functions efficiently (Tao et al., 
2010). This prevents the mitochondria from dealing with normal concentrations of superoxide radicals while simultaneously in this system Hif1a, a hypoxia induced transcription factor, increases the expression of gene targets responsible for the so-called Warburg effect(Bell et al., 2011; Bell and Guarente, 2011; Finley et al., 2011). The Warburg effect is the mechanism by which cancerous cells preferentially metabolize glucose, even at the expense of generating large concentrations of reactive oxygen intermediates through inefficient oxidative respiration (Schumacker, 2006; Warburg, 1956). The acetylation status of multiple proteins, in addition to the changes in protein expression mediated by Hif1 $\alpha$, promote the reprogramming of cellular metabolism and the drive toward glycolysis as the sole energy producing pathway (Ahn et al., 2008; Choudhary et al., 2009; Finley et al., 2011; Haigis et al., 2012; Hanahan and Weinberg, 2011; Huang et al., 2010; Kim et al., 2010; Tao et al., 2010). One downstream modification is to decrease expression of SOD2. Thus, overall ROS levels are increased by two mechanisms, both an increase in the production of ROS from the increased activity of the electron transport chain given the cell's preference for metabolism of glucose, and a decrease in protein levels of SOD2 and the efficiency of super oxide radical scavenging of the individual SOD2 proteins due to hyper-acetylation (Ahn et al., 2008; Bell et al., 2011; Bell and Guarente, 2011; Finley et al., 2011; Haigis et al., 2012; Kim et al., 2010; Tao et al., 2010). There are questions left to answer in the complex interplay between SBDS, ROS, and the function of the mitochondria in SDS pathophysiology. 
Together, our findings point to mitochondria as a source of new physiologic functions for SBDS, which adds to its reported roles in ribosome synthesis, chemotaxis, and mitotic spindle assembly (Ambekar et al., 2010; Austin et al., 2008; Ball et al., 2009; Brina et al., 2011; Finch et al., 2011; Ganapathi et al., 2007; Leung et al., 2011; Nihrane et al., 2009; Orelio et al., 2011; Orelio et al., 2009; Rawls et al., 2007; Sen et al., 2011; Smith et al., 1996; Watanabe et al., 2009; Wong et al., 2011; Yamaguchi et al., 2011; Yamaguchi et al., 2007). 


\section{$\underline{\text { Conclusions and Future Directions }}$}

The complex role of SBDS in cellular metabolism and respiration is inevitably far more intricate than the scope of this dissertation work. These studies were initiated to follow up on the observation that yeast lacking Sdo1, the Sbds ortholog, were unable to grow on non-fermentable carbon sources indicating a defect in mitochondrial function. We used multiple approaches to study mitochondrial function in human cells depleted of SBDS, each of which were consistent with a role for SBDS in maintaining mitochondrial function. These studies present a new avenue of investigation into the pathophysiology of Shwachman Diamond syndrome. Moreover, they also present new opportunities for therapeutic intervention. 


\section{REFERENCES}

Ahn, B.H., Kim, H.S., Song, S., Lee, I.H., Liu, J., Vassilopoulos, A., Deng, C.X., and Finkel, T. (2008). A role for the mitochondrial deacetylase Sirt3 in regulating energy homeostasis. Proc Natl Acad Sci U S A 105, 14447-14452.

Aktipis, S., and Panayotatos, N. (1981). A kinetic study on the mechanism of inhibition of RNA synthesis catalyzed by DNA-dependent RNA polymerase. Differences in inhibition by ethidium bromide, 3,8-diamino-6ethylphenanthridinium bromide and actinomycin d. Biochimica et biophysica acta $655,278-290$.

Alexander, W.S., Roberts, A.W., Maurer, A.B., Nicola, N.A., Dunn, A.R., and Metcalf, D. (1996a). Studies of the c-Mpl thrombopoietin receptor through gene disruption and activation. Stem Cells 14 Suppl 1, 124-132.

Alexander, W.S., Roberts, A.W., Nicola, N.A., Li, R., and Metcalf, D. (1996b). Deficiencies in progenitor cells of multiple hematopoietic lineages and defective megakaryocytopoiesis in mice lacking the thrombopoietic receptor c-Mpl. Blood $87,2162-2170$. 
Allison, L.A., North, M.T., and Neville, L.A. (1995). Differential binding of oocytetype and somatic-type 5S rRNA to TFIIIA and ribosomal protein L5 in Xenopus oocytes: specialization for storage versus mobilization. Dev Biol 168, 284-295.

Alter, B.P. (2007). Diagnosis, genetics, and management of inherited bone marrow failure syndromes. Hematology Am Soc Hematol Educ Program, 29-39.

Alter, B.P., Giri, N., Savage, S.A., Peters, J.A., Loud, J.T., Leathwood, L., Carr, A.G., Greene, M.H., and Rosenberg, P.S. (2010). Malignancies and survival patterns in the National Cancer Institute inherited bone marrow failure syndromes cohort study. Br J Haematol 150, 179-188.

Alter, B.P., Giri, N., Savage, S.A., and Rosenberg, P.S. (2009). Cancer in dyskeratosis congenita. Blood 113, 6549-6557.

Alter, B.P., Rosenberg, P.S., and Brody, L.C. (2007). Clinical and molecular features associated with biallelic mutations in FANCD1/BRCA2. J Med Genet 44, $1-9$.

Ambekar, C., Das, B., Yeger, H., and Dror, Y. (2010). SBDS-deficiency results in deregulation of reactive oxygen species leading to increased cell death and decreased cell growth. Pediatr Blood Cancer 55, 1138-1144. 
Ameziane, N., Errami, A., Leveille, F., Fontaine, C., de Vries, Y., van Spaendonk, R.M., de Winter, J.P., Pals, G., and Joenje, H. (2008). Genetic subtyping of Fanconi anemia by comprehensive mutation screening. Hum Mutat 29, 159-166.

Andreassen, P.R., D'Andrea, A.D., and Taniguchi, T. (2004). ATR couples FANCD2 monoubiquitination to the DNA-damage response. Genes Dev 18, 1958-1963.

Austin, K.M., Gupta, M.L., Coats, S.A., Tulpule, A., Mostoslavsky, G., Balazs, A.B., Mulligan, R.C., Daley, G., Pellman, D., and Shimamura, A. (2008). Mitotic spindle destabilization and genomic instability in Shwachman-Diamond syndrome. J Clin Invest 118, 1511-1518.

Austin, K.M., Leary, R.J., and Shimamura, A. (2005). The Shwachman-Diamond SBDS protein localizes to the nucleolus. Blood 106, 1253-1258.

Badhai, J., Frojmark, A.S., Razzaghian, H.R., Davey, E., Schuster, J., and Dahl, N. (2009). Posttranscriptional down-regulation of small ribosomal subunit proteins correlates with reduction of 18S rRNA in RPS19 deficiency. FEBS Lett $583,2049-2053$.

Baerlocher, K.E., Feldges, A., Weissert, M., Simonsz, H.J., and Rotig, A. (1992). Mitochondrial DNA deletion in an 8-year-old boy with Pearson syndrome. Journal of inherited metabolic disease 15, 327-330. 
Bakker, S.T., van de Vrugt, H.J., Rooimans, M.A., Oostra, A.B., Steltenpool, J., Delzenne-Goette, E., van der Wal, A., van der Valk, M., Joenje, H., te Riele, H., et al. (2009). Fancm-deficient mice reveal unique features of Fanconi anemia complementation group M. Hum Mol Genet 18, 3484-3495.

Ball, H.L., Zhang, B., Riches, J.J., Gandhi, R., Li, J., Rommens, J.M., and Myers, J.S. (2009). Shwachman-Bodian Diamond syndrome is a multi-functional protein implicated in cellular stress responses. Hum Mol Genet 18, 3684-3695.

Ball, S. (2011). Diamond Blackfan anemia. Hematology Am Soc Hematol Educ Program 2011, 487-491.

Bartley, T.D., Bogenberger, J., Hunt, P., Li, Y.S., Lu, H.S., Martin, F., Chang, M.S., Samal, B., Nichol, J.L., Swift, S., et al. (1994). Identification and cloning of a megakaryocyte growth and development factor that is a ligand for the cytokine receptor Mpl. Cell 77, 1117-1124.

Basu, U., Si, K., Warner, J.R., and Maitra, U. (2001). The Saccharomyces cerevisiae TIF6 gene encoding translation initiation factor 6 is required for $60 \mathrm{~S}$ ribosomal subunit biogenesis. Mol Cell Biol 21, 1453-1462.

Bell, E.L., Emerling, B.M., Ricoult, S.J., and Guarente, L. (2011). SirT3 suppresses hypoxia inducible factor 1 alpha and tumor growth by inhibiting mitochondrial ROS production. Oncogene 30, 2986-2996. 
Bell, E.L., and Guarente, L. (2011). The SirT3 divining rod points to oxidative stress. Mol Cell 42, 561-568.

Ben-Shem, A., Jenner, L., Yusupova, G., and Yusupov, M. (2010). Crystal structure of the eukaryotic ribosome. Science 330, 1203-1209.

Berthou, C., Devergie, A., D'Agay, M.F., Sonsino, E., Scrobohaci, M.L., Loirat, C., and Gluckman, E. (1991). Late vascular complications after bone marrow transplantation for dyskeratosis congenita. $\mathrm{Br} \mathrm{J}$ Haematol 79, 335-336.

Bianco, P. (2011). Minireview: The stem cell next door: skeletal and hematopoietic stem cell "niches" in bone. Endocrinology 152, 2957-2962.

Blatt, J., Katerji, A., Barmada, M., Wenger, S.L., and Penchansky, L. (1994). Pancytopenia and vacuolation of marrow precursors associated with necrotizing encephalopathy. Br J Haematol 86, 207-209.

Blaw, M.E., and Mize, C.E. (1990). Juvenile Pearson syndrome. Journal of child neurology $5,187-190$.

Boocock, G.R., Morrison, J.A., Popovic, M., Richards, N., Ellis, L., Durie, P.R., and Rommens, J.M. (2003). Mutations in SBDS are associated with Shwachman-Diamond syndrome. Nat Genet 33, 97-101. 
Boria, I., Garelli, E., Gazda, H.T., Aspesi, A., Quarello, P., Pavesi, E., Ferrante, D., Meerpohl, J.J., Kartal, M., Da Costa, L., et al. (2010). The ribosomal basis of Diamond-Blackfan Anemia: mutation and database update. Hum Mutat 31, 1269-1279.

Boultwood, J., Pellagatti, A., and Wainscoat, J.S. (2011). Haploinsufficiency of ribosomal proteins and p53 activation in anemia: Diamond-Blackfan anemia and the $5 q$ - syndrome. Advances in enzyme regulation.

Brina, D., Grosso, S., Miluzio, A., and Biffo, S. (2011). Translational control by $80 S$ formation and 60S availability: the central role of elF6, a rate limiting factor in cell cycle progression and tumorigenesis. Cell Cycle 10, 3441-3446.

Burroughs, L., Woolfrey, A., and Shimamura, A. (2009). Shwachman-Diamond syndrome: a review of the clinical presentation, molecular pathogenesis, diagnosis, and treatment. Hematology/oncology clinics of North America 23, 233-248.

Burwick, N., Shimamura, A., and Liu, J.M. (2011). Non-Diamond Blackfan anemia disorders of ribosome function: Shwachman Diamond syndrome and 5qsyndrome. Semin Hematol 48, 136-143.

Campagnoli, M.F., Ramenghi, U., Armiraglio, M., Quarello, P., Garelli, E., Carando, A., Avondo, F., Pavesi, E., Fribourg, S., Gleizes, P.E., et al. (2008). 
RPS19 mutations in patients with Diamond-Blackfan anemia. Hum Mutat 29, 911-920.

Campian, J.L., Gao, X., Qian, M., and Eaton, J.W. (2007). Cytochrome C oxidase activity and oxygen tolerance. J Biol Chem 282, 12430-12438.

Carradice, D., and Lieschke, G.J. (2008). Zebrafish in hematology: sushi or science? Blood 111, 3331-3342.

Casse, C., Giannoni, F., Nguyen, V.T., Dubois, M.F., and Bensaude, O. (1999). The transcriptional inhibitors, actinomycin D and alpha-amanitin, activate the HIV-1 promoter and favor phosphorylation of the RNA polymerase II C-terminal domain. J Biol Chem 274, 16097-16106.

Cavazzana-Calvo, M., Fischer, A., Bushman, F.D., Payen, E., Hacein-Bey-Abina, S., and Leboulch, P. (2011). Is normal hematopoiesis maintained solely by longterm multipotent stem cells? Blood 117, 4420-4424.

Cech, T.R. (2000). Structural biology. The ribosome is a ribozyme. Science 289, 878-879.

Chacko, A.D., Liberante, F., Paul, I., Longley, D.B., and Fennell, D.A. (2010). Voltage dependent anion channel-1 regulates death receptor mediated apoptosis by enabling cleavage of caspase-8. BMC cancer 10, 380 . 
Chaoui, D., Faussat, A.M., Majdak, P., Tang, R., Perrot, J.Y., Pasco, S., Klein, C., Marie, J.P., and Legrand, O. (2006). JC-1, a sensitive probe for a simultaneous detection of P-glycoprotein activity and apoptosis in leukemic cells. Cytometry. Part B, Clinical cytometry 70, 189-196.

Chazotte, B. (2011). Labeling mitochondria with JC-1. Cold Spring Harbor protocols 2011.

Chen, D., Zhang, Z., Li, M., Wang, W., Li, Y., Rayburn, E.R., Hill, D.L., Wang, H., and Zhang, R. (2007). Ribosomal protein S7 as a novel modulator of p53-MDM2 interaction: binding to MDM2, stabilization of p53 protein, and activation of p53 function. Oncogene 26, 5029-5037.

Choesmel, V., Bacqueville, D., Rouquette, J., Noaillac-Depeyre, J., Fribourg, S., Cretien, A., Leblanc, T., Tchernia, G., Da Costa, L., and Gleizes, P.E. (2007). Impaired ribosome biogenesis in Diamond-Blackfan anemia. Blood 109, $1275-1283$.

Choesmel, V., Fribourg, S., Aguissa-Toure, A.H., Pinaud, N., Legrand, P., Gazda, H.T., and Gleizes, P.E. (2008). Mutation of ribosomal protein RPS24 in DiamondBlackfan anemia results in a ribosome biogenesis disorder. Hum Mol Genet 17, $1253-1263$.

Choi, K. (1998). Hemangioblast development and regulation. Biochemistry and cell biology $=$ Biochimie et biologie cellulaire 76, 947-956 . 
Choudhary, C., Kumar, C., Gnad, F., Nielsen, M.L., Rehman, M., Walther, T.C., Olsen, J.V., and Mann, M. (2009). Lysine acetylation targets protein complexes and co-regulates major cellular functions. Science 325, 834-840.

Ciganda, M., and Williams, N. (2011). Eukaryotic 5S rRNA biogenesis. Wiley interdisciplinary reviews. RNA 2, 523-533.

Clegg, H.V., Itahana, K., and Zhang, Y. (2008). Unlocking the Mdm2-p53 loop: ubiquitin is the key. Cell Cycle 7, 287-292.

Collins, T.E. (1900). Case with Symmetrical congenital notches in the outer part of each lower lid and defective development of the malar bones. Trans Opthalmol Soc UK 20.

Cormier, V., Rotig, A., Quartino, A.R., Forni, G.L., Cerone, R., Maier, M., Saudubray, J.M., and Munnich, A. (1990). Widespread multi-tissue deletions of the mitochondrial genome in the Pearson marrow-pancreas syndrome. J Pediatr 117, 599-602.

Cossarizza, A., and Salvioli, S. (2001). Flow cytometric analysis of mitochondrial membrane potential using JC-1. Current protocols in cytometry / editorial board, J. Paul Robinson, managing editor ... [et al.] Chapter 9, Unit 914. 
Cotter, P.D., Baumann, M., and Bishop, D.F. (1992). Enzymatic defect in "Xlinked" sideroblastic anemia: molecular evidence for erythroid deltaaminolevulinate synthase deficiency. Proc Natl Acad Sci U S A 89, 4028-4032.

Cotter, P.D., Rucknagel, D.L., and Bishop, D.F. (1994). X-linked sideroblastic anemia: identification of the mutation in the erythroid-specific deltaaminolevulinate synthase gene (ALAS2) in the original family described by Cooley. Blood 84, 3915-3924.

Dai, M.S., Arnold, H., Sun, X.X., Sears, R., and Lu, H. (2007a). Inhibition of cMyc activity by ribosomal protein L11. EMBO J 26, 3332-3345.

Dai, M.S., and Lu, H. (2004). Inhibition of MDM2-mediated p53 ubiquitination and degradation by ribosomal protein L5. J Biol Chem 279, 44475-44482.

Dai, M.S., and Lu, H. (2008). Crosstalk between c-Myc and ribosome in ribosomal biogenesis and cancer. J Cell Biochem 105, 670-677.

Dai, M.S., Sears, R., and Lu, H. (2007b). Feedback regulation of c-Myc by ribosomal protein L11. Cell Cycle 6, 2735-2741.

Dai, M.S., Shi, D., Jin, Y., Sun, X.X., Zhang, Y., Grossman, S.R., and Lu, H. (2006). Regulation of the MDM2-p53 pathway by ribosomal protein L11 involves a post-ubiquitination mechanism. J Biol Chem 281, 24304-24313. 
Dai, M.S., Sun, X.X., and Lu, H. Ribosomal protein L11 associates with c-Myc at $5 \mathrm{~S}$ rRNA and tRNA genes and regulates their expression. J Biol Chem 285, 12587-12594.

Dai, M.S., Zeng, S.X., Jin, Y., Sun, X.X., David, L., and Lu, H. (2004). Ribosomal protein L23 activates p53 by inhibiting MDM2 function in response to ribosomal perturbation but not to translation inhibition. Mol Cell Biol 24, 7654-7668.

Dale, D.C., Bolyard, A.A., Schwinzer, B.G., Pracht, G., Bonilla, M.A., Boxer, L., Freedman, M.H., Donadieu, J., Kannourakis, G., Alter, B.P., et al. (2006). The Severe Chronic Neutropenia International Registry: 10-Year Follow-up Report. Supportive cancer therapy 3, 220-231.

Dale, D.C., Person, R.E., Bolyard, A.A., Aprikyan, A.G., Bos, C., Bonilla, M.A., Boxer, L.A., Kannourakis, G., Zeidler, C., Welte, K., et al. (2000). Mutations in the gene encoding neutrophil elastase in congenital and cyclic neutropenia. Blood 96, 2317-2322.

Danilova, N., Sakamoto, K.M., and Lin, S. (2008). Ribosomal protein S19 deficiency in zebrafish leads to developmental abnormalities and defective erythropoiesis through activation of p53 protein family. Blood 112, 5228-5237.

de Sauvage, F.J., Hass, P.E., Spencer, S.D., Malloy, B.E., Gurney, A.L., Spencer, S.A., Darbonne, W.C., Henzel, W.J., Wong, S.C., Kuang, W.J., et al. (1994). 
Stimulation of megakaryocytopoiesis and thrombopoiesis by the c-Mpl ligand. Nature 369, 533-538.

De Stefani, D., Bononi, A., Romagnoli, A., Messina, A., De Pinto, V., Pinton, P., and Rizzuto, R. (2011). VDAC1 selectively transfers apoptotic $\mathrm{Ca}(2+)$ signals to mitochondria. Cell Death Differ.

de Winter, J.P., and Joenje, H. (2009). The genetic and molecular basis of Fanconi anemia. Mutation research 668, 11-19.

Dempster A, R.D. (1977). Maximum likelihood from incomplete data via the EM algorithm. Journal of the Royal Statistical Society, series B - Methodoligical 39, 1-38.

Devlin, E.E., Dacosta, L., Mohandas, N., Elliott, G., and Bodine, D.M. A transgenic mouse model demonstrates a dominant negative effect of a point mutation in the RPS19 gene associated with Diamond-Blackfan anemia. Blood $116,2826-2835$.

Dixon, J., Jones, N.C., Sandell, L.L., Jayasinghe, S.M., Crane, J., Rey, J.P., Dixon, M.J., and Trainor, P.A. (2006). Tcof1/Treacle is required for neural crest cell formation and proliferation deficiencies that cause craniofacial abnormalities. Proc Natl Acad Sci U S A 103, 13403-13408. 
Dixon S. J.E, Gladwin A, Dixon M, Loftus S., Bonner, C., Koprivnikar K., Wasthmuth J (1996). Positional cloning of a gene involved in the pathogenesis of Treacher Collins syndrome. Nat Genet 12, 130-136.

Donadieu, J., Michel, G., Merlin, E., Bordigoni, P., Monteux, B., Beaupain, B., Leverger, G., Laporte, J.P., Hermine, O., Buzyn, A., et al. (2005). Hematopoietic stem cell transplantation for Shwachman-Diamond syndrome: experience of the French neutropenia registry. Bone Marrow Transplant 36, 787-792.

Dragon, F., Gallagher, J.E., Compagnone-Post, P.A., Mitchell, B.M., Porwancher, K.A., Wehner, K.A., Wormsley, S., Settlage, R.E., Shabanowitz, J., Osheim, Y., et al. (2002). A large nucleolar U3 ribonucleoprotein required for $18 \mathrm{~S}$ ribosomal RNA biogenesis. Nature 417, 967-970.

Draptchinskaia, N., Gustavsson, P., Andersson, B., Pettersson, M., Willig, T.N., Dianzani, I., Ball, S., Tchernia, G., Klar, J., Matsson, H., et al. (1999). The gene encoding ribosomal protein $\mathrm{S} 19$ is mutated in Diamond-Blackfan anaemia. Nat Genet 21, 169-175.

Dror, Y., and Freedman, M.H. (1999). Shwachman-Diamond syndrome: An inherited preleukemic bone marrow failure disorder with aberrant hematopoietic progenitors and faulty marrow microenvironment. Blood 94, 3048-3054.

Dror, Y., and Freedman, M.H. (2002). Shwachman-diamond syndrome. Br J Haematol 118, 701-713. 
Du, H.Y., Pumbo, E., Ivanovich, J., An, P., Maziarz, R.T., Reiss, U.M., Chirnomas, D., Shimamura, A., Vlachos, A., Lipton, J.M., et al. (2009). TERC and TERT gene mutations in patients with bone marrow failure and the significance of telomere length measurements. Blood 113, 309-316.

Ebert, B.L., Pretz, J., Bosco, J., Chang, C.Y., Tamayo, P., Galili, N., Raza, A., Root, D.E., Attar, E., Ellis, S.R., et al. (2008). Identification of RPS14 as a 5qsyndrome gene by RNA interference screen. Nature 451, 335-339.

Fagerlie, S., Lensch, M.W., Pang, Q., and Bagby, G.C., Jr. (2001). The Fanconi anemia group $\mathrm{C}$ gene product: signaling functions in hematopoietic cells. Exp Hematol 29, 1371-1381.

Farrar, J.E., and Dahl, N. (2011). Untangling the phenotypic heterogeneity of Diamond Blackfan anemia. Semin Hematol 48, 124-135.

Farrar, J.E., Nater, M., Caywood, E., McDevitt, M.A., Kowalski, J., Takemoto, C.M., Talbot, C.C., Jr., Meltzer, P., Esposito, D., Beggs, A.H., et al. (2008). Abnormalities of the large ribosomal subunit protein, Rpl35a, in DiamondBlackfan anemia. Blood 112, 1582-1592.

Farrar, J.E., Vlachos, A., Atsidaftos, E., Carlson-Donohoe, H., Markello, T.C., Arceci, R.J., Ellis, S.R., Lipton, J.M., and Bodine, D.M. (2011). Ribosomal protein gene deletions in Diamond-Blackfan anemia. Blood 118, 6943-6951. 
Fernandez, J., Bode, B., Koromilas, A., Diehl, J.A., Krukovets, I., Snider, M.D., and Hatzoglou, M. (2002). Translation mediated by the internal ribosome entry site of the cat-1 mRNA is regulated by glucose availability in a PERK kinasedependent manner. J Biol Chem 277, 11780-11787.

Ferreira, G.C. (1993). Erythroid 5-aminolevulinate synthase and X-linked sideroblastic anemia. The Journal of the Florida Medical Association 80, 481-483.

Finch, A.J., Hilcenko, C., Basse, N., Drynan, L.F., Goyenechea, B., Menne, T.F., Gonzalez Fernandez, A., Simpson, P., D'Santos, C.S., Arends, M.J., et al. (2011). Uncoupling of GTP hydrolysis from elF6 release on the ribosome causes Shwachman-Diamond syndrome. Genes Dev 25, 917-929.

Finley, L.W., Carracedo, A., Lee, J., Souza, A., Egia, A., Zhang, J., TeruyaFeldstein, J., Moreira, P.I., Cardoso, S.M., Clish, C.B., et al. (2011). SIRT3 opposes reprogramming of cancer cell metabolism through HIF1alpha destabilization. Cancer Cell 19, 416-428.

Flygare, J., Aspesi, A., Bailey, J.C., Miyake, K., Caffrey, J.M., Karlsson, S., and Ellis, S.R. (2007). Human RPS19, the gene mutated in Diamond-Blackfan anemia, encodes a ribosomal protein required for the maturation of $40 \mathrm{~S}$ ribosomal subunits. Blood 109, 980-986. 
Flygare, J., Kiefer, T., Miyake, K., Utsugisawa, T., Hamaguchi, I., Da Costa, L., Richter, J., Davey, E.J., Matsson, H., Dahl, N., et al. (2005). Deficiency of ribosomal protein S19 in CD34+ cells generated by siRNA blocks erythroid development and mimics defects seen in Diamond-Blackfan anemia. Blood 105, 4627-4634.

Fox, M.H. (1980). A model for the computer analysis of synchronous DNA distributions obtained by flow cytometry. Cytometry 1, 71-77.

Fromont-Racine, M., Senger, B., Saveanu, C., and Fasiolo, F. (2003). Ribosome assembly in eukaryotes. Gene $313,17-42$.

Fumagalli, S., Di Cara, A., Neb-Gulati, A., Natt, F., Schwemberger, S., Hall, J., Babcock, G.F., Bernardi, R., Pandolfi, P.P., and Thomas, G. (2009). Absence of nucleolar disruption after impairment of $40 \mathrm{~S}$ ribosome biogenesis reveals an rpL11-translation-dependent mechanism of p53 induction. Nat Cell Biol.

Gadalla, S.M., Cawthon, R., Giri, N., Alter, B.P., and Savage, S.A. (2010). Telomere length in blood, buccal cells, and fibroblasts from patients with inherited bone marrow failure syndromes. Aging 2, 867-874.

Galganska, H., Budzinska, M., Wojtkowska, M., and Kmita, H. (2008). Redox regulation of protein expression in Saccharomyces cerevisiae mitochondria: possible role of VDAC. Arch Biochem Biophys 479, 39-45. 
Galganska, H., Karachitos, A., Wojtkowska, M., Stobienia, O., Budzinska, M., and Kmita, H. (2010). Communication between mitochondria and nucleus: putative role for VDAC in reduction/oxidation mechanism. Biochimica et biophysica acta 1797, $1276-1280$.

Ganapathi, K.A., Austin, K.M., Lee, C.S., Dias, A., Malsch, M.M., Reed, R., and Shimamura, A. (2007). The human Shwachman-Diamond syndrome protein, SBDS, associates with ribosomal RNA. Blood 110, 1458-1465.

Ganapathi, K.A., and Shimamura, A. (2008). Ribosomal dysfunction and inherited marrow failure. $\mathrm{Br} \mathrm{J}$ Haematol 141, 376-387.

Gandin, V., Miluzio, A., Barbieri, A.M., Beugnet, A., Kiyokawa, H., Marchisio, P.C., and Biffo, S. (2008). Eukaryotic initiation factor 6 is rate-limiting in translation, growth and transformation. Nature 455, 684-688.

Gao, X., Qian, M., Campian, J.L., Marshall, J., Zhou, Z., Roberts, A.M., Kang, Y.J., Prabhu, S.D., Sun, X.F., and Eaton, J.W. (2010). Mitochondrial dysfunction may explain the cardiomyopathy of chronic iron overload. Free radical biology \& medicine 49, 401-407.

Garner, D.L., and Thomas, C.A. (1999). Organelle-specific probe JC-1 identifies membrane potential differences in the mitochondrial function of bovine sperm. Molecular reproduction and development 53, 222-229. 
Gavin, A.C., Bosche, M., Krause, R., Grandi, P., Marzioch, M., Bauer, A., Schultz, J., Rick, J.M., Michon, A.M., Cruciat, C.M., et al. (2002). Functional organization of the yeast proteome by systematic analysis of protein complexes. Nature 415 , $141-147$.

Gazda, H.T., Preti, M., Sheen, M.R., O'Donohue, M.F., Vlachos, A., Davies, S.M., Kattamis, A., Doherty, L., Landowski, M., Buros, C., et al. (2012). Frameshift mutation in p53 regulator RPL26 is associated with multiple physical abnormalities and a specific pre-ribosomal RNA processing defect in diamondblackfan anemia. Hum Mutat 33, 1037-1044.

Gazda, H.T., Sheen, M.R., Vlachos, A., Choesmel, V., O'Donohue, M.F., Schneider, H., Darras, N., Hasman, C., Sieff, C.A., Newburger, P.E., et al. (2008). Ribosomal protein L5 and L11 mutations are associated with cleft palate and abnormal thumbs in Diamond-Blackfan anemia patients. Am J Hum Genet 83, 769-780.

Gazda, H.T., Zhong, R., Long, L., Niewiadomska, E., Lipton, J.M., Ploszynska, A., Zaucha, J.M., Vlachos, A., Atsidaftos, E., Viskochil, D.H., et al. (2004). RNA and protein evidence for haplo-insufficiency in Diamond-Blackfan anaemia patients with RPS19 mutations. Br J Haematol 127, 105-113.

Germeshausen, M., Kratz, C.P., Ballmaier, M., and Welte, K. (2009a). RAS and CSF3R mutations in severe congenital neutropenia. Blood 114, 3504-3505. 
Germeshausen, M., Welte, K., and Ballmaier, M. (2009b). In vivo expansion of cells expressing acquired CSF3R mutations in patients with severe congenital neutropenia. Blood 113, 668-670.

Geula, S., Naveed, H., Liang, J., and Shoshan-Barmatz, V. (2011). StructureBased Analysis of Vdac1: Defining Oligomer Contact Sites. J Biol Chem.

Grandi, P., Rybin, V., Bassler, J., Petfalski, E., Strauss, D., Marzioch, M., Schafer, T., Kuster, B., Tschochner, H., Tollervey, D., et al. (2002). 90S pre-ribosomes include the $35 \mathrm{~S}$ pre-rRNA, the U3 snoRNP, and 40S subunit processing factors but predominantly lack 60 S synthesis factors. Mol Cell 10, 105-115.

Grenda, D.S., Murakami, M., Ghatak, J., Xia, J., Boxer, L.A., Dale, D., Dinauer, M.C., and Link, D.C. (2007). Mutations of the ELA2 gene found in patients with severe congenital neutropenia induce the unfolded protein response and cellular apoptosis. Blood 110, 4179-4187.

Gurney, A.L., Carver-Moore, K., de Sauvage, F.J., and Moore, M.W. (1994). Thrombocytopenia in c-mpl-deficient mice. Science 265, 1445-1447.

Guthrie, H.D., and Welch, G.R. (2008). Determination of high mitochondrial membrane potential in spermatozoa loaded with the mitochondrial probe 5,5', 6,6'-tetrachloro-1,1',3,3'-tetraethylbenzimidazolyl-carbocyanine iodide (JC-1) by using fluorescence-activated flow cytometry. Methods Mol Biol 477, 89-97. 
Hadjiolova, K.V., Nicoloso, M., Mazan, S., Hadjiolov, A.A., and Bachellerie, J.P. (1993). Alternative pre-rRNA processing pathways in human cells and their alteration by cycloheximide inhibition of protein synthesis. Eur J Biochem 212, 211-215.

Haigis, M.C., Deng, C.X., Finley, L.W., Kim, H.S., and Gius, D. (2012). SIRT3 Is a Mitochondrial Tumor Suppressor: A Scientific Tale That Connects Aberrant Cellular ROS, the Warburg Effect, and Carcinogenesis. Cancer research 72, 2468-2472.

Hanahan, D., and Weinberg, R.A. (2011). Hallmarks of cancer: the next generation. Cell 144, 646-674.

Heiss, N.S., Knight, S.W., Vulliamy, T.J., Klauck, S.M., Wiemann, S., Mason, P.J., Poustka, A., and Dokal, I. (1998). X-linked dyskeratosis congenita is caused by mutations in a highly conserved gene with putative nucleolar functions. Nat Genet 19, 32-38.

Honda, R., Tanaka, H., and Yasuda, H. (1997). Oncoprotein MDM2 is a ubiquitin ligase E3 for tumor suppressor p53. FEBS Lett 420, 25-27.

Horn, H.F., and Vousden, K.H. (2008). Cooperation between the ribosomal proteins L5 and L11 in the p53 pathway. Oncogene 27, 5774-5784. 
Horos, R., ljspeert, H., Pospisilova, D., Sendtner, R., Andrieu-Soler, C., Taskesen, E., Nieradka, A., Cmejla, R., Sendtner, M., Touw, I.P., et al. (2012). Ribosomal deficiencies in Diamond-Blackfan anemia impair translation of transcripts essential for differentiation of murine and human erythroblasts. Blood $119,262-272$.

Huang, J.Y., Hirschey, M.D., Shimazu, T., Ho, L., and Verdin, E. (2010). Mitochondrial sirtuins. Biochimica et biophysica acta 1804, 1645-1651.

Hurt, E., Hannus, S., Schmelzl, B., Lau, D., Tollervey, D., and Simos, G. (1999). A novel in vivo assay reveals inhibition of ribosomal nuclear export in ran-cycle and nucleoporin mutants. The Journal of cell biology $144,389-401$.

Ihara, K., Ishii, E., Eguchi, M., Takada, H., Suminoe, A., Good, R.A., and Hara, T. (1999). Identification of mutations in the c-mpl gene in congenital amegakaryocytic thrombocytopenia. Proc Natl Acad Sci U S A 96, 3132-3136.

Jaako, P., Flygare, J., Olsson, K., Quere, R., Ehinger, M., Henson, A., Ellis, S., Schambach, A., Baum, C., Richter, J., et al. (2011). Mice with ribosomal protein S19 deficiency develop bone marrow failure and symptoms like patients with Diamond-Blackfan anemia. Blood 118, 6087-6096.

Jackson, R.J., Hellen, C.U., and Pestova, T.V. (2010). The mechanism of eukaryotic translation initiation and principles of its regulation. Nature reviews. Molecular cell biology 11, 113-127. 
Jing, L., and Zon, L.I. (2011). Zebrafish as a model for normal and malignant hematopoiesis. Disease models \& mechanisms 4, 433-438.

Johnson, A.W., and Ellis, S.R. (2011). Of blood, bones, and ribosomes: is Swachman-Diamond syndrome a ribosomopathy? Genes Dev 25, 898-900.

Jones, N.C., Lynn, M.L., Gaudenz, K., Sakai, D., Aoto, K., Rey, J.P., Glynn, E.F., Ellington, L., Du, C., Dixon, J., et al. (2008). Prevention of the neurocristopathy Treacher Collins syndrome through inhibition of p53 function. Nat Med 14, 125-133.

Kaushansky, K., Lok, S., Holly, R.D., Broudy, V.C., Lin, N., Bailey, M.C., Forstrom, J.W., Buddle, M.M., Oort, P.J., Hagen, F.S., et al. (1994). Promotion of megakaryocyte progenitor expansion and differentiation by the c-Mpl ligand thrombopoietin. Nature 369, 568-571.

Keil, V.C., Funke, F., Zeug, A., Schild, D., and Muller, M. (2011). Ratiometric highresolution imaging of $\mathrm{JC}-1$ fluorescence reveals the subcellular heterogeneity of astrocytic mitochondria. Pflugers Archiv : European journal of physiology 462, 693-708.

Keller, G. (2005). Embryonic stem cell differentiation: emergence of a new era in biology and medicine. Genes Dev 19, 1129-1155. 
Kerner, J., Lee, K., Tandler, B., and Hoppel, C.L. (2012). VDAC proteomics: Posttranslation modifications. Biochimica et biophysica acta 1818, 1520-1525.

Kim, H.S., Patel, K., Muldoon-Jacobs, K., Bisht, K.S., Aykin-Burns, N., Pennington, J.D., van der Meer, R., Nguyen, P., Savage, J., Owens, K.M., et al. (2010). SIRT3 is a mitochondria-localized tumor suppressor required for maintenance of mitochondrial integrity and metabolism during stress. Cancer Cell $17,41-52$.

Kleinle, S., Wiesmann, U., Superti-Furga, A., Krahenbuhl, S., Boltshauser, E., Reichen, J., and Liechti-Gallati, S. (1997). Detection and characterization of mitochondrial DNA rearrangements in Pearson and Kearns-Sayre syndromes by long PCR. Hum Genet 100, 643-650.

Knight, S.W., Vulliamy, T.J., Heiss, N.S., Matthijs, G., Devriendt, K., Connor, J.M., D'Urso, M., Poustka, A., Mason, P.J., and Dokal, I. (1998). 1.4 Mb candidate gene region for $\mathrm{X}$ linked dyskeratosis congenita defined by combined haplotype and X chromosome inactivation analysis. J Med Genet 35, 993-996.

Koren, I., Raviv, Z., and Shoshan-Barmatz, V. (2010). Downregulation of voltagedependent anion channel-1 expression by RNA interference prevents cancer cell growth in vivo. Cancer biology \& therapy 9, 1046-1052.

Krishan, A. (1975). Rapid flow cytofluorometric analysis of mammalian cell cycle by propidium iodide staining. The Journal of cell biology $66,188-193$. 
Kubbutat, M.H., Jones, S.N., and Vousden, K.H. (1997). Regulation of p53 stability by Mdm2. Nature 387, 299-303.

Kuramitsu, M., Hamaguchi, I., Takuo, M., Masumi, A., Momose, H., Takizawa, K., Mochizuki, M., Naito, S., and Yamaguchi, K. (2008). Deficient RPS19 protein production induces cell cycle arrest in erythroid progenitor cells. $\mathrm{Br} \mathrm{J}$ Haematol 140, 348-359.

Lam, Y.W., Lamond, A.I., Mann, M., and Andersen, J.S. (2007). Analysis of nucleolar protein dynamics reveals the nuclear degradation of ribosomal proteins. Curr Biol 17, 749-760.

Lebel, C.P., and Bondy, S.C. (1990). Sensitive and rapid quantitation of oxygen reactive species formation in rat synaptosomes. Neurochemistry international 17 , 435-440.

Lee, H.F., Lee, H.J., Chi, C.S., Tsai, C.R., Chang, T.K., and Wang, C.J. (2007). The neurological evolution of Pearson syndrome: case report and literature review. European journal of paediatric neurology : EJPN : official journal of the European Paediatric Neurology Society 11, 208-214.

Legrand, O., Perrot, J.Y., Simonin, G., Baudard, M., and Marie, J.P. (2001). JC-1: a very sensitive fluorescent probe to test Pgp activity in adult acute myeloid leukemia. Blood 97, 502-508. 
Leung, R., Cuddy, K., Wang, Y., Rommens, J., and Glogauer, M. (2011). Sbds is required for Rac2-mediated monocyte migration and signaling downstream of RANK during osteoclastogenesis. Blood 117, 2044-2053.

Lindstrom, M.S., Deisenroth, C., and Zhang, Y. (2007). Putting a finger on growth surveillance: insight into MDM2 zinc finger-ribosomal protein interactions. Cell Cycle 6, 434-437.

Lipton, J.M., and Ellis, S.R. (2009). Diamond-Blackfan anemia: diagnosis, treatment, and molecular pathogenesis. Hematology/oncology clinics of North America 23, 261-282.

Lipton, J.M., and Ellis, S.R. (2010). Diamond Blackfan anemia 2008-2009: broadening the scope of ribosome biogenesis disorders. Curr Opin Pediatr 22, 12-19.

Lipton, J.M., Kudisch, M., Gross, R., and Nathan, D.G. (1986). Defective erythroid progenitor differentiation system in congenital hypoplastic (DiamondBlackfan) anemia. Blood 67, 962-968.

Liu, J.M., and Ellis, S.R. (2006). Ribosomes and marrow failure: coincidental association or molecular paradigm? Blood 107, 4583-4588. 
Lohrum, M.A., Ashcroft, M., Kubbutat, M.H., and Vousden, K.H. (2000).

Contribution of two independent MDM2-binding domains in p14(ARF) to p53 stabilization. Curr Biol 10, 539-542.

Lohrum, M.A., Ludwig, R.L., Kubbutat, M.H., Hanlon, M., and Vousden, K.H. (2003). Regulation of HDM2 activity by the ribosomal protein L11. Cancer Cell 3, $577-587$.

Lugli, E., Troiano, L., and Cossarizza, A. (2007). Polychromatic analysis of mitochondrial membrane potential using JC-1. Current protocols in cytometry / editorial board, J. Paul Robinson, managing editor ... [et al.] Chapter 7, Unit7 32.

Magnuson, B., Ekim, B., and Fingar, D.C. (2012). Regulation and function of ribosomal protein S6 kinase (S6K) within mTOR signalling networks. Biochem J $441,1-21$

Makitie, O. (1992). Cartilage-hair hypoplasia in Finland: epidemiological and genetic aspects of 107 patients. J Med Genet 29, 652-655.

Mantzourani, M., Konstantopoulos, K., Voskaridou, E., and Loukopoulos, D. (1995). Molecular genetics of "X-linked" sideroblastic anemia. American journal of hematology 48, 131.

Maserati, E., Pressato, B., Valli, R., Minelli, A., Sainati, L., Patitucci, F., Marletta, C., Mastronuzzi, A., Poli, F., Lo Curto, F., et al. (2009). The route to development 
of myelodysplastic syndrome/acute myeloid leukaemia in Shwachman-Diamond syndrome: the role of ageing, karyotype instability, and acquired chromosome anomalies. Br J Haematol 145, 190-197.

Matsson, H., Davey, E.J., Draptchinskaia, N., Hamaguchi, I., Ooka, A., Leveen, P., Forsberg, E., Karlsson, S., and Dahl, N. (2004). Targeted disruption of the ribosomal protein S19 gene is lethal prior to implantation. Mol Cell Biol 24, $4032-4037$.

McDonald, B.M., Wydro, M.M., Lightowlers, R.N., and Lakey, J.H. (2009). Probing the orientation of yeast VDAC1 in vivo. FEBS Lett 583, 739-742.

McGowan, K.A., Li, J.Z., Park, C.Y., Beaudry, V., Tabor, H.K., Sabnis, A.J., Zhang, W., Fuchs, H., de Angelis, M.H., Myers, R.M., et al. (2008). Ribosomal mutations cause p53-mediated dark skin and pleiotropic effects. Nat Genet 40, 963-970.

McGowan, K.A., Pang, W.W., Bhardwaj, R., Perez, M.G., Pluvinage, J.V., Glader, B.E., Malek, R., Mendrysa, S.M., Weissman, I.L., Park, C.Y., et al. (2011). Reduced ribosomal protein gene dosage and p53 activation in low-risk myelodysplastic syndrome. Blood 118, 3622-3633.

McKusick, V.A., Eldridge, R., Hostetler, J.A., Ruangwit, U., and Egeland, J.A. (1965). Dwarfism in the Amish. li. Cartilage-Hair Hypoplasia. Bulletin of the Johns Hopkins Hospital 116, 285-326. 
McShane, M.A., Hammans, S.R., Sweeney, M., Holt, I.J., Beattie, T.J., Brett, E.M., and Harding, A.E. (1991). Pearson syndrome and mitochondrial encephalomyopathy in a patient with a deletion of mtDNA. Am J Hum Genet 48, $39-42$.

Meetei, A.R., Sechi, S., Wallisch, M., Yang, D., Young, M.K., Joenje, H., Hoatlin, M.E., and Wang, W. (2003). A multiprotein nuclear complex connects Fanconi anemia and Bloom syndrome. Mol Cell Biol 23, 3417-3426.

Menne, T.F., Goyenechea, B., Sanchez-Puig, N., Wong, C.C., Tonkin, L.M., Ancliff, P.J., Brost, R.L., Costanzo, M., Boone, C., and Warren, A.J. (2007). The Shwachman-Bodian-Diamond syndrome protein mediates translational activation of ribosomes in yeast. Nat Genet 39, 486-495.

Messina, A., Reina, S., Guarino, F., and De Pinto, V. (2012). VDAC isoforms in mammals. Biochimica et biophysica acta 1818, 1466-1476.

Metcalf, D. (2007). On hematopoietic stem cell fate. Immunity 26, 669-673.

Miller, O.L., Jr., and Beatty, B.R. (1969). Visualization of nucleolar genes. Science $164,955-957$.

Miluzio, A., Beugnet, A., Volta, V., and Biffo, S. (2009). Eukaryotic initiation factor 6 mediates a continuum between 605 ribosome biogenesis and translation. EMBO reports 10, 459-465. 
Miyake, K., Flygare, J., Kiefer, T., Utsugisawa, T., Richter, J., Ma, Z., Wiznerowicz, M., Trono, D., and Karlsson, S. (2005). Development of cellular models for ribosomal protein S19 (RPS19)-deficient diamond-blackfan anemia using inducible expression of siRNA against RPS19. Molecular therapy : the journal of the American Society of Gene Therapy 11, 627-637.

Miyake, K., Utsugisawa, T., Flygare, J., Kiefer, T., Hamaguchi, I., Richter, J., and Karlsson, S. (2008). Ribosomal protein S19 deficiency leads to reduced proliferation and increased apoptosis but does not affect terminal erythroid differentiation in a cell line model of Diamond-Blackfan anemia. Stem Cells 26, 323-329.

Moldovan, G.L., and D'Andrea, A.D. (2009). How the fanconi anemia pathway guards the genome. Annu Rev Genet 43, 223-249.

Montanaro, L., Mazzini, G., Barbieri, S., Vici, M., Nardi-Pantoli, A., Govoni, M., Donati, G., Trere, D., and Derenzini, M. (2007). Different effects of ribosome biogenesis inhibition on cell proliferation in retinoblastoma protein- and p53deficient and proficient human osteosarcoma cell lines. Cell Prolif 40, 532-549.

Moore, J.B.t., Farrar, J.E., Arceci, R.J., Liu, J.M., and Ellis, S.R. (2010). Distinct ribosome maturation defects in yeast models of Diamond-Blackfan anemia and Shwachman-Diamond syndrome. Haematologica 95, 57-64. 
Moy, T.I., and Silver, P.A. (1999). Nuclear export of the small ribosomal subunit requires the ran-GTPase cycle and certain nucleoporins. Genes Dev 13, 2118-2133.

Moy, T.I., and Silver, P.A. (2002). Requirements for the nuclear export of the small ribosomal subunit. J Cell Sci 115, 2985-2995.

Nakanishi, K., Taniguchi, T., Ranganathan, V., New, H.V., Moreau, L.A., Stotsky, M., Mathew, C.G., Kastan, M.B., Weaver, D.T., and D'Andrea, A.D. (2002). Interaction of FANCD2 and NBS1 in the DNA damage response. Nat Cell Biol 4, 913-920.

Narla, A., and Ebert, B.L. (2010). Ribosomopathies: human disorders of ribosome dysfunction. Blood 115, 3196-3205.

Nathan, D.G., Clarke, B.J., Hillman, D.G., Alter, B.P., and Housman, D.E. (1978). Erythroid precursors in congenital hypoplastic (Diamond-Blackfan) anemia. J Clin Invest 61, 489-498.

Ng, C.L., Waterman, D.G., Koonin, E.V., Walters, A.D., Chong, J.P., Isupov, M.N., Lebedev, A.A., Bunka, D.H., Stockley, P.G., Ortiz-Lombardia, M., et al. (2009). Conformational flexibility and molecular interactions of an archaeal homologue of the Shwachman-Bodian-Diamond syndrome protein. BMC structural biology 9 , 32. 
Niaudet, P., Heidet, L., Munnich, A., Schmitz, J., Bouissou, F., Gubler, M.C., and Rotig, A. (1994). Deletion of the mitochondrial DNA in a case of de Toni-DebreFanconi syndrome and Pearson syndrome. Pediatr Nephrol 8, 164-168.

Nihrane, A., Sezgin, G., Dsilva, S., Dellorusso, P., Yamamoto, K., Ellis, S.R., and Liu, J.M. (2009). Depletion of the Shwachman-Diamond syndrome gene product, SBDS, leads to growth inhibition and increased expression of OPG and VEGF-A. Blood Cells Mol Dis 42, 85-91.

Ofir-Rosenfeld, Y., Boggs, K., Michael, D., Kastan, M.B., and Oren, M. (2008). Mdm2 regulates p53 mRNA translation through inhibitory interactions with ribosomal protein L26. Mol Cell 32, 180-189.

Ohene-Abuakwa, Y., Orfali, K.A., Marius, C., and Ball, S.E. (2005). Two-phase culture in Diamond Blackfan anemia: localization of erythroid defect. Blood 105, 838-846.

Orelio, C., and Kuijpers, T.W. (2009). Shwachman-Diamond syndrome neutrophils have altered chemoattractant-induced F-actin polymerization and polarization characteristics. Haematologica 94, 409-413.

Orelio, C., van der Sluis, R.M., Verkuijlen, P., Nethe, M., Hordijk, P.L., van den Berg, T.K., and Kuijpers, T.W. (2011). Altered intracellular localization and mobility of SBDS protein upon mutation in Shwachman-Diamond syndrome. PloS one 6, e20727. 
Orelio, C., Verkuijlen, P., Geissler, J., van den Berg, T.K., and Kuijpers, T.W. (2009). SBDS expression and localization at the mitotic spindle in human myeloid progenitors. PloS one 4, e7084.

Oyama, Y., Hayashi, A., Ueha, T., and Maekawa, K. (1994). Characterization of 2',7'-dichlorofluorescin fluorescence in dissociated mammalian brain neurons: estimation on intracellular content of hydrogen peroxide. Brain Res $635,113-117$.

Pagon, R.A., and Bird, T.D. (1993). X-Linked Sideroblastic Anemia and Ataxia. In GeneReviews, R.A. Pagon, T.D. Bird, C.R. Dolan, K. Stephens, and M.P. Adam, eds. (Seattle (WA)).

Panse, V.G. (2011). Getting ready to translate: cytoplasmic maturation of eukaryotic ribosomes. Chimia 65, 765-769.

Panse, V.G., and Johnson, A.W. (2010). Maturation of eukaryotic ribosomes: acquisition of functionality. Trends in biochemical sciences 35, 260-266.

Parikh, S., and Bessler, M. (2012). Recent insights into inherited bone marrow failure syndromes. Curr Opin Pediatr 24, 23-32.

Pearson, H.A., Lobel, J.S., Kocoshis, S.A., Naiman, J.L., Windmiller, J., Lammi, A.T., Hoffman, R., and Marsh, J.C. (1979). A new syndrome of refractory sideroblastic anemia with vacuolization of marrow precursors and exocrine pancreatic dysfunction. J Pediatr 95, 976-984. 
Perdahl, E.B., Naprstek, B.L., Wallace, W.C., and Lipton, J.M. (1994). Erythroid failure in Diamond-Blackfan anemia is characterized by apoptosis. Blood 83, $645-650$.

Pichierri, P., Franchitto, A., and Rosselli, F. (2004). BLM and the FANC proteins collaborate in a common pathway in response to stalled replication forks. EMBO J 23, 3154-3163.

Posnick, J.C., and Ruiz, R.L. (2000). Treacher Collins syndrome: current evaluation, treatment, and future directions. The Cleft palate-craniofacial journal : official publication of the American Cleft Palate-Craniofacial Association 37, 434.

Raaijmakers, M.H., Mukherjee, S., Guo, S., Zhang, S., Kobayashi, T., Schoonmaker, J.A., Ebert, B.L., Al-Shahrour, F., Hasserjian, R.P., Scadden, E.O., et al. (2010). Bone progenitor dysfunction induces myelodysplasia and secondary leukaemia. Nature 464, 852-857.

Raskind, W.H., Wijsman, E., Pagon, R.A., Cox, T.C., Bawden, M.J., May, B.K., and Bird, T.D. (1991). X-linked sideroblastic anemia and ataxia: linkage to phosphoglycerate kinase at Xq13. Am J Hum Genet 48, 335-341.

Rawls, A.S., Gregory, A.D., Woloszynek, J.R., Liu, F., and Link, D.C. (2007). Lentiviral-mediated RNAi inhibition of Sbds in murine hematopoietic progenitors impairs their hematopoietic potential. Blood 110, 2414-2422. 
Reers, M., Smiley, S.T., Mottola-Hartshorn, C., Chen, A., Lin, M., and Chen, L.B. (1995). Mitochondrial membrane potential monitored by JC-1 dye. Methods Enzymol 260, 406-417.

Ribbeck, K., and Gorlich, D. (2002). The permeability barrier of nuclear pore complexes appears to operate via hydrophobic exclusion. EMBO J 21, 2664-2671.

Ridanpaa, M., Sistonen, P., Rockas, S., Rimoin, D.L., Makitie, O., and Kaitila, I. (2002). Worldwide mutation spectrum in cartilage-hair hypoplasia: ancient founder origin of the major70A--> G mutation of the untranslated RMRP. Eur $\mathrm{J}$ Hum Genet 10, 439-447.

Robledo, S., Idol, R.A., Crimmins, D.L., Ladenson, J.H., Mason, P.J., and Bessler, M. (2008). The role of human ribosomal proteins in the maturation of rRNA and ribosome production. RNA 14, 1918-1929.

Rosenberg, P.S., Huang, Y., and Alter, B.P. (2004). Individualized risks of first adverse events in patients with Fanconi anemia. Blood 104, 350-355.

Rosenberg, P.S., Socie, G., Alter, B.P., and Gluckman, E. (2005). Risk of head and neck squamous cell cancer and death in patients with Fanconi anemia who did and did not receive transplants. Blood 105, 67-73. 
Rosenkranz, A.R., Schmaldienst, S., Stuhlmeier, K.M., Chen, W., Knapp, W., and Zlabinger, G.J. (1992). A microplate assay for the detection of oxidative products using 2',7'-dichlorofluorescin-diacetate. Journal of immunological methods 156 , $39-45$.

Rothbaum, R., Perrault, J., Vlachos, A., Cipolli, M., Alter, B.P., Burroughs, S., Durie, P., Elghetany, M.T., Grand, R., Hubbard, V., et al. (2002). ShwachmanDiamond syndrome: report from an international conference. J Pediatr 141, 266-270.

Rotig, A., Cormier, V., Blanche, S., Bonnefont, J.P., Ledeist, F., Romero, N., Schmitz, J., Rustin, P., Fischer, A., Saudubray, J.M., et al. (1990). Pearson's marrow-pancreas syndrome. A multisystem mitochondrial disorder in infancy. $\mathrm{J}$ Clin Invest 86, 1601-1608.

Rouquette, J., Choesmel, V., and Gleizes, P.E. (2005). Nuclear export and cytoplasmic processing of precursors to the $40 \mathrm{~S}$ ribosomal subunits in mammalian cells. EMBO J 24, 2862-2872.

Rujkijyanont, P., Adams, S.L., Beyene, J., and Dror, Y. (2009). Bone marrow cells from patients with Shwachman-Diamond syndrome abnormally express genes involved in ribosome biogenesis and RNA processing. $\mathrm{Br} \mathrm{J}$ Haematol 145, 806-815. 
Rujkijyanont, P., Watanabe, K., Ambekar, C., Wang, H., Schimmer, A., Beyene, J., and Dror, Y. (2008). SBDS-deficient cells undergo accelerated apoptosis through the Fas-pathway. Haematologica 93, 363-371.

S, B. (1997). From Genes to Cells (Wiley-Liss).

Salvioli, S., Ardizzoni, A., Franceschi, C., and Cossarizza, A. (1997). JC-1, but not DiOC6(3) or rhodamine 123, is a reliable fluorescent probe to assess delta psi changes in intact cells: implications for studies on mitochondrial functionality during apoptosis. FEBS Lett 411, 77-82.

Santorelli, F.M., Barmada, M.A., Pons, R., Zhang, L.L., and DiMauro, S. (1996). Leigh-type neuropathology in Pearson syndrome associated with impaired ATP production and a novel mtDNA deletion. Neurology 47, 1320-1323.

Savage, S.A., and Alter, B.P. (2008). The role of telomere biology in bone marrow failure and other disorders. Mechanisms of ageing and development 129, 35-47.

Savage, S.A., and Alter, B.P. (2009). Dyskeratosis congenita. Hematology/ oncology clinics of North America 23, 215-231.

Savage, S.A., Dokal, I., Armanios, M., Aubert, G., Cowen, E.W., Domingo, D.L., Giri, N., Greene, M.H., Orchard, P.J., Tolar, J., et al. (2009). Dyskeratosis congenita: the first NIH clinical research workshop. Pediatr Blood Cancer 53, 520-523. 
Schumacker, P.T. (2006). Reactive oxygen species in cancer cells: live by the sword, die by the sword. Cancer Cell 10, 175-176.

Seiser, R.M., Sundberg, A.E., Wollam, B.J., Zobel-Thropp, P., Baldwin, K., Spector, M.D., and Lycan, D.E. (2006). Ltv1 is required for efficient nuclear export of the ribosomal small subunit in Saccharomyces cerevisiae. Genetics $174,679-691$.

Sen, S., Wang, H., Nghiem, C.L., Zhou, K., Yau, J., Tailor, C.S., Irwin, M.S., and Dror, Y. (2011). The ribosome-related protein, SBDS, is critical for normal erythropoiesis. Blood 118, 6407-6417.

Senger, B., Lafontaine, D.L., Graindorge, J.S., Gadal, O., Camasses, A., Sanni, A., Garnier, J.M., Breitenbach, M., Hurt, E., and Fasiolo, F. (2001). The nucle(ol)ar Tif6p and Efl1p are required for a late cytoplasmic step of ribosome synthesis. Mol Cell 8, 1363-1373.

Shammas, C., Menne, T.F., Hilcenko, C., Michell, S.R., Goyenechea, B., Boocock, G.R., Durie, P.R., Rommens, J.M., and Warren, A.J. (2005). Structural and mutational analysis of the SBDS protein family. Insight into the leukemiaassociated Shwachman-Diamond Syndrome. J Biol Chem 280, 19221-19229.

Shapiro, H. (2003). Practical Flow Cytometry, 4th edition edn (Wiley \& Sons ). 
Shimamura, A., and Alter, B.P. (2010). Pathophysiology and management of inherited bone marrow failure syndromes. Blood reviews 24, 101-122.

Shoshan-Barmatz, V., De Pinto, V., Zweckstetter, M., Raviv, Z., Keinan, N., and Arbel, N. (2010a). VDAC, a multi-functional mitochondrial protein regulating cell life and death. Molecular aspects of medicine 31, 227-285.

Shoshan-Barmatz, V., Keinan, N., Abu-Hamad, S., Tyomkin, D., and Aram, L. (2010b). Apoptosis is regulated by the VDAC1 N-terminal region and by VDAC oligomerization: release of cytochrome c, AIF and Smac/Diablo. Biochimica et biophysica acta 1797, 1281-1291.

Shwachman, H., Diamond, L.K., Oski, F.A., and Khaw, K.T. (1964). The Syndrome of Pancreatic Insufficiency and Bone Marrow Dysfunction. J Pediatr $65,645-663$.

Simeonova, E., Garstka, M., Koziol-Lipinska, J., and Mostowska, A. (2004). Monitoring the mitochondrial transmembrane potential with the JC-1 fluorochrome in programmed cell death during mesophyll leaf senescence. Protoplasma 223, 143-153.

Singh, T.R., Bakker, S.T., Agarwal, S., Jansen, M., Grassman, E., Godthelp, B.C., Ali, A.M., Du, C.H., Rooimans, M.A., Fan, Q., et al. (2009). Impaired FANCD2 monoubiquitination and hypersensitivity to camptothecin uniquely characterize Fanconi anemia complementation group M. Blood 114, 174-180. 
Skokowa, J., Fobiwe, J.P., Dan, L., Thakur, B.K., and Welte, K. (2009). Neutrophil elastase is severely down-regulated in severe congenital neutropenia independent of ELA2 or HAX1 mutations but dependent on LEF-1. Blood 114, 3044-3051.

Smiley, S.T., Reers, M., Mottola-Hartshorn, C., Lin, M., Chen, A., Smith, T.W., Steele, G.D., Jr., and Chen, L.B. (1991). Intracellular heterogeneity in mitochondrial membrane potentials revealed by a J-aggregate-forming lipophilic cation JC-1. Proc Natl Acad Sci U S A 88, 3671-3675.

Smith, O.P., Hann, I.M., Chessells, J.M., Reeves, B.R., and Milla, P. (1996). Haematological abnormalities in Shwachman-Diamond syndrome. $\mathrm{Br} \mathrm{J}$ Haematol 94, 279-284.

Sonenberg, N., and Hinnebusch, A.G. (2009). Regulation of translation initiation in eukaryotes: mechanisms and biological targets. Cell 136, 731-745.

Soulier, J. (2011). Fanconi anemia. Hematology Am Soc Hematol Educ Program $2011,492-497$.

Stage-Zimmermann, T., Schmidt, U., and Silver, P.A. (2000). Factors affecting nuclear export of the $60 \mathrm{~S}$ ribosomal subunit in vivo. Molecular biology of the cell 11, 3777-3789. 
Sun, X.X., Dai, M.S., and Lu, H. (2007). 5-fluorouracil activation of p53 involves an MDM2-ribosomal protein interaction. J Biol Chem 282, 8052-8059.

Sutherland, G.R., Baker, E., Callen, D.F., Hyland, V.J., May, B.K., Bawden, M.J., Healy, H.M., and Borthwick, I.A. (1988). 5-Aminolevulinate synthase is at 3p21 and thus not the primary defect in X-linked sideroblastic anemia. Am J Hum Genet 43, 331-335.

Tamary, H., and Alter, B.P. (2007). Current diagnosis of inherited bone marrow failure syndromes. Pediatric hematology and oncology 24, 87-99.

Tamary, H., Nishri, D., Yacobovich, J., Zilber, R., Dgany, O., Krasnov, T., Aviner, S., Stepensky, P., Ravel-Vilk, S., Bitan, M., et al. (2010). Frequency and natural history of inherited bone marrow failure syndromes: the Israeli Inherited Bone Marrow Failure Registry. Haematologica 95, 1300-1307.

Taniguchi, T., Garcia-Higuera, I., Xu, B., Andreassen, P.R., Gregory, R.C., Kim, S.T., Lane, W.S., Kastan, M.B., and D'Andrea, A.D. (2002). Convergence of the fanconi anemia and ataxia telangiectasia signaling pathways. Cell 109, 459-472.

Theil, C., Briese, V., Gerber, B., and Richter, D.U. (2011). The effects of different lignans and isoflavones, tested as aglycones and glycosides, on hormone receptor-positive and -negative breast carcinoma cells in vitro. Archives of gynecology and obstetrics 284, 459-465. 
Thomas, F., and Kutay, U. (2003). Biogenesis and nuclear export of ribosomal subunits in higher eukaryotes depend on the CRM1 export pathway. J Cell Sci 116, 2409-2419.

Thompson, A.A., Woodruff, K., Feig, S.A., Nguyen, L.T., and Schanen, N.C. (2001). Congenital thrombocytopenia and radio-ulnar synostosis: a new familial syndrome. Br J Haematol 113, 866-870.

Topaloglu, R., Lebre, A.S., Demirkaya, E., Kuskonmaz, B., Coskun, T., Orhan, D., Gurgey, A., and Gumruk, F. (2008). Two new cases with Pearson syndrome and review of Hacettepe experience. The Turkish journal of pediatrics 50, 572-576.

Trayner, I.D., Rayner, A.P., Freeman, G.E., and Farzaneh, F. (1995). Quantitative multiwell myeloid differentiation assay using dichlorodihydrofluorescein diacetate (H2DCF-DA) or dihydrorhodamine 123 (H2R123). Journal of immunological methods $186,275-284$.

Tsai, P.H., Arkin, S., and Lipton, J.M. (1989). An intrinsic progenitor defect in Diamond-Blackfan anaemia. Br J Haematol 73, 112-120.

Tsangaris, E., Klaassen, R., Fernandez, C.V., Yanofsky, R., Shereck, E., Champagne, J., Silva, M., Lipton, J.H., Brossard, J., Michon, B., et al. (2011). Genetic analysis of inherited bone marrow failure syndromes from one prospective, comprehensive and population-based cohort and identification of novel mutations. J Med Genet 48, 618-628. 
Uechi, T., Nakajima, Y., Chakraborty, A., Torihara, H., Higa, S., and Kenmochi, N. (2008). Deficiency of ribosomal protein S19 during early embryogenesis leads to reduction of erythrocytes in a zebrafish model of Diamond-Blackfan anemia. Hum Mol Genet 17, 3204-3211.

Valdez, B.C., Henning, D., So, R.B., Dixon, J., and Dixon, M.J. (2004). The Treacher Collins syndrome (TCOF1) gene product is involved in ribosomal DNA gene transcription by interacting with upstream binding factor. Proc Natl Acad Sci U S A 101, 10709-10714.

Valenzuela, D.M., Chaudhuri, A., and Maitra, U. (1982). Eukaryotic ribosomal subunit anti-association activity of calf liver is contained in a single polypeptide chain protein of $\mathrm{Mr}=25,500$ (eukaryotic initiation factor 6). J Biol Chem 257, 7712-7719.

van den Ouweland, J.M., de Klerk, J.B., van de Corput, M.P., Dirks, R.W., Raap, A.K., Scholte, H.R., Huijmans, J.G., Hart, L.M., Bruining, G.J., and Maassen, J.A. (2000). Characterization of a novel mitochondrial DNA deletion in a patient with a variant of the Pearson marrow-pancreas syndrome. Eur J Hum Genet 8, 195-203.

Vitiello, S.P., Benedict, J.W., Padilla-Lopez, S., and Pearce, D.A. (2010). Interaction between Sdo1p and Btn1p in the Saccharomyces cerevisiae model for Batten disease. Hum Mol Genet 19, 931-942. 
Vlachos, A., Ball, S., Dahl, N., Alter, B.P., Sheth, S., Ramenghi, U., Meerpohl, J., Karlsson, S., Liu, J.M., Leblanc, T., et al. (2008). Diagnosing and treating Diamond Blackfan anaemia: results of an international clinical consensus conference. Br J Haematol 142, 859-876.

Wang, W. (2007). Emergence of a DNA-damage response network consisting of Fanconi anaemia and BRCA proteins. Nature reviews. Genetics 8, 735-748.

Wang, X., Kennedy, R.D., Ray, K., Stuckert, P., Ellenberger, T., and D'Andrea, A.D. (2007). Chk1-mediated phosphorylation of FANCE is required for the Fanconi anemia/BRCA pathway. Mol Cell Biol 27, 3098-3108.

Warner, J.R. (1990). The nucleolus and ribosome formation. Current opinion in cell biology 2, 521-527.

Warner, J.R., and McIntosh, K.B. (2009). How common are extraribosomal functions of ribosomal proteins? Mol Cell 34, 3-11.

Watanabe, K., Ambekar, C., Wang, H., Ciccolini, A., Schimmer, A.D., and Dror, Y. (2009). SBDS-deficiency results in specific hypersensitivity to Fas stimulation and accumulation of Fas at the plasma membrane. Apoptosis : an international journal on programmed cell death $14,77-89$.

Watson, J.V., Chambers, S.H., and Smith, P.J. (1987). A pragmatic approach to the analysis of DNA histograms with a definable G1 peak. Cytometry 8, 1-8. 
Webb, A. (2002). Statistical Pattern Recognition (New York John Wiley \& Sons Inc ), pp. 41-49.

Welte, K., and Zeidler, C. (2009). Severe congenital neutropenia. Hematology/ oncology clinics of North America 23, 307-320.

Wessels, D., Srikantha, T., Yi, S., Kuhl, S., Aravind, L., and Soll, D.R. (2006). The Shwachman-Bodian-Diamond syndrome gene encodes an RNA-binding protein that localizes to the pseudopod of Dictyostelium amoebae during chemotaxis. J Cell Sci 119, 370-379.

Williams, T.B., Daniels, M., Puthenveetil, G., Chang, R., Wang, R.Y., and Abdenur, J.E. (2012). Pearson syndrome: Unique endocrine manifestations including Neonatal Diabetes and adrenal insufficiency. Molecular genetics and metabolism 106, 104-107.

Wong, C.C., Traynor, D., Basse, N., Kay, R.R., and Warren, A.J. (2011). Defective ribosome assembly in Shwachman-Diamond syndrome. Blood 118, 4305-4312.

Wurm, C.A., Neumann, D., Lauterbach, M.A., Harke, B., Egner, A., Hell, S.W., and Jakobs, S. (2011). Nanoscale distribution of mitochondrial import receptor Tom20 is adjusted to cellular conditions and exhibits an inner-cellular gradient. Proc Natl Acad Sci U S A 108, 13546-13551. 
Xia, J., and Link, D.C. (2008). Severe congenital neutropenia and the unfolded protein response. Current opinion in hematology 15, 1-7.

Yadavilli, S., Mayo, L.D., Higgins, M., Lain, S., Hegde, V., and Deutsch, W.A. (2009). Ribosomal protein S3: A multi-functional protein that interacts with both p53 and MDM2 through its KH domain. DNA Repair (Amst) 8, 1215-1224.

Yamaguchi, M., Fujimura, K., Kanegane, H., Toga-Yamaguchi, H., Chopra, R., and Okamura, N. (2011). Mislocalization or low expression of mutated Shwachman-Bodian-Diamond syndrome protein. International journal of hematology 94, 54-62.

Yamaguchi, M., Fujimura, K., Toga, H., Khwaja, A., Okamura, N., and Chopra, R. (2007). Shwachman-Diamond syndrome is not necessary for the terminal maturation of neutrophils but is important for maintaining viability of granulocyte precursors. Exp Hematol 35, 579-586.

Zeidler, C., Germeshausen, M., Klein, C., and Welte, K. (2009). Clinical implications of ELA2-, HAX1-, and G-CSF-receptor (CSF3R) mutations in severe congenital neutropenia. Br J Haematol 144, 459-467.

Zemp, I., and Kutay, U. (2007). Nuclear export and cytoplasmic maturation of ribosomal subunits. FEBS Lett 581, 2783-2793. 
Zemp, I., Wild, T., O'Donohue, M.F., Wandrey, F., Widmann, B., Gleizes, P.E., and Kutay, U. (2009). Distinct cytoplasmic maturation steps of 40 S ribosomal subunit precursors require hRio2. The Journal of cell biology $185,1167-1180$.

Zhang, J., Harnpicharnchai, P., Jakovljevic, J., Tang, L., Guo, Y., Oeffinger, M., Rout, M.P., Hiley, S.L., Hughes, T., and Woolford, J.L., Jr. (2007). Assembly factors Rpf2 and Rrs1 recruit 5S rRNA and ribosomal proteins rpL5 and rpL11 into nascent ribosomes. Genes Dev 21, 2580-2592.

Zhang, S., Shi, M., Hui, C.C., and Rommens, J.M. (2006). Loss of the mouse ortholog of the shwachman-diamond syndrome gene (Sbds) results in early embryonic lethality. Mol Cell Biol 26, 6656-6663.

Zhang, Y., Wolf, G.W., Bhat, K., Jin, A., Allio, T., Burkhart, W.A., and Xiong, Y. (2003). Ribosomal protein L11 negatively regulates oncoprotein MDM2 and mediates a p53-dependent ribosomal-stress checkpoint pathway. Mol Cell Biol 23, 8902-8912.

Zhu, Y., Poyurovsky, M.V., Li, Y., Biderman, L., Stahl, J., Jacq, X., and Prives, C. (2009). Ribosomal protein S7 is both a regulator and a substrate of MDM2. Mol Cell 35, 316-326. 


\section{APPENDIX I}

\section{$\underline{\text { Initial Remarks }}$}

Research studies that did not easily fall under the same umbrella as the major works of my dissertation are found in the following sections. These topics include verification of ribosome defects in an inducible mouse model of Rps19 shRNA and mouse embryonic stem cell (mES) models, all relating to Diamond Blackfan anemia (DBA). The mES models mimic haploinsufficiency for both Rps 19 and $R p / 5$ in different cell lines, providing a tool for comparison of different DBA genes. 


\title{
Supplemental Section 1: Mouse Embryonic Stem Cell Model
}

\author{
$\underline{\text { Introduction }}$
}

Macrocytic anemia of variable severity is the unifying feature of DBA diagnosis (Lipton et al., 1986). Bone marrow biopsy is central in classifying this inherited bone marrow failure syndrome. Classical DBA shows a red cell specific propensity for apoptosis in erythroid progenitors with an otherwise normocellular bone marrow (Miyake et al., 2008; Ohene-Abuakwa et al., 2005; Perdahl et al., 1994; Tsai et al., 1989). Having a valid animal model to gain understanding of the molecular mechanisms that result in the vast array of features associated with a mutlifactoral disease such as DBA is of paramount importance. In addition to potential advances in whole animal models, recapitulating central events in hematopoiesis in a tissue culture system could provide an alternative methodology to study DBA pathophysiology in the absence of a suitable animal model. Stem cell cultures have been used to mimic differentiation of progenitors and study erythropoiesis defects in other contexts, but not in DBA (Choi, 1998; Keller, 2005) To this end, mouse embryonic stem cells were used to mimic haploinsufficiency for known DBA genes. These gene trap constructs were created by retroviral insertion within the genes for $R p s 19$ or $R p / 5$ that causes a premature termination of the gene transcript in only one copy of the gene (Keller, 2005). With appropriate culture conditions and appropriate cytokine stimulation they can be induced to differentiation into blood lineage specific colonies in the manner shown in Fig. S1. 


\section{In vivo}

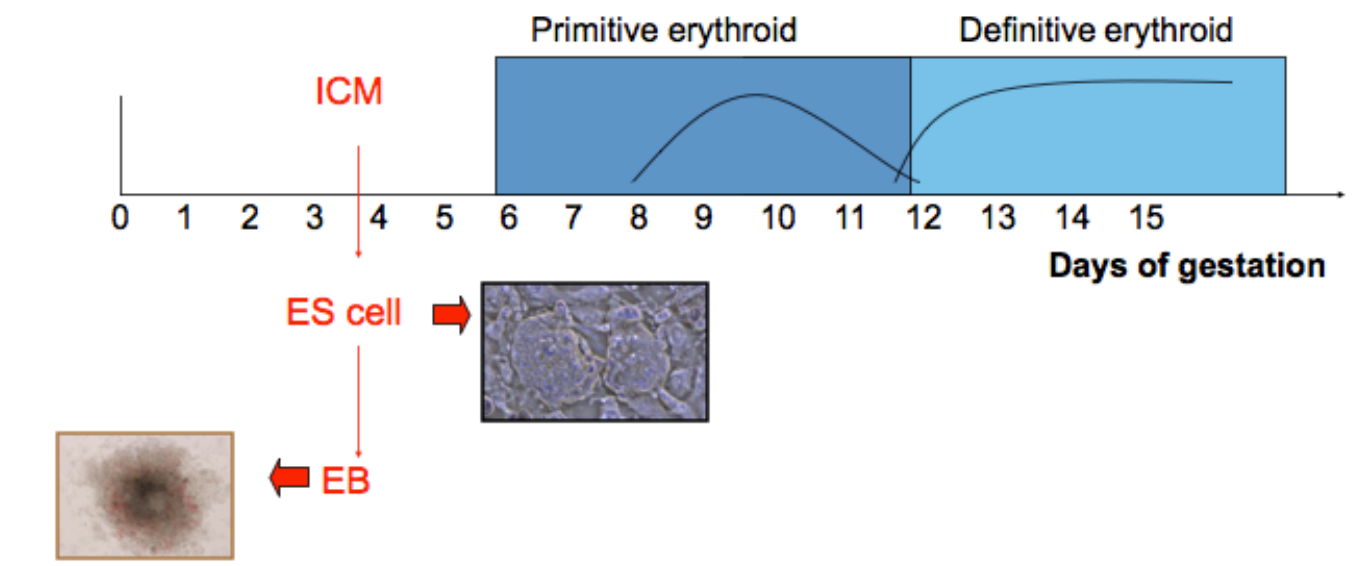

In vitro

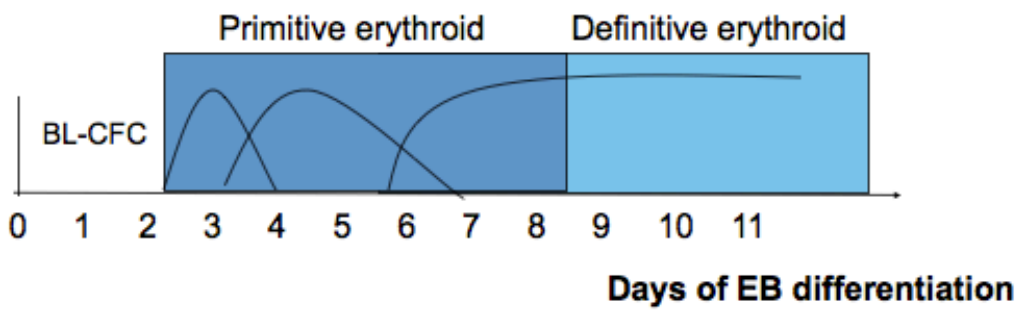

Fig S1: Embryonic stem cell culture recapitulates hematopoiesis in mouse models.

Differentiation of mES models can be used to mimic the in vivo erythrocyte development with representative images of ES cell and embryoid bodies (EBs) shown in the inset pictures. Adapted from previous works (Keller, 2005). 
Stem cells in this system can be grown in an undifferentiated state to represent the HSC multipotent progenitor or undergo primary differentiation in methylcellulose media to form embryoid bodies. The viscous nature of methylcellulose is necessary for the sensitive stem cell progenitors to receive appropriate structural interactions with the surrounding environment to differentiate. On specific days of differentiation, as outlined in the in vitro time line of Fig. S1, the EBs can be harvested and induced to form primitive or definitive erythroid colonies. Primitive erythroid colonies express variants of hemoglobin specific to fetal development, such as Hbb-bh1. This can be thought of as analogous to the fetal hemoglobin chains produced during human embryogenesis $(\mathrm{Hb}-\mathrm{F}=$ Hemoglobin Fetal). Definitive erythropoiesis is responsible for the production of red cells in adult animals and can be modeled by harvesting EBs later in their development before inducing differentiation with SCF (Stem cell factor), IL-3, IL-6, and epo to form burst forming units of the erythroid lineage (BFU-Es). In mice adult hemoglobin is Hbb-b1.

The mES models represented here were described earlier in the materials and methods section, but will be briefly reviewed here. Two different cell lines heterozygous for Rps19 were used in an attempt to recapitulate the pathology of haploinsufficiency for Rps19. The $\mathrm{S} 17-10 \mathrm{H} 1$ line was created by genetrap insertion between exon 2 and 3 in the Rps 19 gene of the Ak7 mouse parental line. A "rescue" of this cell line was generated by electroporation of a plasmid expressing Rps19 from a cDNA insert and termed "ORF-S17." Because the electroporation necessitated antibiotic selection, an empty vector plasmid was 
also electroportated into the S17 cell line and called "EMT-S17." Both lines were screened for uptake of the plasmid using puromycin selection. Effectively the EMT-S17 should model the original gene trap line, with one copy of Rps19 inactivated, and the ORF-S17 should serve as a control line. The second Rps19 model of haploinsufficiency has a gene trap insertion between exons three and four of the E14 parental cell line to generate the YHC074 gene trap line. The $R p / 5$ model was created by gene trap insertion between exon three and four of the Rp/5 gene in the parental TBV cell line to generate the D050 mutant line (Fig 3 and 4 of Chapter 3 ). 


\section{$\underline{\text { Results }}$}

In order to establish the gene trap cell lines as useful models of DBA, reduced expression of the gene products that have been targeted must be established. All three cell lines were found to contain the reporter gene mRNA by qRT-PCR as well as knockdown of the mRNA transcript for Rps19 or Rp/5 where appropriate (personal communication via Dr. Tracie Goldberg and Dr. Sharon Singh of the Feinstein Institute for Medical Research, data not shown). The Rps19 mES cells provided the most consistent and reproducible protein knockdown (Fig. S2). Our collaborators at the Feinstein Institute were able to show an initial protein knockdown in the Rp/5 samples (Fig. S3). 
A

YHC E14

S19 Mutant Control

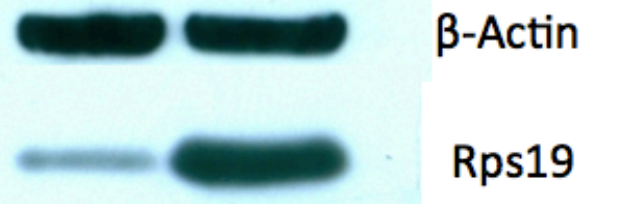

Approx. 80\% knockdown

B
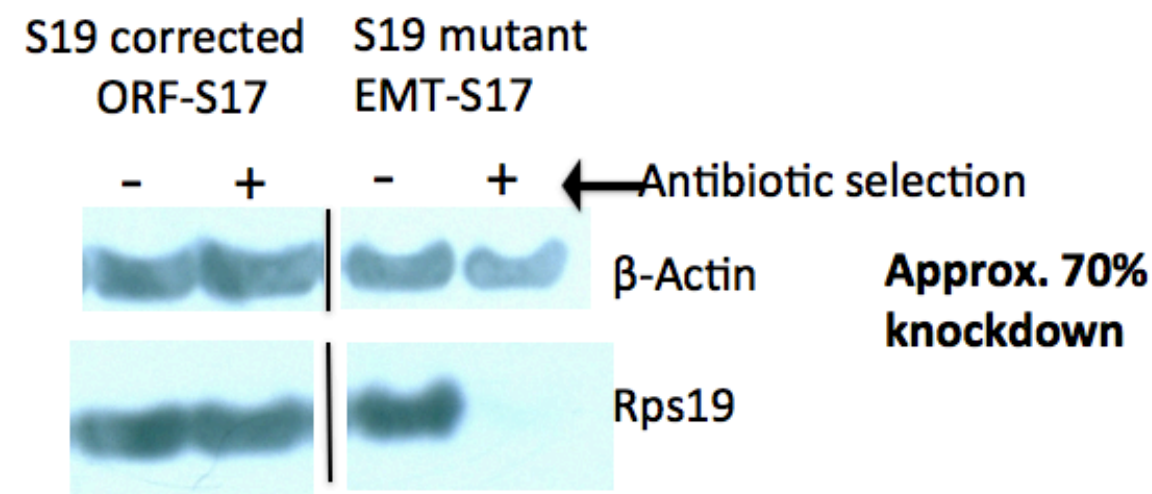

Fig S2: Protein knockdown of Rps19 in mES cells evidenced by immunoblotting.

Whole cell lysates were used for immunoblotting using antibodies raised against Rps19 and $\beta$-Actin. (A) YHC (Rps19 gene trap) and corresponding E14 parental control blotted for Rps19 and $\beta$-Actin. Band density was quantified by ImageJ software and showed an $\sim 80 \%$ knockdown of Rps 19 protein expression relative to the $\beta$-Actin loading control. (B) ORF-S17 and EMT-S17 mES gene trap samples grown with or without antibiotic selection to maintain the gene trap construct. The ORF-S17 is a corrected clone of the gene trap and therefore used as a control, while the EMT-S17 only has one functional copy of Rps19. 
The blot was quantified using Image $J$ and was determined to have approximately $30 \%$ knockdown of Rps19 protein expression relative to $\beta$-Actin loading control. 


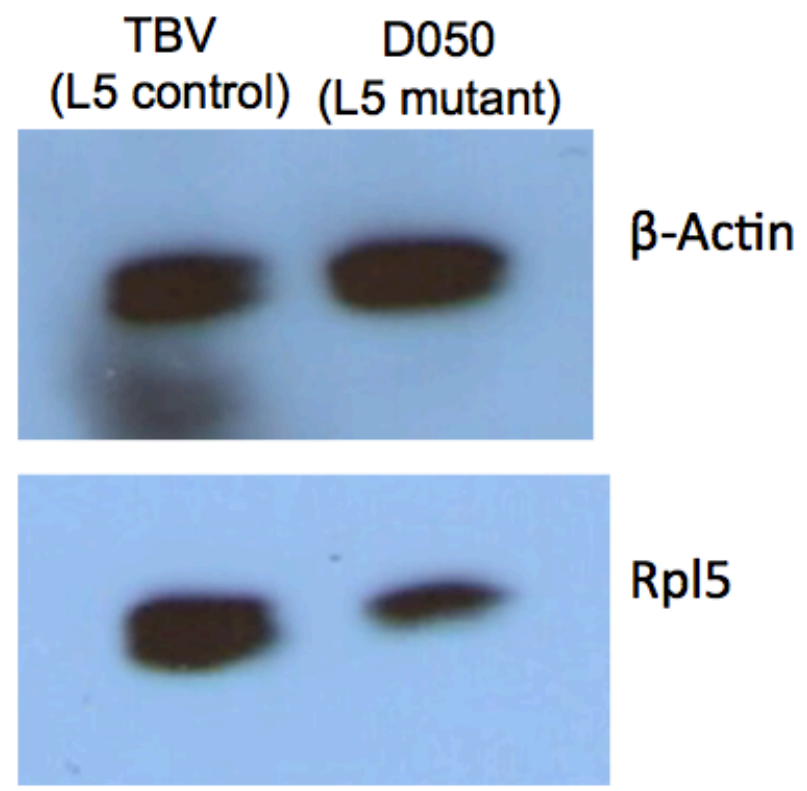

Fig S3: Protein knockdown of Rpl5 in mES gene trap samples.

D050 Rp/5 mutants and their corresponding YHC parental control mES whole cell lysates were Western blotted for Rpl5 and $\beta$-Actin expression by Dr. Tracie Goldberg. 
In addition mRNA and protein knockdown, characteristic processing defects in the maturation of rRNA as outlined in Chapter three should also be present. Total RNA was isolated from mES cells and used for Northern blotting as previously outlined (Flygare et al., 2007). In both DBA patients and cell culture models with haploinsufficiency for Rps19, there is a delay in the processing of $18 \mathrm{~S}$ rRNA that can be identified at the cleavage step of $21 \mathrm{~S}$ prerRNA into the more mature 18SE pre-rRNA when total RNA is Northern blotted using a probe called "18SE." This probe is complementary to the 3 ' end of the 18S rRNA and the 5' end of ITS1. This processing defect is evident as an increase in the ratio of the $21 \mathrm{~S}$ precursor relative to $18 \mathrm{SE}$. Figure S4 shows a representative Northern blot of the YHC Rps19 mutant cell line and its parental control, E14. The average ratio of $21 \mathrm{~S}$ to $18 \mathrm{SE}$ in four separate experiments in represented in the bar graph next to the blot. This increase in the 21S:18SE ratio represents an average 1.55 fold change when the mutant is normalized to the parental sample, which is on par with the inducible mouse model presented in supplemental section 2 . 


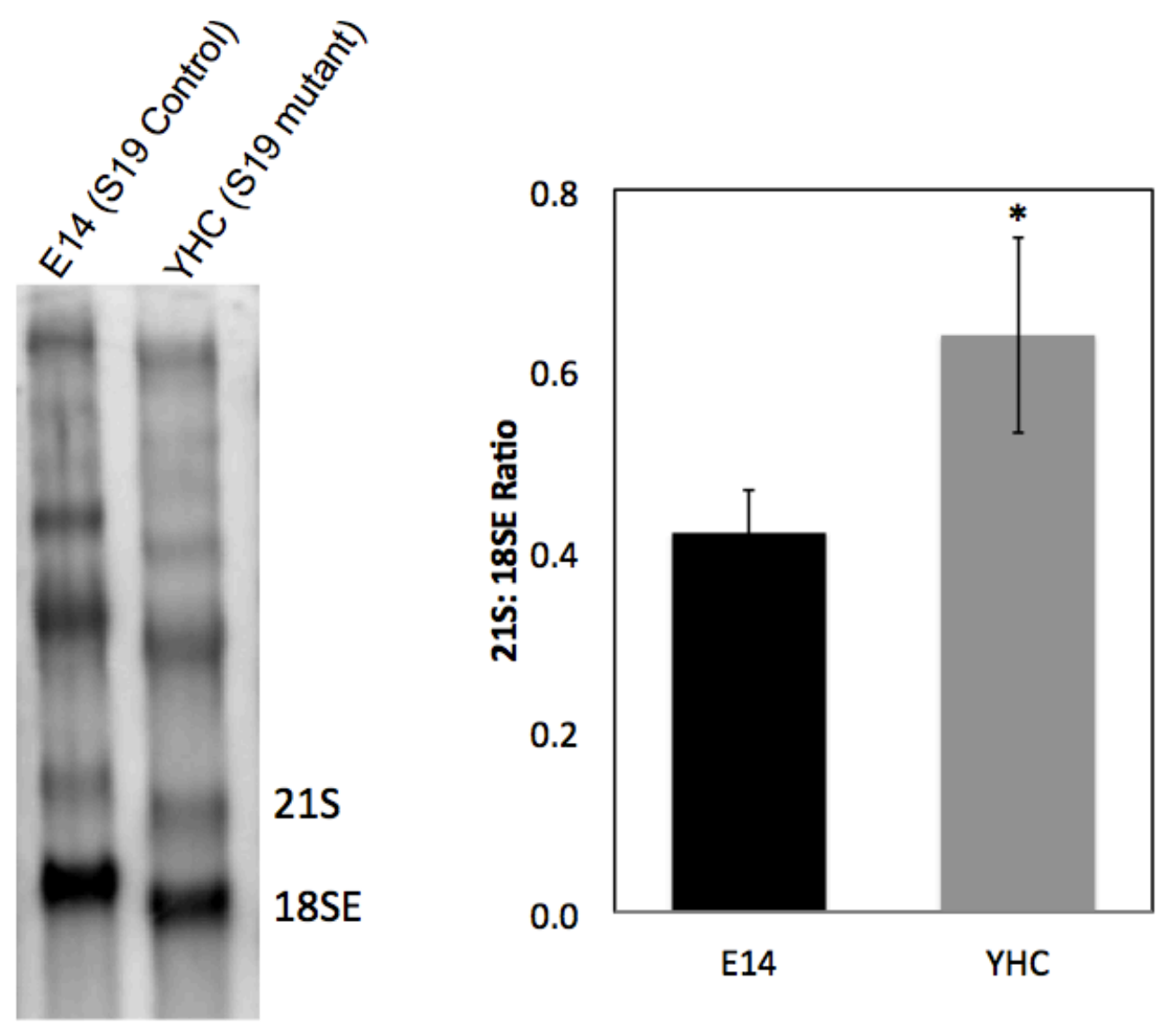

Fig S4: Mouse embryonic stem cells (mES) heterozygous for Rps19 display processing defects in pre-rRNA expected for Rps19 haploinsufficiency.

Representative Northern blot (left) and quantification of replicate data of total RNA harvested from mES cells with gene trap for Rps19 and parental controls. An increased ratio of $21 \mathrm{~S}$ to $18 \mathrm{SE}$ pre-rRNA is evident. This delay in the prerRNA processing was reproducible and statistically significant. (Bar graph represents the mean, with S.D. used for error bars, $n=4,{ }^{*} p<0.05$. As determined by an unpaired Student's t-test) 
To determine if the gene trap cell lines affected the production of ribosomal subunits we also analzyed polysome profiles from these cell lines. Figures S5 and S6 show representative polysome profiles from mES samples. 
A Interpreting Polysome Profiles
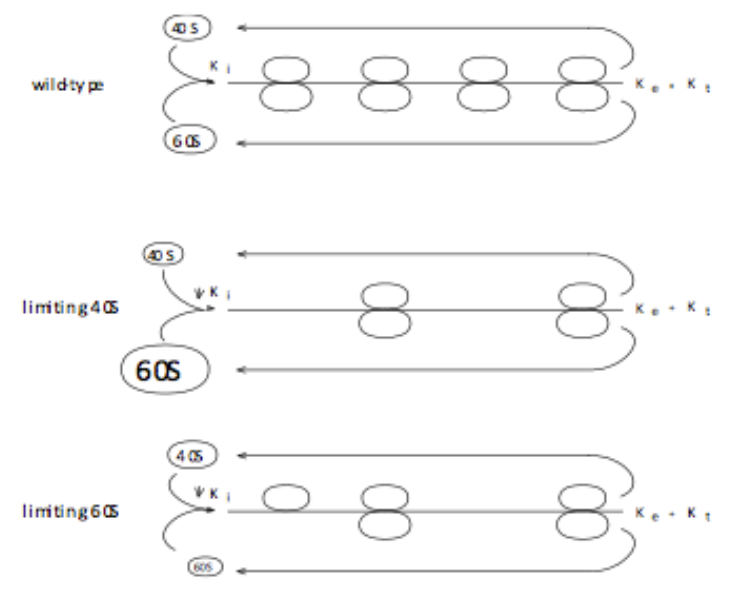

B

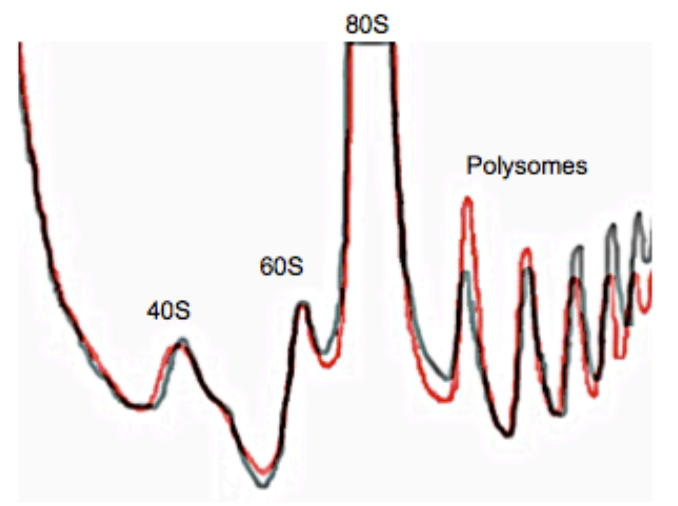

Fig. S5: mES samples with haploinsufficiency for $R p / 5$ show a decrease in polysome size.

(A) A schematic representing changes in polysome profiles depending on which ribosomal subunit is affected. Polysome profiling gives a general overview of the translational machinery in cells. The number of ribosomes translating a given mRNA at any time is dependent on the rates of initiation, elongation, and termination as shown in the top example in portion (A). Changes in levels of the $40 S$ and 605 subunits typically affect initiation giving smaller polysomes. The larger the number of ribosomes on a message the higher the initiation rate.

When the $40 \mathrm{~S}$ subunit becomes limiting, such as in models of Rps19 haploinsufficiecy represented in the middle schematic, there are fewer ribosomes attached to a given mRNA, causing a left shift in polysomes and a relative excess of free 605 subunits. This occurs because the $40 S$ subunit must bind to 
the mRNA first before a pre-initiation complex can prepared for subsequent 605 subunit binding. The bottom example, in which 605 subunits are limiting, as in Rp/5 haploinsufficiency, a 40S subunit may be attached to the mRNA, but has difficulty locating a 605 subunit capable of binding to it which leads to fewer $80 \mathrm{~S}$ initiation complexes formed and reduced initiation rates.

(B) This shows a representative polysome profile from $\mathrm{mES}$ samples with a gene trap targeting Rp/5. The parental cell line absorbance tracing $(\mathrm{YHC})$ is shown in black with the mutant (D050) absorbance tracing shown in red. Though the 60S subunit peak does not seem to be as severely affected as in the Rps19 models to follow, a modest defect appears in the polysome region and a slight leftward shift in polysomes indicating initiation rates may be affected. 
A

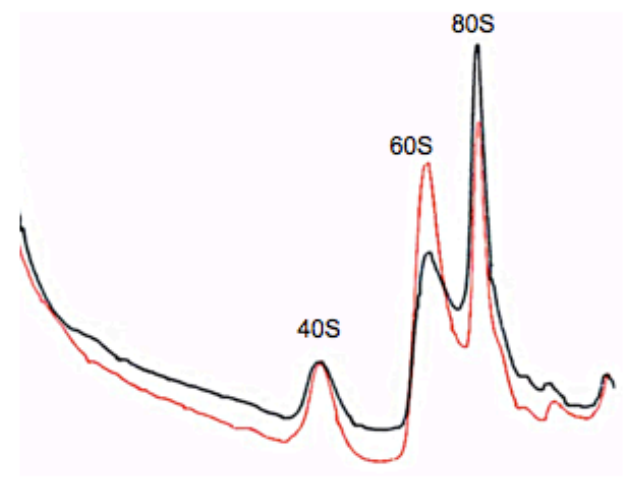

B

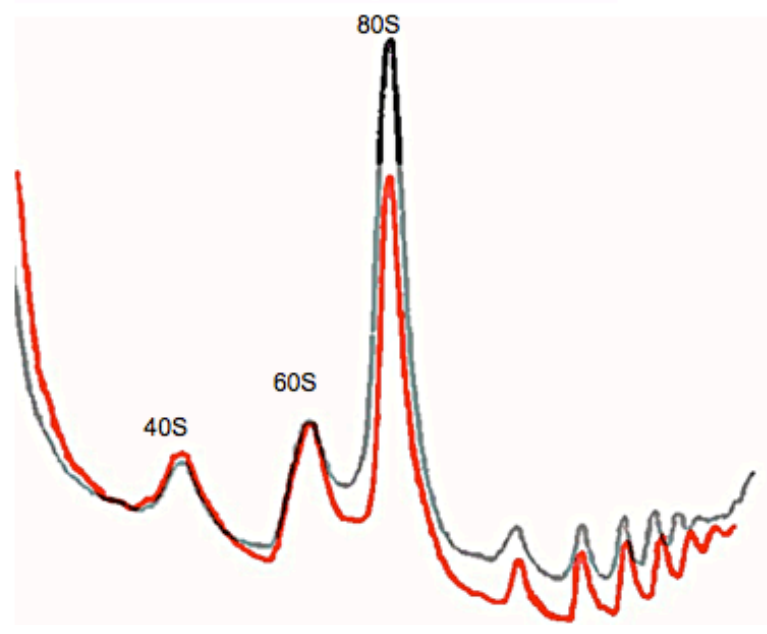

Fig. S6: mES polysome profiles in Rps19 genetrap.

Two mES cell lines with gene traps targeting Rps19 were treated with cyclohexamide and harvested for polysome profiling. (A) The parental E14 mES line generated the absorbance tracing shown in black and the mutant YHC absorbance tracing was overlaid in red. When normalized to the $40 \mathrm{~S}$ subunit, there is an appreciable relative decrease in the $80 \mathrm{~S}$ subunit. The $60 \mathrm{~S}$ subunit appears to be proportionally increased, but this is to be expected in the setting of abortive ribosomal assembly of $40 \mathrm{~S}$ subunits when Rps 19 is in limiting quantities. (B) A representative absorbance tracing of the ORF-S17 mES gene trap with corrected Rps19 protein expression is shown in black and the EMT-S17, mutant $\mathrm{mES}$ cell line is overlaid in red. The most appreciable defects in this system are 
found in both the $80 \mathrm{~S}$ subunit formation decrease and also as a trend toward smaller polysomes in the polysome region of the profile. 


\section{Discussion}

The protein knockdown of the ribosomal proteins targeted by the gene trap cell lines in addition to the ribosomal defects shown in polysome profiles, are suggestive of a specific subunit defect. At best, these data are far from concrete, but demonstration of ribosome synthesis deficiencies have never been as robust as those in human model systems. In addition to the ribosomal biology findings presented in the results section, collaborators at the Feinstein Institute made several important findings not presented as data in this dissertation.

They have been able to demonstrate defects in embryoid body formation as well as the ability to generate hematopoietic type colonies in all three cell lines, but the $R p / 5$ line uniquely generated smaller individual embryoid bodies. Preliminary data has shown a decreased ability for definitive erythropoiesis in all three cell lines, but the YHC cell line was able to maintain the efficiency of primitive erythropoiesis. There was also an increase in the p53 expression based on flow cytometry, but the initial experiments are not yet replicated. All defects tested were rescued by either p53 inactivation or cDNA re-expression of the gene targeted in the gene trap. Additionally, knockdown of Rp/11 expression in addition to the original genetrap defects increased all measureable defects. We hypothesize this is due to the role of Rpl11 in the MDM2-p53 pathway in cooperation with 5S rRNA and Rpl5. 


\section{Conclusions and Future Directions}

The mES model represented here is taking shape as a method to recapitulate and further investigate the molecular mechanisms leading to the block in maturation of erythroid precursors in DBA. There are several studies represented here and in the works of our collaborators that need to be replicated and statistically analyzed. Once these findings are determined to be reproducible they can be used to test potential therapies. It represents a model of haploinsufficiency that has been difficult to produce in whole animal models. As a tissue culture method, it offers a high throughput method to screen multiple drugs, or other therapies, and generate a specific dose response to a positively screened compound. Also, it has the specific advantage of analysis at the specific phases of hematopoietic differentiation of HSC that is the unifying feature of the constellation of features that make DBA a diagnostic and therapeutic challenge. If the defect in maturation of pre-erythroid cells can be reversed in this model, the potential validity is greatly increased. It certainly lends itself well to a pre-screening of potential therapies before the needless sacrifice of animals to potentially hazardous treatments. 


\title{
Section 2: Inducible Rps19 Depletion Mouse Model
}

\author{
$\underline{\text { Introduction }}$
}

This section represents some data used in the manuscript, Jaako et al in Blood 2011 (Jaako et al., 2011), though all figures used were newly adapted for this dissertation. This section outlines the validation of a potential mouse model for DBA. An inducible knockdown of the most common DBA gene, Rps19, was developed to circumvent several pitfalls of previous animal models. By allowing for normal expression of ribosomal protein genes during fetal development, the embryonic lethality shown with the homozygous deletion of Rps19 (Matsson et al., 2004) was avoided, and by adding one or two copies of an inducible shRNA to the genome of the animal theoretically creates an ability to express a broad range of Rps19 knockdown levels. Thus, this animal model is uniquely poised to display phenotypic manifestations of Rps19 knockdown in contrast to the heterozygous knockout mouse that showed very few changes in any of the originally measured parameters (Matsson et al., 2004). 


\section{$\underline{\text { Results }}$}

Embryonic fibroblasts (MEF) cells were harvested for growth in tissue culture as previously described (Jaako et al., 2011). As outlined in the methods section, the samples contained one (genotypes $\mathrm{B} /+$ and $\mathrm{D} /+$ ) or two (genotype $\mathrm{B} /$ B) copies of an inducible shRNA sequence targeting Rps19 mRNA labeled "B" or "D," under control of a tet-on promoter located in the collagen A1 locus.

MEF samples grown in culture in the presence of doxycycline were compared to untreated control cultures of the same genotype (labeled $+/+$ ). Total RNA was harvested from these cultures and used for Northern blot analysis to detect processing defects in the production of pre rRNA. Figure S7A details the portion of the pre-rRNA processing pathway examined using the "18SE" probe next to a representative Northern blot image. Both shRNA sequences increased the ratio of the immature $21 \mathrm{~S}$ precursor pre rRNA relative to the downstream cleavage product 18 SE pre-rRNA to a statistically significant level (Fig S7C). Mice given doxycycline in their drinking water for two weeks were sacrificed and their livers used for the same Northern blot analysis produced similar delays in the processing of pre-rRNA (Fig S7B). 


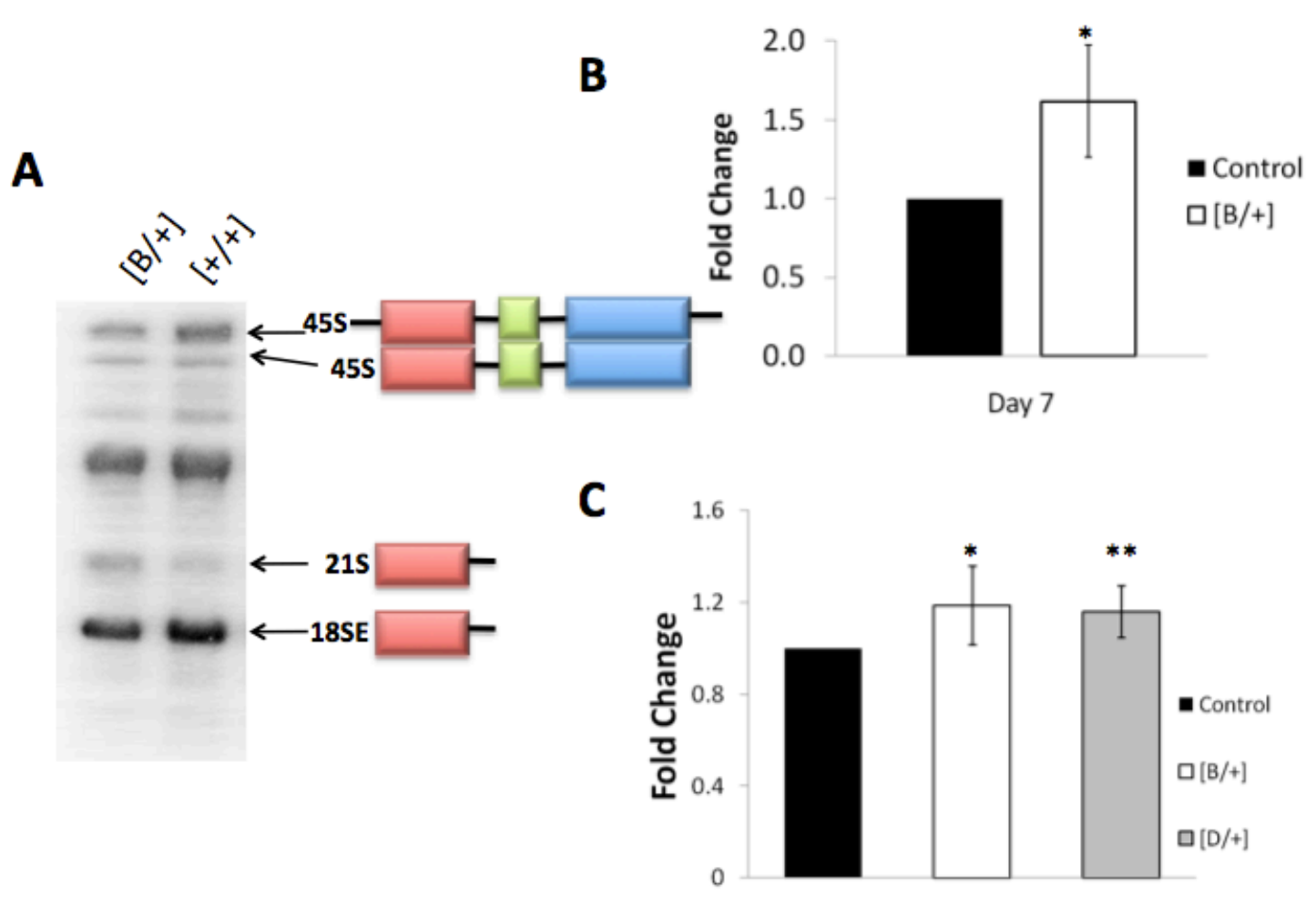

Fig S7: Pre-rRNA processing defects associate with inducible Rps19 shRNA expressing mouse model.

(A) Representative Northern blot of control $(+/+)$ or single copy B shRNA targeting Rps19 (B/+) MEF total RNA labeled with 18SE oligo on the left and the corresponding pre-rRNA species on the right. (B) Fold change in the ratio of 21S:18SE pre-rRNA species normalized to the ratio in the control MEF harvested after seven days of treatment with doxycycline $\left(n=4,{ }^{*} p<0.05\right)$.

(C) Fold changes in the ratio of $21 \mathrm{~S}: 18 \mathrm{SE}$ pre-rRNA species normalized to the ratio in the control. Total RNA from animals given doxycycline in their drinking water for fourteen days expressing $B(B /+)$ or $D(D /+)$ sequence shRNA to target Rps19 gene expression. 
MEF samples grown in culture, as well as liver and bone marrow samples harvested from animals treated with doxycycline for two weeks were used for polysome profiling. Figure S8 shows representative absorbance tracings from MEF, liver, and bone marrow samples. Peak intensity corresponds to the relative amount of polysomes in each peak.

MEF samples (Fig S8A) show a decrease in 40 S and 80 S subunits similar to profiles in bone marrow samples (Fig. S8C). Liver samples of the B/+ genotype show a relative increase in the free 605 pool as well as defects in $80 \mathrm{~S}$ formation (Fig S8B). Liver samples of the D/+ genotype show a more pronounced $40 \mathrm{~S}$ subunit defect as well as an $80 \mathrm{~S}$ biogenesis decrease and a slight decrease in the absorbance of the polysome region (Fig S8D). 


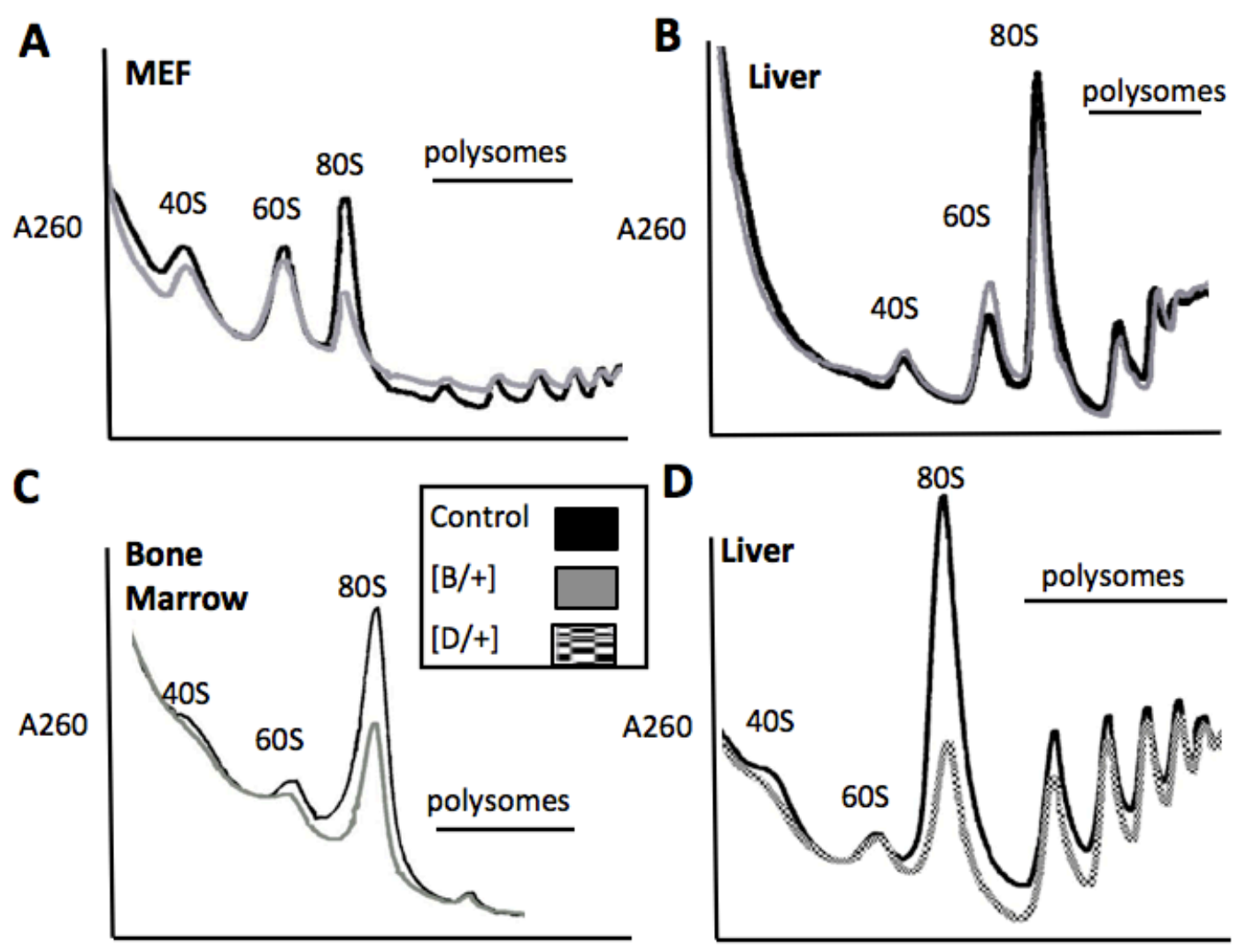

Fig S8: Polysome profiles of different cells and tissues from an inducible Rps19 shRNA mouse model.

$\begin{array}{ll}\text { (A) MEF control and }(B /+) & \text { (B) Liver control and }(B /+) \text { (C) Bone marrow control }\end{array}$ and $(B /+)$ and $(D)$ Liver control and $(D /+)$ representative absorbance tracings of cells harvested in cycloheximide and separated by size by ultracentrifugation on sucrose gradients. 
Printed absorbance tracings were trimmed at the baseline of each peak and the weight of the different peaks measured. The absorbance peak can be thought of as an integral such that the area under the curve (weight) is proportional to the number of polysomes in that peak. Liver polysome profile samples provided the most robust examples of small subunit deficiency in comparison to other tissue types sampled in these experiments, and peaks were weighed using this method. Peak weight is shown as a percentage of the $40 \mathrm{~S}$ subunit weight normalized to the 40 S subunit in Fig S9. 


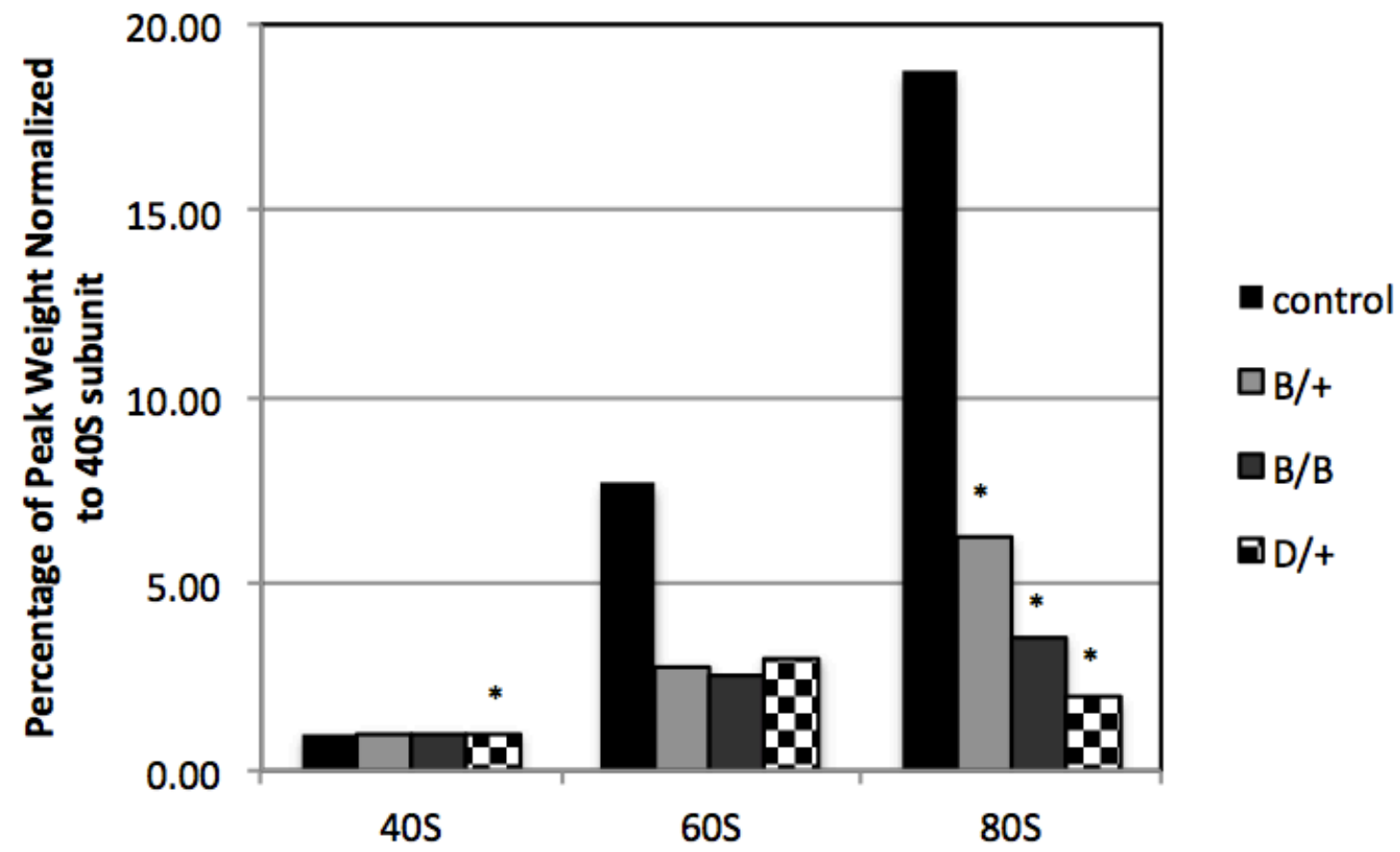

Fig S9: Peak weights of liver rRNA species separated on sucrose gradients and identified by polysome profiling.

Ribosomal RNA species were identified in absorbance tracings, and peaks cut out individually from the tracing and weighed. The bar graph represents each peak as a percentage of total profile weight normalized to the $40 \mathrm{~S}$ subunit $(n=6$, ${ }^{*} p<0.05$ as determined by an unpaired Student's t-test) 


\section{Discussion}

Multiple publications have shown a delay in the processing of pre-rRNA from the polycistronic $45 S$ precursor that generates the mature $18 S, 5.8 S$, and $28 \mathrm{~S}$ rRNA species in both patient samples and animal models (Flygare et al., 2007; Jaako et al., 2011; Robledo et al., 2008). This model showed similar increases in the ratio of 21S: 18SE pre-rRNA in both MEF (Fig. S7A and S7B) and liver (Fig. S7C) samples further validating this ratio as a method for measuring functional consequences of Rps19 deficiency.

Polysome profile analyses were similarly supportive of a specific small subunit defect (Fig. S7). The top left panel shows a representative diagram of MEF samples normalized to the $60 \mathrm{~S}$ subunit absorbance peak to highlight a corresponding decrease in the absorbance maximum for $40 \mathrm{~S}$ subunits as well as $80 \mathrm{~S}$ subunits, which is what would be predicted with a limiting quantity of $40 \mathrm{~S}$ subunits. This pattern was recapitulated in the liver samples with one inducible copy of the D shRNA shown in the bottom right panel. Normalizing the liver samples with one copy of the B shRNA sequence to the 40 S subunit also highlights a relative increase in the 605 subunit and decrease in the 805 subunit and polysome region shown in the top right panel. At the time of submission of this dissertation, I am unaware of any other work that has shown polysome profiles generated from bone marrow samples. The bottom left panel shows near absence of the 405 subunit as well as a decrease in the 805 peak absorbance. This data is less than ideal, but given the bone marrow failure of the animals depleted of Rps19, the starting material for this experiment was of limiting 
quantities. In liver samples each peak weight as a percentage of the total profile weight was graphed in Fig S8. The shRNA sequence "D" was shown to have a greater effect on Rps19 gene expression (data not shown) and a statistically significant decrease in $40 \mathrm{~S}$ subunit weight. The $80 \mathrm{~S}$ subunits of each of the Rps19 knockdown liver sample genotypes were shown to decrease, further supporting the functional lack of $40 \mathrm{~S}$ subunits due to a decrease in Rps19.

Though data was not shown in the manuscript, the mouse model central to these studies recapitulated many of the hematopoietic defects pathognomonic of DBA in addition to the ribosomal defects outlined above. These animals exhibit a macrocytic anemia, with additional defects in other blood lineages, which is not uncommon in the heterogeneous presentation of DBA (Farrar and Dahl, 2011). These animals also display symptoms of bone marrow failure over time based on competitive transplantation assay, which is a hallmark of DBA pathophysiology. The phenotype could be rescued by ectopic expression of Rps 19 or loss of p53, cementing the specificity of the model (Jaako et al., 2011). 


\section{Conclusions and Future Directions}

The molecular signatures highlighted by this work were previously published (Jaako et al., 2011). Without careful validation of the molecular pathophysiology characteristic of DBA, the specificity of an animal model is in question. The pre-rRNA processing and polysome profile defects link the bone marrow failure shown in the animal model to specific defects in Rps19. Now that this model has been validated, it has enormous potential in drug discovery and design, as well as more detailed inspection of the diverse biological pathways involved in DBA pathophysiology. 


\section{APPENDIX II}

\section{Abbreviations}

$\underline{\mathrm{CGH}}$ - comparative genomic hybridization

DBA- Diamond Blackfan Anemia

DC- Dyskeratosis Congenita

DMEM- dublbecco's modified eagle media

DNA- deoxyribonucleic acid

DTT- dithiothreitol

EB- embryoid body

Ex- exon

FBS - fetal bovine serum

FA- Fanconi Anemia

FFME - formaldehyde, formamide,MOPS, ethidium

$\underline{\text { FISH }}$ - flow in-situ hybridization

GMCSF- granulocyte macrophage colony stimulating factor

$\underline{\text { HRP- }}$ - horse radish peroxidase

IBMFS- inherited bone marrow failure syndrome

$\underline{\text { IMM- inner mitochondrial membrane }}$

$\underline{\text { IMS- intermembrane space }}$

ITS- internal transcribed sequence

$\underline{\mathrm{IDH}}$ - isocitrate dehydrogenase

LTR- long terminal repeat 
MM- mitochondrial matrix

MEF- mouse embryonic fibroblasts

mES- mouse embryonic stem cells

mLIF- mouse leukemia inhibitory factor

MMRRC- mutant mouse regional resource center

mRNA - messenger RNA

$\underline{\mathrm{MTG}}$ - monotioglycerol

OMM- outer mitochondrial membrane

ORF- open reading frame

$\underline{P / S}-$ penicillin and streptomycin solution

$\underline{\mathrm{PDH}}$ - pyruvate dehydrogenase

RNA- ribonucleic acid

ROS- reactive oxygen species

$\underline{\mathrm{RPL}}$ - ribosomal protein of the large subunit

RPS- ribosomal protein of the small subunit

rRNA- ribosomal RNA

SA- splice acceptor

$\underline{\mathrm{SCN}-}$ severe congenital neutropenia

SD- splice donor

SDS- Shwachman Diamond Syndrome

SnoRNP-small nucleolar ribonuclear protein

SOD2- super oxide dismutase 2 (mitochondrial)

TOMM- transfer channel of the outer mitochondrial membrane 
tRNA- transfer RNA

TSA- trichostatin A

VDAC- voltage dependent anion channel

\section{Gene Abbreviations}

elF6- eukaryotic initiation factor 6

RPS19 (Rps19)- small ribosomal subunit protein 19

$\underline{R P L 5(R p / 5)}$ - large ribosomal subunit protein 5

$\underline{R P L 11(R p / 11)}$ - large ribosomal subunit protein 11

$\underline{S B D S}$ - shwachman bodian diamond syndrome protein

SIRT3- $N A D(+)$ dependent deacetylase sirtuin 3, mitochondrial 


\section{CURRICULUM VITAE}

NAME: Adrianna Lee Henson

ADDRESS: 2223 Sycamore Ave \#2

Louisville, KY 40206

PHONE: $\quad$ 828-773-6789

EMAIL: alhens03@louisville.edu

DOB: $\quad$ Boone, NC - May 18, 1983

EDUCATION \& TRAINING:

University of Louisville School of Medicine: Louisville, KY

$\mathrm{MD} / \mathrm{PhD}$ Student with a graduate studies focus in Biochemistry and Molecular Biology

August 2006- present

Wake Forest University: Winston Salem, NC

BS in Chemistry with a minor in Biology

August 2001-May 2005

Watauga High School: Boone NC

HONORS AND AWARDS:

MD/PhD program University of Louisville

One of two fully funded students entering in 2006

Third place in poster competition at Midwest Blood Symposium 2011

Wake Forest University

George F. Hankins Scholarship

Zachary T. Smith Scholarship

William K. Stamey Scholarship

Robert C. Byrd Scholarship

Recipient of Distinguished Service Key: Alpha Phi Omega 2005

Dean's list Fall 2001 and Fall 2002-Spring 2005 


\section{RESEARCH EXPERIENCE:}

University of Louisville

Summer Research Scholars Program 2007

Department of Biochemistry

Molecular Targets Program

Summer Research Scholars Program 2006

Molecular Targets Program

Wake Forest University School of Medicine

Plastic and Reconstructive Surgery Dept. May 2005- May2006

Research Tech II for Dr. Louis Argenta and Dr. Lisa David

Department of Biochemistry January 2005-May 2005

Student Research Assistant for Dr. Suzi Torti

Department of Cancer Biology October 2001-May 2004

Student Research Assistant for Dr. Andrew Thorburn

\section{TEACHING/MENTORING EXPERIENCE:}

University of Louisville Mentees

Julie Ann Smith

Max Wattenberg

Millicent Fugate

Morgan Partin

Dr. Aisha Zaidi

PUBLICATIONS, PRESENTATIONS, POSTERS:

* indicates presenter ${ }^{* *}$ co-first authorship

Manuscript Under Revision

Goldberg $T^{* *}$, Singh $S^{* *}$, Henson A, Solaimanzadeh J,Blanc L, A, Nihrane A, Ellis SR, Lipton JM, Liu J. A defect in primitive erythropoiesis is associated with a G2/M cell cycle arrest in Rpl5 mutant murine embryonic stem cell model of Diamond Blackfan Anemia.

Manuscript Under Revision

Fisher R, et al. Identification of Novel Pathogenic DBA Mutation.

Manuscript under revision

Henson A, Moore J, Alard P, Wattenberg M, Liu J, Ellis S. Mitochondrial function is disrupted in yeast and human cellular models of Shwachman Diamond syndrome. 
Poster

Henson $\mathbf{A}^{*}$, Fisher R, Ellis S. Identification of a Novel Gene Affected in the Inherited Bone Marrow Failure Syndrome, Diamond Blackfan Anemia.

National MD/PhD Student Conference: Keystone, CO, 2012.

Journal Article

Sezgin $G^{* *}$, Henson $A^{* *}$, Singh S, Nihrane A, Singh S, Wattenberg M, Alard P, Ellis S, Liu J. Impaired Growth, Hematopoietic Colony Formation, and Ribosome Maturation in Human Cells Depleted of ShwachmanDiamond Syndrome Protein SBDS. Pediatr Blood Cancer. 2012 Sep 19.

Presentation

Liu J, Goldberg T, Singh S, Henson A, Solaimanzadeh J, Ellis S, Lipton J. Ontogenetic and mechanistic differences in two murine es models of diamond Blackfan anemia: infantile versus fetal anemia. Daniella Maria Arturi Foundation Meeting: New York, NY, 2012.

Journal Article

Jaako P, Flygare J, Olsson K, Quere R, Ehinger M, Henson A, Ellis S, Schambach A, Baum C, Richter J, Larsson J, Bryder D, Karlsson S. Mice with ribosomal protein $\mathrm{S} 19$ deficiency develop bone marrow failure and symptoms like patients with Diamond-Blackfan anemia. Blood. 2011 Dec 1;118(23):6087-96.

Poster

Henson A $^{*}$, Moore J, Liu J, Ellis S. Mitochondrial dysfunction as a potential source of reactive oxygen species in cellular models of shwachman-diamond syndrome. American Society of Hematology: San Diego, CA, 2011.

Journal Article

Henson AL, Ellis SR. Finding a diamond in the (mouse is) rough. Blood. 2010 Oct 14 ; 116(15): 2623-5.

Journal Article

Donninger H, Allen N, Henson A, Pogue J, Williams A, Gordon L, Kassler $S$, Dunwell T, Latif F, Clark GJ. Salvador protein is a tumor suppressor effector of RASSF1A with hippo pathway-independent functions. J Biol Chem. 2011 May 27;286(21): 18483-91.

Presentation

Sezgin G, Nihrane A, Henson A, Wattenberg M, Ellis S, Liu J. Impaired ribosome maturation in human cells depleted of shwachman-diamond syndrome protein SBDS. Shwachman-diamond syndrome congress: New York, NY 2011. 
Poster

Sezgin G, Nihrane A, Henson A*, Wattenberg M, Ellis S, Liu J. Impaired ribosome maturation in human cells depleted of shwachman-diamond syndrome protein SBDS. American Society of Pediatric Hematology Oncology: Baltimore, MD, 2011.

Poster

Henson A $^{*}$, Jaako P, Karlsson S, Ellis S. Ribosomal defects found in tissues of diamond blackfan anemia mouse model. Midwest Blood Club: Cincinnati, OH, 2011.

Presentation

Goldberg T, Singh S, Henson A, Nihrane A, Lipton J, Ellis S, Liu J. Unique primitive erythropoiesis defect in rpl5-deficient murine embryonic stem cell model of diamond Blackfan anemia. American Society of Hematology: 2010, Orlando, FL, 2010.

Poster

Sezgin G, Nihrane A, Henson A*, Wattenberg M, Ellis S, Liu J. Impaired ribosome maturation in human cells depleted of shwachman-diamond syndrome protein SBDS. American Society of Hematology: Orlando, FL, 2010.

Poster

Wattenberg M, Henson A, Moore J, Ellis S. Cellular models of shwachman diamond syndrome. Research! Louisville: Louisville, KY, 2010.

Poster

Goldberg T, Henson A*, Singh S, Nihrane A, Lipton J, Ellis S, Liu J. Specific hematopoietic and erythroid differentiation defects in mouse embryonic stem (es) cells with abortive ribosome assembly. American Society of Hematology: New Orleans, LA, 2009.

Poster

Pavesi, E. Henson A*. Nucleolar stress signaling through MDM2 and p53 stabilization as the basis for the pro-apoptotic phenotype in DBA. James Graham Brown Cancer Retreat: Louisville, KY, 2008.

Poster

Henson A, Clark G. Salvador is a novel human tumor suppressor that links RASSF1A to Bax. Research! Louisville: Louisville, KY, 2007. 
Poster

Henson A, Campain J, Eaton J. Cytotoxic effects of atherosclerotic gruel and a potential strategy for pharmacologic treatment of atherosclerosis.

Research! Louisville: Louisville, KY, 2006.

Presentation

Park C, Sanger C, Argenta A, Simpson J, Henson A, Green H, Voignier D, Gordon S, David L. "Outcome analysis of the treatment of severe positional plagiocephaly with passive helmet therapy." Annual meeting of the American Society of Plastic Surgeons. Oct 2006. San Francisco, CA.

Journal Article

Thomas LR, Henson A, Reed JC, Salsbury FR, Thorburn A. Direct binding of Fas-associated death domain (FADD) to the tumor necrosis factor-related apoptosis-inducing ligand receptor DR5 is regulated by the death effector domain of FADD. J Biol Chem. 2004 Jul 30;279(31): 32780-5.

\section{EXTRACURRICULAR AND LEADERSHIP ACTIVITIES:}

University of Louisville

University of Louisville Student Chapter of American Medical Women's Associations

Fall 2007-Spring 2008

Offices: Treasurer

Pathology Course Reform Committee

Spring 2008

Publicity Committee for the Health Care Classic

Fall 2007

Wake Forest University

$\mathrm{Phi} \mathrm{Mu}$

Spring 2003-Spring 2005

Offices: Philanthropy Chair

University Orchestra

Fall 2001-Fall 2004

WFU Club Swimming

Fall 2001-Fall 2002

Wesley Foundation

Fall 2001-Fall 2002 


\section{SERVICE ACTIVITIES:}

University of Louisville

Student Leadership Core Group

Fall 2007-Spring 2008

Offices: Volunteer Coordinator for the Class of 2010

Walking Works Mentor

Fall 2011-current

Wake Forest University

Alpha Phi Omega: Service Fraternity

Spring 2002-Spring 2005

Offices: President, Treasurer, Service Chair, and Sergeant of Arms

PROFESSIONAL MEMBERSHIPS:

Member American Medical Women's Associations (AMWA)

Fall 2007-Spring 2008

Member American Medical Student Association (AMSA)

Fall 2006-Current

Member American Medical Association (AMA)

Fall 2006-Current

Member Kentucky Medical Association (KMA)

Fall 2006- Current

Member Southern Medical Association (SMA)

Fall 2006-Current

Member Phi Delta Epsilon

Spring 2008-Current

HOBBIES AND OUTSIDE INTERESTS:

My family

Running

Swimming

Reading

Hiking

Knitting

Gardening 